\title{
Incidence of debris discs around FGK stars in the solar neighbourhood ${ }^{\star}$
}

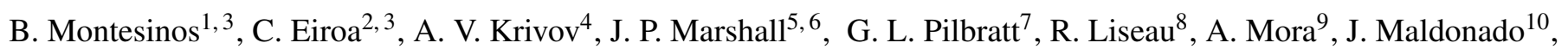
S. Wolf ${ }^{11}$, S. Ertel $^{12}$, A. Bayo ${ }^{13,14}$, J.-C. Augereau ${ }^{15,16}$, A. M. Heras ${ }^{7}$, M. Fridlund ${ }^{8,17}$, W. C. Danchi ${ }^{18}$, E. Solano ${ }^{1,19}$, F. Kirchschlager ${ }^{11}$, C. del Burgo ${ }^{20}$, and D. Montes ${ }^{21}$

1 Departmento de Astrofísica, Centro de Astrobiología (CAB, CSIC-INTA), ESAC Campus, Camino Bajo del Castillo s/n, 28692 Villanueva de la Cañada, Madrid, Spain e-mail: benjamin.montesinos@cab.inta-csic.es

2 Universidad Autónoma de Madrid, Dpto. Física Teórica, Módulo 15, Facultad de Ciencias, Campus de Cantoblanco, 28049 Madrid, Spain

3 Unidad Asociada CAB-UAM

4 Astrophysikalisches Institut und Universitätssternwarte, Friedrich-Schiller-Universität, Schillergäßchen 2-3, 07745 Jena, Germany

5 School of Physics, UNSW Australia, NSW 2052 Sydney, Australia

6 Australian Centre for Astrobiology, UNSW Australia, NSW 2052 Sydney, Australia

7 ESA, Directorate of Science, Scientific Support Office, European Space Research and Technology Centre (ESTEC/SCI-S), Keplerlaan 1, 2201 AZ Noordwijk, The Netherlands

8 Department of Earth and Space Sciences, Chalmers University of Technology, Onsala Space Observatory, 43992 Onsala, Sweden

9 ESA-ESAC Gaia SOC, PO Box 78, 28691 Villanueva de la Cañada, Madrid, Spain

10 INAF, Osservatorio Astronomico di Palermo, Piazza Parlamento 1, 90134 Palermo, Italy

11 Institute of Theoretical Physics and Astrophysics, Christian-Albrechts University Kiel, Leibnizstr. 15, 24118 Kiel, Germany

12 Steward Observatory, Department of Astronomy, University of Arizona, 933 North Cherry Avenue, Tucson, AZ 85721, USA

13 Instituto de Física y Astronomía, Facultad de Ciencias, Universidad de Valparaíso, Av. Gran Bretaña 1111, 5030 Casilla, Valparaíso, Chile

14 ICM Nucleus on Protoplanetary Disks, Universidad de Valparaíso, Av. Gran Bretaña 1111, 2360102 Valparaíso, Chile

15 Univ. Grenoble Alpes, IPAG, 38000 Grenoble, France

16 CNRS, IPAG, 38000 Grenoble, France

17 Leiden Observatory, University of Leiden, PO Box 9513, 2300 RA Leiden, The Netherlands

18 NASA Goddard Space Flight Center, Exoplanets and Stellar Astrophysics, Code 667, Greenbelt, MD 20771, USA

19 Spanish Virtual Observatory, Centro de Astrobiología (CAB, CSIC-INTA), ESAC Campus, Camino Bajo del Castillo s/n, 28692 Villanueva de la Cañada, Madrid, Spain

${ }^{20}$ Instituto Nacional de Astrofísica, Óptica y Electrónica, Luis Enrique Erro 1, Santa María de Tonantzintla, Puebla, México

21 Departamento de Astrofísica, Facultad de Ciencias Físicas, Universidad Complutense de Madrid, 28040 Madrid, Spain

Received 17 February 2016 / Accepted 18 May 2016

\section{ABSTRACT}

Context. Debris discs are a consequence of the planet formation process and constitute the fingerprints of planetesimal systems. Their counterparts in the solar system are the asteroid and Edgeworth-Kuiper belts.

Aims. The aim of this paper is to provide robust numbers for the incidence of debris discs around FGK stars in the solar neighbourhood. Methods. The full sample of 177 FGK stars with $d \leq 20$ pc proposed for the DUst around NEarby Stars (DUNES) survey is presented. Herschel/PACS observations at 100 and $160 \mu \mathrm{m}$ were obtained, and were complemented in some cases with data at $70 \mu \mathrm{m}$ and at 250,350 , and $500 \mu \mathrm{m}$ SPIRE photometry. The 123 objects observed by the DUNES collaboration were presented in a previous paper. The remaining 54 stars, shared with the Disc Emission via a Bias-free Reconnaissance in IR and Sub-mm (DEBRIS) consortium and observed by them, and the combined full sample are studied in this paper. The incidence of debris discs per spectral type is analysed and put into context together with other parameters of the sample, like metallicity, rotation and activity, and age.

Results. The subsample of 105 stars with $d \leq 15$ pc containing $23 \mathrm{~F}, 33 \mathrm{G}$, and $49 \mathrm{~K}$ stars is complete for F stars, almost complete for $\mathrm{G}$ stars, and contains a substantial number of $\mathrm{K}$ stars from which we draw solid conclusions on objects of this spectral type. The incidence rates of debris discs per spectral type are $0.26_{-0.14}^{+0.21}$ ( 6 objects with excesses out of $23 \mathrm{~F}$ stars), $0.21_{-0.11}^{+0.17}$ ( 7 out of $33 \mathrm{G}$ stars), and $0.20_{-0.09}^{+0.14}$ (10 out of $49 \mathrm{~K}$ stars); the fraction for all three spectral types together is $0.22_{-0.07}^{+0.08}$ (23 out of 105 stars). The uncertainties correspond to a $95 \%$ confidence level. The medians of the upper limits of $L_{\text {dust }} / L_{*}$ for each spectral type are $7.8 \times 10^{-7}(\mathrm{~F}), 1.4 \times 10^{-6}(\mathrm{G})$, and $2.2 \times 10^{-6}(\mathrm{~K})$; the lowest values are around $4.0 \times 10^{-7}$. The incidence of debris discs is similar for active (young) and inactive (old) stars. The fractional luminosity tends to drop with increasing age, as expected from collisional erosion of the debris belts.

Key words. stars: late-type - circumstellar matter - protoplanetary disks - infrared: stars

\footnotetext{
* Herschel is an ESA space observatory with science instruments provided by European-led Principal Investigator consortia and with important participation from NASA.
} 


\section{Introduction}

Star and planet formation are linked by the presence of a circumstellar disc built via angular momentum conservation of the original molecular cloud undergoing gravitational collapse (e.g. Armitage 2015). These primordial discs, formed by gas and small dust particles, evolve with the star during the pre-main sequence (PMS) phase. They experience gas dispersal and grain growth and are transformed from gas-dominated protoplanetary discs to tenuous, gas-poor, dusty discs, known as "debris discs". These discs consist of $\mu \mathrm{m}$-sized particles with short lifetimes and need to be constantly replenished. Hence, they are secondgeneration discs, thought to be produced by the constant attrition - due to collisional cascades - of a population of planetesimals (e.g. Wyatt 2008; Krivov 2010).

Primordial discs around young stars are the birth sites of planets and provide the initial conditions for planet formation, the raw material, and the systems' architectures. Some of these young discs have built the $\sim 3400$ exo-solar planets - known to date - distributed in several hundreds of multiple planetary systems that have been found in the last $20 \mathrm{yr}$, mostly around main-sequence (MS) stars ${ }^{1}$. The observed planetary systems as a whole, i.e. planets and/or debris discs, present a wide variety of architectures (e.g. Marshall et al. 2014; Moro-Martín et al. 2015; Wittenmyer \& Marshall 2015) that, in conjunction with the host stars, determine the fate of the systems once the stars abandon the MS phase towards later phases of stellar evolution (e.g. Mustill et al. 2014).

A large effort has been devoted to the field of debris discs, with fundamental contributions from space astronomy in the mid- and far-infrared $(\lambda \gtrsim 10 \mu \mathrm{m})$ since the pioneering discovery of an infrared excess in the spectral energy distribution (SED) of Vega provided by the InfraRed Astronomical Satellite (IRAS, Aumann et al. 1984). An enormous number of references could be provided to cover the vast area of debris discs, their properties, morphologies, and relationships with the presence of planets. Concerning the topics addressed in this paper, the Infrared Space Observatory (ISO, 1995-1998) enlarged the sample of stars observed, studying for the first time the incidence rate of debris discs around MS AFGK stars (Habing et al. 2001) and the time dependency of Vega-like excesses (Decin et al. 2003). The Spitzer observatory (cryogenic mission 2003-2009) provided large leaps forward both qualitatively and quantitatively, showing that $\sim 16 \%$ of solar-type FGK stars have dusty discs (Trilling et al. 2008). The sensitivity of these three missions to debris disc detection was limited by their aperture sizes.

The ESA Herschel space observatory (2009-2013) (Pilbratt et al. 2010) has been fundamental in extending the picture thanks to its $3.5 \mathrm{~m}$ aperture and its imaging photometers PACS (Poglitsch et al. 2010) and SPIRE (Griffin et al. 2010), which provide an increased sensitivity to debris discs (see Fig. 1 in Eiroa et al. 2013), a wider wavelength coverage, and the ability to spatially resolve many of the discs. An account of results of the pre-Herschel era can be found in the review by Wyatt (2008), whereas a much more comprehensive view, including some of the Herschel results, is given by Matthews et al. (2014).

Eiroa et al. (2013, hereafter E13), presented the results obtained from the Herschel open time key programme (OTKP) DUst around NEarby Stars (DUNES) ${ }^{2,3}$. This programme aimed to detect Edgeworth-Kuiper belt (EKB) analogues around nearby

\footnotetext{
1 See http://exoplanetarchive.ipac.caltech.edu/ for updated numbers of exoplanets and multi-planet systems.

2 http://www.mpia-hd.mpg.de/DUNES/

3 http://sdc.cab.inta-csic.es/dunes/
}

Table 1. Summary of spectral types in the DUNES_DU and DUNES_DB samples.

\begin{tabular}{lcccc}
\hline \hline Sample & F & G & K & Total \\
\hline DUNES_DU & 27 & 52 & 54 & 133 \\
$\leq 15$ pc DUNES_DU subsample & 4 & 19 & 43 & 66 \\
$\leq 20$ pc DUNES_DU subsample & 19 & 50 & 54 & 123 \\
\hline DUNES_DB & 51 & 24 & 6 & 81 \\
$\leq 15$ pc DUNES_DB subsample & 19 & 14 & 6 & 39 \\
$\leq 20$ pc DUNES_DB subsample & 32 & 16 & 6 & 54 \\
\hline
\end{tabular}

Notes. Nomenclature of the samples: DUNES_DU contains the stars of the DUNES sample observed by the DUNES team; DUNES_DB contains the stars of the DUNES sample observed by the DEBRIS team.

solar-type stars. The incidence rate of debris discs in a $d \leq 20 \mathrm{pc}$ subsample was $20 \pm 2 \%$. However, the sample of FGK stars observed and analysed in that paper was not complete (see Sect. 2.2 for details); therefore, it was necessary to analyse data on a complete or near-complete sample in order to avoid biased conclusions.

This paper analyses the full sample of solar-type (FGK) stars of the DUNES programme located at $d \leq 20 \mathrm{pc}$, as described in the original DUNES proposal, i.e. the subsample studied in E13 plus the stars shared with and observed within the OTKP Disc Emission via a Bias-free Reconnaissance in IR and Sub-mm (DEBRIS, Matthews et al. 2010). The scientific background, context, and rationale of the present work are the same as those presented in E13; they were described in detail in Sects. 1 and 2 of that paper and the information is not repeated here. Special attention is paid to the DUNES subsample of stars with $d \leq 15 \mathrm{pc}$, which is complete for F stars, almost complete for $\mathrm{G}$ stars, and has a substantial number of $\mathrm{K}$ stars, making the conclusions concerning the incidence rate of debris discs in the corner of our Galaxy fairly robust.

The paper is organized as follows. In Sect. 2, the sample of stars is described; the reasons why the observations of the full DUNES sample were split between the DUNES and DEBRIS teams are explained; the completeness of the sample is discussed, and comprehensive information on the optical and nearIR photometry of the shared sample used to build the spectral energy distributions is also given. In Sect. 3, the observations and data reduction are described. The results are presented in Sect. 4. The analysis of the full DUNES sample is done in Sect. 5 and a summary of the main conclusions is presented in Sect. 6 .

\section{Stellar sample}

\subsection{Selection criteria}

The stellar sample analysed in this work is the merger of the sample studied in E13 (hereafter called DUNES_DU) and a subsample of FGK stars observed by the DEBRIS team (hereafter identified as DUNES_DB). The full DUNES sample is composed of the merger of DUNES_DU and DUNES_DB. We concentrate our analysis on the stars with $d \leq 20 \mathrm{pc}$, and when addressing the incidence rates of debris discs, we pay special attention to the subset within 15 pc. Below, we give details of the DUNES_DU and DUNES_DB subsamples.

DUNES and DEBRIS were two complementary Herschel programmes with different observing strategies and different approaches to the selection criteria for their samples. Given the 
overlapping scientific interests of both projects, the Herschel Time Allocation Committee suggested splitting the samples in the most convenient and efficient way, keeping the philosophy and observing strategy of the corresponding science cases. Both teams were granted the same amount of observing time $(140 \mathrm{~h})$.

As described in E13, the original DUNES stellar sample, from which the final sample was built, was chosen from the HIPPARCOS catalogue (VizieR online catalogue I/239/hip_main, Perryman et al. 1997) following the only criterion of selecting MS stars - luminosity class V-IV/V - closer than $25 \mathrm{pc}$, without any bias concerning any property of the objects. The restriction to building the final sample was that the stellar photospheric emission could be detected by PACS at $100 \mu \mathrm{m}$ with a $S / N \geq 5$, i.e. the expected $100 \mu \mathrm{m}$ photospheric flux should be significantly higher than the expected background as estimated by the Herschel HSPOT tool at that wavelength. Two stars, namely $\tau$ Cet (HIP 8102, G8 V) and $\epsilon$ Eri (HIP 16537, K2 V), although fulfilling all the selection criteria described above, do not belong to the DUNES sample because they were included in the Guaranteed Time Key Programme "Stellar Disk Evolution" (PI: G. Olofsson).

Taking into account the amount of observing time allocated and the complementarity with DEBRIS, the sample observed by the DUNES team was restricted to main-sequence FGK solar-type stars located at distances shorter than 20 pc. In addition, FGK stars between 20 and 25 pc hosting exoplanets (three stars to date, one F-type and two G-type) and previously known debris discs, mainly from the Spitzer space telescope (six stars, all F-type) were also included. Thus, the DUNES_DU sample ${ }^{4}$ is composed of 133 stars, 27 of which are F-type, 52 G-type, and $54 \mathrm{~K}$-type stars. The 20 pc subsample is formed of 123 stars, 19 F-type, 50 G-type, and 54 K-type ${ }^{5}$.

The OTKP DEBRIS was defined as a volume-limited study of A through M stars selected from the "UNS" survey (Phillips et al. 2010), observing each star to a uniform depth, i.e. DEBRIS was a flux-limited survey. In order to optimize the results according to the DUNES and DEBRIS scientific goals, the complementary nature of the two surveys was achieved by dividing the common stars of both original samples, considering whether the stellar photosphere could be detected with the DEBRIS uniform integration time and assigning those stars to DEBRIS. In this way, the DUNES observational requirement of detecting the stellar photosphere was satisfied. The few common A- and M-type stars in both surveys were also assigned to DEBRIS.

The net result of this exercise was that 106 stars observed by DEBRIS satisfy the DUNES photospheric detection condition and are, therefore, shared targets. Specifically, this sample comprises 83 FGK stars: 51 F-type, 24 G-type, and 8 K-type (the remaining stars are of spectral type $\mathrm{A}$ and $\mathrm{M}$ ). We note that spectral types listed in the HIPPARCos catalogue are used to give these numbers; we see below that two $\mathrm{K}$ stars had to be excluded owing to an incorrect spectral type classification. Since the assignment to one of the teams was made on the basis of

\footnotetext{
4 This subsample was formally called the "DUNES sample" in E13, but it is actually only the subset of the full DUNES sample that was observed within the DUNES observing time.

5 In E13, the sample of stars within $20 \mathrm{pc}$ was composed of $124 \mathrm{ob}-$ jects because the selection of the initial sample was done according to the original ESA 1997 release of the HIPPARCos catalogue; in that release the parallax of HIP 36439 was $50.25 \pm 0.81$ mas. However, in the revision by van Leeuwen (2007) the parallax is $49.41 \pm 0.36$ mas, putting this object beyond 20 pc. Here we exclude this star from the DUNES_DU 20 pc sample, and consider only 123 stars in that subset.
}

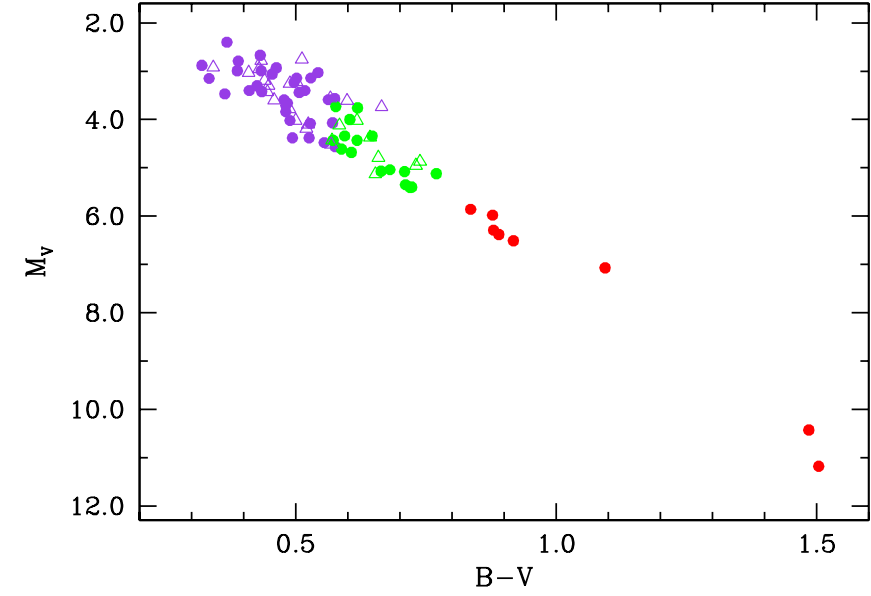

Fig. 1. HR diagram for the FGK stars of the DUNES sample observed by the DEBRIS team (DUNES_DB). The colour codes following the HIPPARCos spectral types are F stars (violet), G stars (green), and $\mathrm{K}$ stars (red). Solid circles represent FGK stars closer than 20 pc; empty triangles are FGK stars further away. The two red dots at the bottom right of the diagram are two stars classified as K5 in the HIPPARcos catalogue, but their colours correspond to M stars. See text for details.

both the DUNES and DEBRIS original samples, the number of shared targets located closer than $20 \mathrm{pc}$, i.e. the revised DUNES distance, is lower, namely 56 FGK stars: 32 F-type, 16 G-type, and 8 K-type stars.

In Fig. 1 we show the HR diagram $M_{V}-(B-V)$ for the sample of FGK stars of the DUNES sample observed during the DEBRIS observing time. Solid circles represent FGK stars closer than $20 \mathrm{pc}$ - i.e. the subsample analysed in this paper whereas empty triangles have been used to plot FGK stars further away, not included in the study presented here. Two stars, namely HIP 84140 and HIP 91768 - the two red dots at the bottom right of the diagram - are both classified as K5 in the HIPPARCOS catalogue, but their $(B-V)$ colours clearly correspond to that of an $\sim$ M3 star (the spectral type listed in SIMBAD for both objects), and so they have been moved out of the FGK star sample. We identify the subsample of 81 FGK stars as DUNES_DB in this paper, out of which 54 are closer than $20 \mathrm{pc}$. Table 1 summarizes the spectral type distribution of the DUNES_DU and DUNES_DB samples, taking into account these two misclassifications.

Table C. 1 provides some basic information of the FGK stars within $d \leq 20 \mathrm{pc}$ of the DUNES_DB sample (the corresponding information for the DUNES_DU sample can be found in Table 2 of E13). HIPPARCOS spectral types are given in the table. In order to check the consistency of these spectral types we have explored VizieR using the DUNES discovery tool ${ }^{6}$ (see Appendix A in E13). Results of this exploration are summarized in Col. 5 which gives the spectral type range of each star taken into account SIMBAD, Gray et al. (2003, 2006), Wright et al. (2003), and the compilation made by Skiff (2009). The typical spectral type range is $2-3$ subtypes. The parallaxes are taken from van Leeuwen (2007, VizieR online catalogue I/311). Parallax errors are typically less than 1 mas. Only one star, HIP 46509, has an error larger than 2 mas, this object being a spectroscopic binary (see Table C.3). The multiplicity status of the DUNES_DB sample is addressed in Sect. 2.4.

6 http://sdc.cab.inta-csic.es/dunes/searchform.jsp 


\subsection{Completeness}

The main constraint affecting the completeness of the sample is the observational restriction that the photosphere had to be detected with a $S / N \geq 5$ at $100 \mu \mathrm{m}$. It is important to know the impact of this restriction in order to assess the robustness of the results concerning the frequency of the incidence of debris discs in the solar neighbourhood.

In Fig. 2 the cumulative numbers of stars in the merged DUNES_DU and DUNES_DB $d \leq 15$-pc and $d \leq 20$-pc samples - normalized to the total number of objects in each one - have been plotted against stellar mass as red and blue histograms, respectively. The masses have been assigned to each star by linear interpolation of the values given for FGK MS stars by Pecaut \& Mamajek $(2013)^{7}$ using $(B-V)$ as the independent variable. The expected mass spectrum for the solar neighbourhood according to a Salpeter law (Salpeter 1955) with exponent -2.35 has been normalized to the total cumulative number of each sample and plotted in green. It is clear that the sample with $d \leq 20$ pc shows an underabundace of stars below $\sim 1.2 M_{\odot}$, whereas the behaviour of the sample with $d \leq 15 \mathrm{pc}$ approximates that of the Salpeter law, but still runs slightly below at low masses. Similar results are obtained using other approaches for the mass spectrum, e.g. that by Kroupa (2001). In grey, the cumulative distribution for the FGK stars with $d \leq 15 \mathrm{pc}$ from the HIPPARCos catalogue has also been included, showing a much closer agreement with the Salpeter law.

Since the DUNES sample was drawn from the HIPPARCos catalogue, it is important to assess the problem of its completeness. In Sect. 2.2 of Turon et al. (1992) it can be seen that the HIPPARCos Input Catalogue was only complete down to $V=7.3$ for stars cooler than G5. For $d=15 \mathrm{pc}$, this corresponds to a spectral type of $\sim \mathrm{K} 2$. In addition, not all the stars in the Input Catalogue were observed (see Table 1 of Turon et al. 1992): for the magnitude bin $V: 7-8$ the efficiency of the survey was $93 \%$; therefore, even applying a safety margin of 0.3 mag, we would have completeness only up to spectral type K1. In summary, down to $15 \mathrm{pc}$, HIPPARcos is complete for F and G stars, but not for K stars.

To quantify the departure of the full DUNES $d \leq 15$-pc sample from the parent HIPPARCos set, we compared the number of stars of each spectral type in our sample with the corresponding numbers in the HIPPARCos catalogue. In Fig. 3, cumulative histograms of the numbers of F, G, and $\mathrm{K}$ stars (luminosity classes V and IV-V) in the HIPPARCos catalogue (red), and in the DUNES sample (black), both at $d \leq 15$, are plotted against distance. The total numbers of stars are $23 \mathrm{~F}, 42 \mathrm{G}, 89 \mathrm{~K}$ in HIPPARCos against $23 \mathrm{~F}, 33 \mathrm{G}, 49 \mathrm{~K}$ in the DUNES sample, i.e. the observational constraint imposed in the final selection of the targets keeps all the F stars from HIPPARCos, but discards $9 \mathrm{G}$ and $40 \mathrm{~K}$ stars. Therefore, a correction on the fraction of excesses extracted from the DUNES sample would have to be applied - in particular for K stars - in order to give results referring to a wider sample. We come back to this point in Sect. 4 .

\subsection{Stellar parameters of the DUNES_DB sample}

Table C.2 gives some parameters of the DUNES_DB objects with $d<20 \mathrm{pc}$, namely the effective temperature, gravity, and metallicity; the flag in Col. 5 indicates whether the determination of these parameters was spectroscopic or photometric. The

\footnotetext{
7 See also "Stellar Color/Teff Table" under section "Stars" in http: //www.pas.rochester.edu/ emamajek/
}

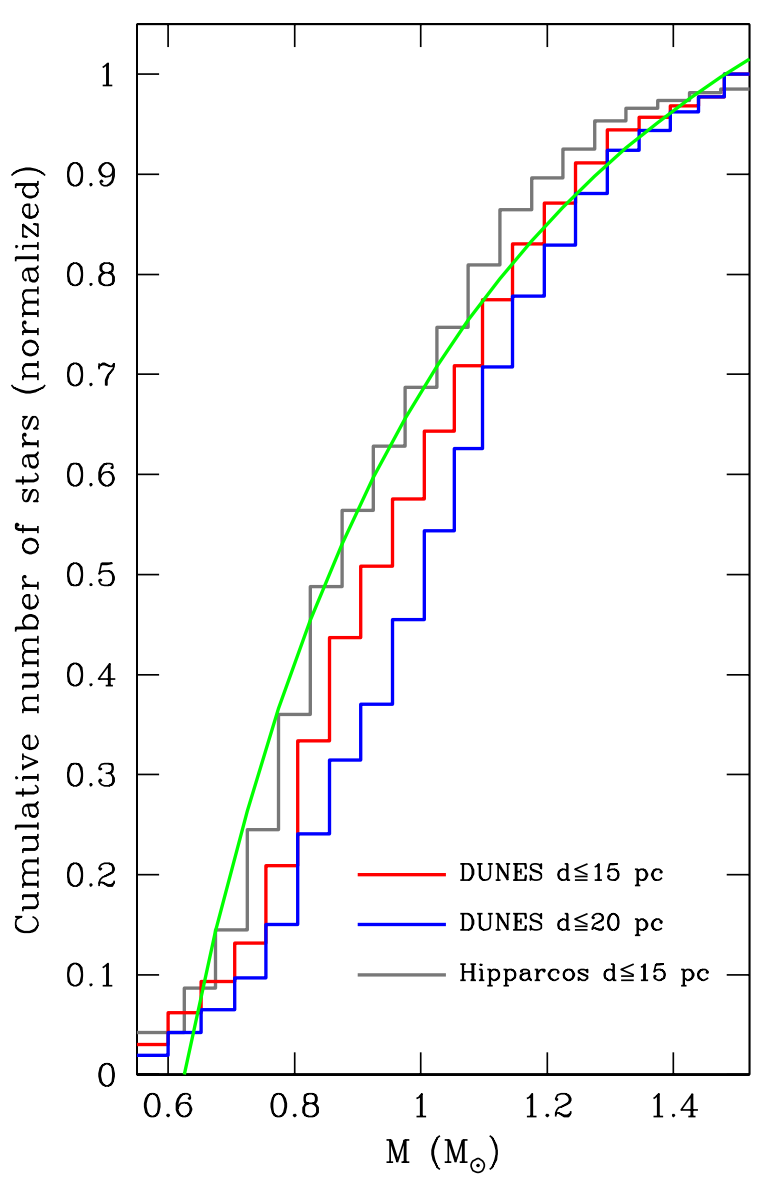

Fig. 2. Cumulative distributions (normalized) for the DUNES samples with $d \leq 15 \mathrm{pc}$ (105 stars, red) and $d \leq 20 \mathrm{pc}$ (177 stars, blue) and the FGK stars in the HIPPARCos catalogue with $d \leq 15$ pc (154 stars, grey), all plotted against stellar mass. A Salpeter law with exponent -2.35 has been normalized to the distributions. See text for details.

stellar luminosity and the activity indicator $\log R_{\mathrm{HK}}^{\prime}$, are also given. References for $T_{\text {eff }}, \log g,[\mathrm{Fe} / \mathrm{H}]$, and $\log R_{\mathrm{HK}}^{\prime}$ are at the bottom of the table. The luminosity was computed using Eq. (9) in Torres (2010), with the values of the $V$-magnitude and colour index $(B-V)$ taken from the HIPPARCos catalogue, and the bolometric corrections (BC) from Flower (1996); $(B-V)$ was used as the independent variable to obtain $\mathrm{BC}$.

The rotation periods, $P_{\text {rot }}$, have been estimated using the strong correlation between $\log R_{\mathrm{HK}}^{\prime}$ and the Rossby number, defined as $\mathrm{Ro}=P_{\text {rot }} / \tau_{\mathrm{c}}$, where $\tau_{\mathrm{c}}$ is the convective turnover time, which is a function of the spectral type (colour; Noyes et al. 1984; Montesinos et al. 2001; Mamajek \& Hillenbrand 2008). For six objects, the estimation of $P_{\text {rot }}$ was not feasible because the calibrations do not hold for the values of either $\log R_{\mathrm{HK}}^{\prime}$ or $B-V$; in that case, lower limits of the rotation period, based on the values of $v \sin i$, are given. The stellar ages have been computed according to gyrochronology $\left(t_{\text {Gyro }}\right)$ and using the chromospheric emission as a proxy for the age $\left(t_{\mathrm{HK}}\right)$. In Appendix A we give details of the estimation of the rotation periods and ages.

\subsection{Multiplicity}

Table C.3 lists the multiplicity properties of the objects that are known to be binaries. The importance of this information cannot be underestimated, especially when the spectral types of the components are close - and hence $\Delta V$ is small - and the binary 


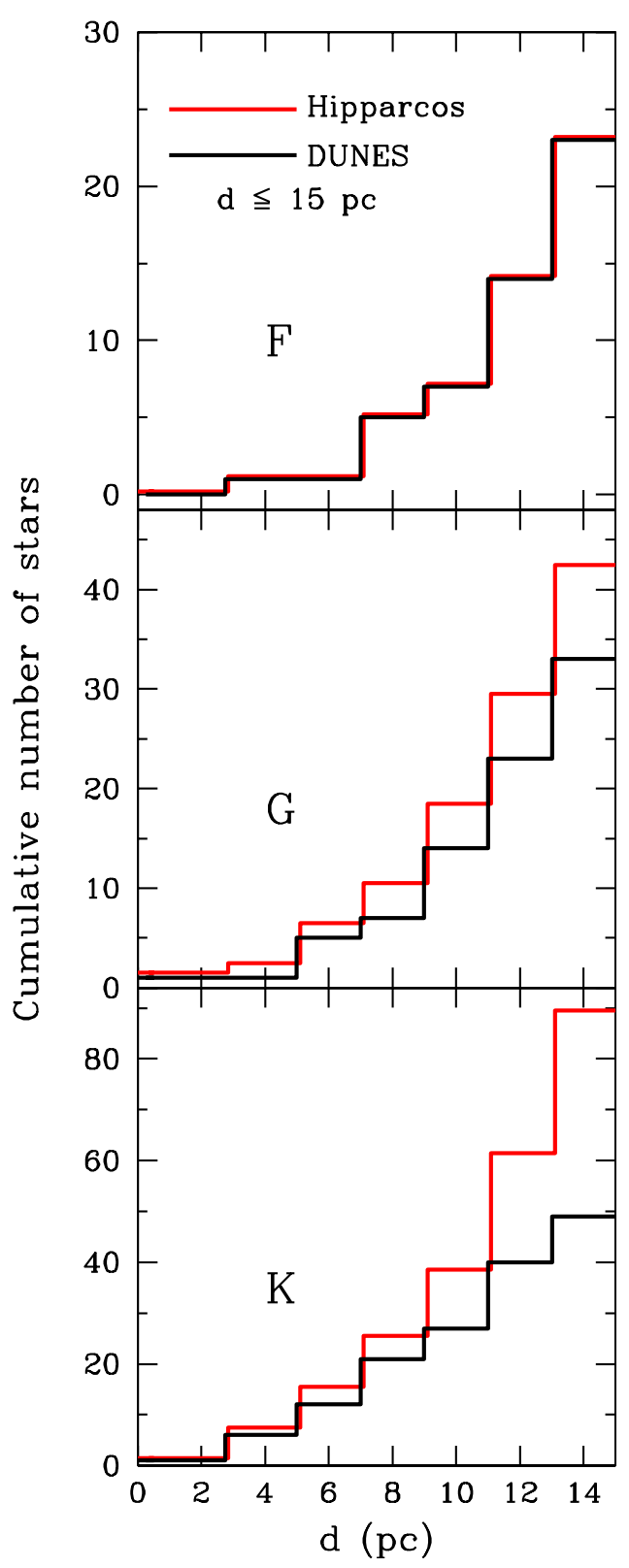

Fig. 3. Cumulative number of $F, G$, and $\mathrm{K}$ stars (luminosity classes $\mathrm{V}$ and IV-I) in the HIPPARCos catalogue (red) and in the DUNES sample (black), both at $d \leq 15 \mathrm{pc}$.

is spectroscopic or its components have not been resolved individually during a particular spectroscopic or photometric observation from which a given parameter is derived. In many cases parameters are assigned as representative of the whole system, without reference in the literature to which component they refer to. This also has an impact on the calculation of the corresponding model photospheres that are used (after normalization to the optical and near-IR photometry) as baselines to detect potential excesses at the Herschel wavelengths. Concerning the data given in Table C.3, this has to be taken into account for HIP 7751 $(\Delta V=0.16)$, HIP 44248 (1.83), HIP 61941 (0.04), HIP 64241 (0.68), HIP 72659 (2.19), HIP 73695 (0.87), and HIP 75312 (0.37), and for those stars classified as "spectroscopic binaries" for which no information on their components is available. The proper motions of the components have been extracted from the "HIPPARCos and Tycho catalogues (Double and Multiples: Component solutions)" (VizieR online catalogue I/239/h_dm_com).
The bottom part of Table C. 3 gives information about the stars of the DUNES_DB sample with $d \leq 20 \mathrm{pc}$ that to date have confirmed exoplanets. The mass and the length of the semimajor axis of the orbit for each planet are given.

\subsection{Photometry}

Tables C.4-C.7 give the optical, near-IR, AKARI, WISE, IRAS, and Spitzer MIPS magnitudes and fluxes of the DUNES_DB stars with $d \leq 20 \mathrm{pc}$, and the corresponding references. This photometry has been used to build and trace the spectral energy distributions (SEDs) of the stars, which are - along with PHOENIX/Gaia models (Brott \& Hauschildt 2005) - the tools used to predict the fluxes at the far-IR wavelengths in order to determine whether or not the observed fluxes at those wavelengths are indicative of a significant excess. Appendix C in E13 gives details on the photospheric models and the normalization procedure to the observed photometry. In addition to the photometry, Spitzer/IRS spectra of the targets, built by members of the DUNES consortium from original data from the Spitzer archive, are included in the SEDs, although they have not been used in the process of normalization of the photospheric models.

\section{Herschel observations of the DUNES_DB sources and data reduction}

\subsection{PACS observations}

As described by Matthews et al. (2010), DEBRIS is a fluxlimited survey where each target was observed to a uniform depth $\left(1.2 \mathrm{mJy} \mathrm{beam}^{-1}\right.$ at $\left.100 \mu \mathrm{m}\right)$. The observations of the DUNES_DB sources were performed by the DEBRIS team with the ESA Herschel Space Observatory using the instrument PACS. Further details can be found in the paper by Matthews et al. (2010). The main parameters of the observational set-up used by DEBRIS are compatible with those used by DUNES and are described in E13.

\subsection{Data reduction}

Data for each source comprise a pair of mini scan-map observations taken with the PACS 100/160 waveband combination. DEBRIS and DUNES observations both followed the same pattern for mini scan-map observations (see PACS Observer's Manual, Chap. 5); minor differences in the scan leg lengths ( $8^{\prime}$ for DEBRIS, $10^{\prime}$ for DUNES) lead to slightly different coverage across the resulting map areas for the two surveys, in addition to the different map depths. These differences are immaterial to the analysis presented here. The Herschel/PACS observations were reduced in the Herschel interactive processing environment (HIPE, Ott 2010). For the analysis presented here we used HIPE version 10 and PACS calibration version 45 . Reduction was carried out following the same scheme as presented in E13. Table C.8 shows a $\log$ of the observations with details of the OBSIDs and the on-source integration times.

The individual PACS scans of each target were high-pass filtered to remove large-scale background structure, using highpass filter radii of 20 frames at $100 \mu \mathrm{m}$ and 25 frames at $160 \mu \mathrm{m}$, suppressing structures larger than $82^{\prime \prime}$ and $102^{\prime \prime}$, respectively, in the final images. For the filtering process, a region of $30^{\prime \prime}$ radius around the source position in the map along with regions where the pixel brightness exceeded a threshold defined as twice the standard deviation of the non-zero flux elements in the map (determined from the level 2 pipeline reduced product) were masked 
from inclusion in the high-pass filter calculation. Regions external to the central $30^{\prime \prime}$ that exceeded the threshold were tagged as sources to be avoided in the background estimation phase of the flux extraction process. Deglitching was carried out using the spatial deglitching task in median mode and a threshold of $10 \sigma$. For the image reconstruction, a drop size (pixfrac parameter) of 1.0 was used. Once reduced, the two individual PACS scans in each waveband were mosaicked to reduce sky noise and suppress $1 / f$ striping effects from the scanning. Final image scales were $1^{\prime \prime}$ per pixel at $100 \mu \mathrm{m}$ and $2^{\prime \prime}$ per pixel at $160 \mu \mathrm{m}$, compared to native instrument pixel sizes of $3.2^{\prime \prime}$ for $100 \mu \mathrm{m}$, and $6.4^{\prime \prime}$ for $160 \mu \mathrm{m}$.

\section{Results}

The extraction of PACS photometry of the sources analysed in this paper follows identical procedures to those in E13. Section 6.1 .1 of that paper contains a very detailed description of the procedures used to carry out the flux extraction. In summary, flux densities were measured using aperture photometry. If the image was consistent with a point source, then an aperture appropriate to maximise the signal-to-noise was adopted (i.e. $5^{\prime \prime}$ at $100 \mu \mathrm{m}$ and $8^{\prime \prime}$ at $160 \mu \mathrm{m}$; see E13). Regarding extended sources, we refer the reader to Sect. 7.2.3 of E13 where the criteria are described for assessing whether the $3 \sigma$ flux contours in the 100 and $160 \mu \mathrm{m}$ images denote extended emission.

The background and rms scatter were estimated from the mean and standard deviation of ten square sky apertures placed at random around the image. The sky apertures were sized to have roughly the same area as the source aperture. The locations of the sky apertures were chosen such that they did not overlap with the source aperture or with any background sources (to avoid contamination), and lay within $60^{\prime \prime}$ of the source position (to avoid higher noise regions at the edges of the image).

Measured flux densities in each PACS waveband were corrected for the finite aperture size using the tabulated encircled energy fractions given in Balog et al. (2014), but have not been colour corrected $^{8}$. A point-source calibration uncertainty of $5 \%$ was assumed for all three PACS bands. We note that aperture corrections valid for point sources have also been applied to the case of extended sources. This is obviously an approach, but it should be valid as long as the aperture size is larger than the extended source. This criterion has widely been used without any apparent inconsistency in other works, e.g. Wyatt et al. (2012) for 61 Vir, Duchêne et al. (2014) for $\eta$ Crv, and Roberge et al. (2013) for 49 Cet.

Table C. 9 lists the PACS 100 and $160 \mu \mathrm{m}$ photometry and $1 \sigma$ uncertainties for the sources of the DUNES_DB sample with $d \leq 20 \mathrm{pc}$, identified as the far-IR counterparts of the optical stars. Uncertainties include both the statistical and systematic errors added in quadrature; the uncertainty in the calibration is $5 \%$ in both bands (Balog et al. 2014). Quantities without errors in the PACS160 column correspond to $3 \sigma$ upper limits. In the columns adjacent to the PACS photometry, the photospheric predictions, $S_{v}(\lambda)$, with the corresponding uncertainties are given. The fluxes $S_{v}(\lambda)$ are extracted from a Rayleigh-Jeans extrapolation of the fluxes at $40 \mu \mathrm{m}$ from the PHOENIX/Gaia normalized models. The uncertainties in the individual photospheric fluxes were estimated by computing the total $\sigma$ of the normalization in logarithmic units; in that calculation, the observed flux at each wavelength involved in the normalization process was

8 See PACS Technical Notes PICC-ME-TN-038 and
PICC-ME-TN-044.

compared with its corresponding predicted flux. The normalized model $\log S_{v}(\lambda)$ was permitted to move up and down a quantity $\pm \sigma$. That value of $\sigma$ was then translated into individual - linear - uncertainties of the fluxes, $\sigma\left(S_{v}(\lambda)\right)$, at the relevant Herschel wavelengths. The typical uncertainty in the photospheric predictions derived from a change of $\pm 50 \mathrm{~K}$ in $T_{\text {eff }}$ for a model with $6000 \mathrm{~K}$ is $\sim 0.9 \%$ of the predicted flux density. It does not have noticeable effects in any of the calculations.

We consider that an object has an infrared excess at any of the PACS wavelengths when the significance, calculated as

$\chi_{\lambda}=\frac{\operatorname{PACS}_{v}(\lambda)-S_{v}(\lambda)}{\left(\sigma\left(\operatorname{PACS}_{v}(\lambda)\right)^{2}+\sigma\left(S_{v}(\lambda)\right)^{2}\right)^{1 / 2}}$

is larger than 3.0, although in some cases the complexity of the fields makes it difficult to ascertain whether an apparent clear excess based solely on the value of $\chi_{\lambda}$ is real or not.

The values of $\chi_{100}$ and $\chi_{160}$ are listed in Table C.9 along with the status "Excess/No excess" or "Dubious" in the last column, derived from these numbers and from a careful inspection of the fields. In addition, PACS $70 \mu \mathrm{m}$ and SPIRE photometry from the literature were included for HIP 61174, HIP 64924, and HIP 88745, and PACS $70 \mu \mathrm{m}$ photometry for HIP 15510. The fluxes and the corresponding references are given at the bottom of Table C.9.

\subsection{Stars with excesses}

After a careful analysis of the corresponding images and surrounding fields, 11 stars of the DUNES_DB sample with $d \leq$ $20 \mathrm{pc}$ show real excesses among those in Table C.9 having $\chi_{100}$ and/or $\chi_{160}>3.0$. They are listed in the upper half of Table 2 .

Eight sources show excess at both 100 and $160 \mu \mathrm{m}$; they are HIP 15510, HIP 16852, HIP 57757, HIP 61174, HIP 64924, HIP 71284, HIP 88745, and HIP 116771.

Two sources, HIP 5862 and HIP 23693, show excess only at $100 \mu \mathrm{m}$ and fluxes at $160 \mu \mathrm{m}$ slightly above the photospheric flux, but not large enough to give $\chi_{160}>3.0$. Consequently, they present a decrease in their SED for $\lambda \geq 100 \mu \mathrm{m}$, similar to those in three stars in DUNES_DU (HIP 103389, HIP 107350, and HIP 114948). An extensive modelling of these three objects was done by Ertel et al. (2012) and their conclusions can be applied to HIP 5862 and HIP 23693.

HIP 113283 shows excess only at $160 \mu \mathrm{m}$; therefore, it is a cold-disc candidate like those discovered and modelled by Eiroa et al. (2011), E13, Krivov et al. (2013), and Marshall et al. (2013).

Among the 11 sources with confirmed excesses, five have been spatially resolved. They are marked with an asterisk in Tables 2 and C.9. Four of them were already known, namely HIP 15510 (Wyatt et al. 2012, Kennedy et al. 2015), HIP 61174 (Matthews et al. 2010; Duchêne et al. 2014), HIP 64924 (Wyatt et al. 2012), and HIP 88745 (Kennedy et al. 2012).

The fifth extended source found in this work is HIP 16852. A two-dimensional Gaussian fit gives full widths at half maximum $(\mathrm{FWHM})$ along the principal axes of $7.7^{\prime \prime} \times 8.5^{\prime \prime}$ at $100 \mu \mathrm{m}$ and $12.2^{\prime \prime} \times 10.5^{\prime \prime}$ at $160 \mu \mathrm{m}$, where those of the PSF along the same directions at each wavelength are $6.9^{\prime \prime} \times 6.8^{\prime \prime}$ and $11.8^{\prime \prime} \times 10.9^{\prime \prime}$, respectively. This implies that the source is marginally resolved at $100 \mu \mathrm{m}$. Thus, HIP 16852 is similar in terms of angular size 
Table 2. Black-body dust temperatures and fractional dust luminosities for the DUNES_DB stars with excesses.

\begin{tabular}{llllll}
\hline \hline HIP & HD & SpT & $\begin{array}{rlll}T_{\mathrm{BB}} & R_{\mathrm{BB}} & L_{\text {dust }} / L_{*} \\
& & (\mathrm{~K}) & (\mathrm{au})\end{array}$ \\
\hline
\end{tabular}

\begin{tabular}{lllccr}
\multicolumn{7}{l}{ Stars with confirmed excesses } \\
\hline 5862 & 7570 & F8 V & 96 & 12 & $2.4 \times 10^{-6}$ \\
$15510^{*}$ & 20794 & G8 V & 32 & 60 & $6.3 \times 10^{-7}$ \\
$16852^{*}$ & 22484 & F9 V & 87 & 18 & $8.4 \times 10^{-6}$ \\
23693 & 33262 & F7 V & 82 & 14 & $2.7 \times 10^{-6}$ \\
57757 & 102870 & F8 V & 38 & 100 & $8.8 \times 10^{-7}$ \\
$61174^{*}$ & 109085 & F2 V & 40 & 107 & $2.2 \times 10^{-5}$ \\
$64924^{*}$ & 115617 & G5 V & 50 & 28 & $2.4 \times 10^{-5}$ \\
71284 & 128167 & F3 V & 111 & 11 & $1.1 \times 10^{-5}$ \\
$88745^{*}$ & 165908 & F7 V & 45 & 52 & $1.1 \times 10^{-5}$ \\
113283 & 216803 & K4 Vp & - & - & $<2.0 \times 10^{-6}$ \\
116771 & 222368 & F7 V & 51 & 54 & $1.1 \times 10^{-6}$
\end{tabular}

Stars with $\chi_{100}$ and/or $\chi_{160}>3.0$ considered dubious excesses

\begin{tabular}{lllccc}
\hline 1599 & 1581 & F9 V & 18 & 264 & $9.6 \times 10^{-7}$ \\
36366 & 58946 & F0 V... & 21 & 405 & $5.7 \times 10^{-7}$ \\
37279 & 61421 & F5 IV-V & 113 & 16 & $1.5 \times 10^{-6}$ \\
44248 & 76943 & F5 V & 27 & 243 & $4.6 \times 10^{-7}$ \\
59199 & 105452 & F0 IV-V & 21 & 358 & $6.9 \times 10^{-7}$ \\
61941 & $110379 J$ & F0 V+... & 30 & 248 & $3.3 \times 10^{-7}$ \\
72659 & 131156 & G8 V+K4 V & $\sim 40$ & 40 & $2.9 \times 10^{-6}$ \\
73695 & 133640 & G2 V+G2 V & 23 & 182 & $1.7 \times 10^{-6}$ \\
77257 & 141004 & G0 V & 33 & 102 & $7.6 \times 10^{-7}$ \\
\hline
\end{tabular}

Notes. ${ }^{(*)}$ denotes that the source is extended. Modified black bodies were fitted for HIP 5862 and HIP 23693 to compute these excesses (see text for details); the $T_{\mathrm{BB}}$ listed in the table correspond to pure black bodies fitted excluding the PACS fluxes at $160 \mu \mathrm{m}$, the corresponding $L_{\text {dust }} / L_{*}$ for those temperatures being $5.22 \times 10^{-6}$ and $4.42 \times 10^{-6}$, respectively. For HIP 61174 two black bodies were fitted to the warm and cold excesses, the warm excess corresponds to a $T_{\mathrm{BB}}=300 \mathrm{~K}$, which implies $L_{\text {dust }} / L_{*}=1.65 \times 10^{-4}$. For HIP $113283-$ a cold disc candidate - there is only one excess flux density at $160 \mu \mathrm{m}$ and a black-body fit is not feasible; the value in italics for the upper limit of $L_{\mathrm{dust}} / L_{*}$ is just indicative and would correspond to a black body of $20 \mathrm{~K}$ normalized to the flux at $160 \mu \mathrm{m}$.

to HIP 15510 , which was also marginally resolved at $100 \mu \mathrm{m}$ by Kennedy et al. $(2015)^{9}$.

Among the excess sources, planets have been detected around HIP 15510 (Pepe et al. 2011), and HIP 64924 (Vogt et al. 2010), see details in Table C.3.

In addition to the excess sources, nine more objects have $\chi_{100}$ and/or $\chi_{160}>3.0$, but are labelled in Table C.9 as "Dubious" because of the complexity of the fields that makes an unambiguous measurement of the fluxes difficult. They are listed in the lower half of Table 2: HIP 1599, HIP 36366, HIP 44248, HIP 37279, HIP 59199, HIP 61941 (all of them Ftype), HIP 72659, HIP 73695 (binaries with a primary of Gtype), and HIP $77257(\mathrm{G})$.

In Table 2, we give the results for $T_{\mathrm{BB}}$, estimated from blackbody fittings, and the fractional luminosity $L_{\text {dust }} / L_{*}$ computed by integrating directly on the one hand the black-body curve

\footnotetext{
9 An inspection of the image at $70 \mu \mathrm{m}$ from the $70 / 160$ waveband combination, which is not used in this work, shows that HIP 16852 is also extended at this wavelength.
}

that best fits the excess, and on the other, the normalized photospheric model. Values are given both for the stars with confirmed and apparent excesses. Typical uncertainties in the black-body temperatures are $\pm 10 \mathrm{~K}$. For all the stars, apart from HIP 61174 , the data involved in the black-body fittings are only the PACS - and SPIRE, if available - flux densities. For HIP 61174, the fit to the warm black body includes all the ancillary photometry between 10 and $30 \mu \mathrm{m}$ (WISE W3 and W4, Akari 18, MIPS 24, IRAS 12 and 25) collected by us and listed in Tables C.6 and C.7.

The values of the fractional luminosity that appear in the table for HIP 5862 and HIP 23693 correspond to modified blackbody fits to all the points showing excess; however, those fits have been used just to integrate the excess fluxes, since the values of $\beta$ - the parameter modifying the pure black body in $\left(\lambda_{0} / \lambda\right)^{\beta}-$ are unreasonably high in both cases, of the order of $\sim 6.0$, making a physical interpretation very difficult; most probably a two-ring disc would be more adequate to model those two cases. The black-body radii computed according to the expression

$R_{\mathrm{BB}}=\left(\frac{278}{T_{\mathrm{BB}}(\mathrm{K})}\right)^{2}\left(\frac{L_{*}}{L_{\odot}}\right)^{1 / 2}$

(see e.g. Backman \& Paresce 1993) are also included in the table.

Figures B.1 and B. 2 show the SEDs of the sources with confirmed and dubious excesses, respectively. In blue we have plotted all the photometry available below $70 \mu \mathrm{m}$, in red the PACS fluxes - and the SPIRE values for some objects, in black the model photosphere normalized, and in green the black-body fits to the excess.

Information for some of these sources has been already reported in other works. Gáspár et al. (2013) compiled a catalogue of Spitzer/MIPS 24 and $70 \mu \mathrm{m}$, and Herschel/PACS $100 \mu \mathrm{m}$ observations that includes the stars analysed in this paper. They confirm excesses for 7 out of the 11 stars listed at the top of Table 2, the exceptions being HIP $57757\left(\chi_{100}=1.01\right)$ and HIP $71284\left(\chi_{100}=3.35\right.$, but no MIPS data available); HIP 113283 is obviously labelled as "no-excess" by these authors because they did not analyse the observation at $160 \mu \mathrm{m}$, the wavelength where we detect an excess. For HIP 116771 they do not provide the PACS $100 \mu \mathrm{m}$ flux and the star is catalogued as a non-excess source according to the MIPS $70 \mu \mathrm{m}\left(\chi_{70}=1.77\right)$.

Chen et al. (2014) analysed and modelled the SEDs of 499 targets that show Spitzer/IRS excesses, including a subset of 420 targets with MIPS $70 \mu \mathrm{m}$ observations. They found that the SEDs for the majority of objects $(\sim 66 \%)$ were better described using a two-temperature model with warm $\left(T_{\text {dust }} \sim 100-500 \mathrm{~K}\right)$ and cold $\left(T_{\text {dust }} \sim 50-150 \mathrm{~K}\right)$ dust populations analogous to zodiacal and Kuiper Belt dust. Four of their excess sources - for which we also find an excess in the far-IR - appear in Chen et al. (2014, VizieR online catalogue J/ApJS/211/25): HIP 5862, HIP 23693, and HIP 64924 are modelled with two-temperature fits, the cold components having $T_{\text {dust }}=94 \pm 8,51 \pm 7$, and $54 \pm 10 \mathrm{~K}$, respectively, whereas HIP 16852 is reproduced by a one-temperature fit with $T_{\text {dust }}=100 \pm 6 \mathrm{~K}$. The single black-body fits for those stars (see Table 2) are 96, 82, 50, and $87 \mathrm{~K}$, respectively, which do not deviate much from the determinations by Chen et al.

Moro-Martín et al. (2015) also reported the detection or nondetection of dust around stars of the DUNES and DEBRIS samples based solely on their measurements of the Herschel/PACS $100 \mu \mathrm{m}$ fluxes. Eight out of the 11 stars we claim as having excess match their positive detections, the exception being 

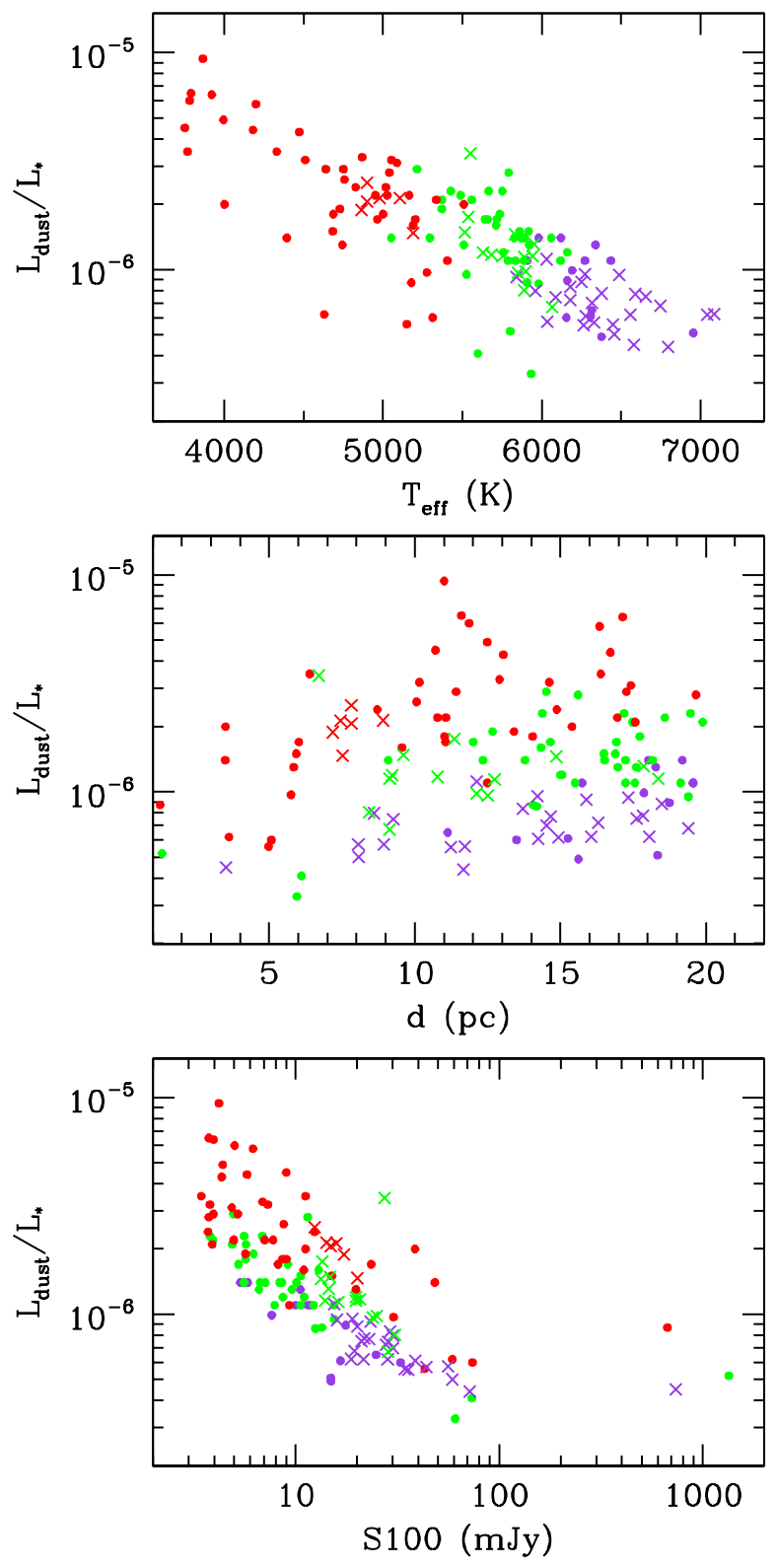

Fig. 4. Upper limits of the fractional luminosity of the dust for the nonexcess sources of the DUNES_DB (crosses) and the DUNES_DU (dots) samples within $20 \mathrm{pc}$, plotted against the effective temperature, distance, and stellar flux at $100 \mu \mathrm{m}$. Violet, green, and red represent F, G, and K stars. See text for details.

HIP 57757, for which they find a $\chi_{100}=2.43$, and HIP 113283 , for which we only detect excess at $160 \mu \mathrm{m}$, as pointed out above; HIP 88745 does not appear in their sample.

Since the observed excess emission of some disc candidates is relatively weak, the possibility of contamination by background galaxies cannot be excluded (E13, Krivov et al. 2013). Sibthorpe et al. (2013) carried out a cosmic variance independent measurement of the extragalactic number counts using PACS $100 \mu \mathrm{m}$ data from the DEBRIS survey. To estimate the probability of galaxy source confusion in the study of debris discs, Table 2 of that paper gives the probabilities of one background source existing within a beam half-width halfmaximum radius (3.4" for PACS $100,5.8^{\prime \prime}$ for PACS 160$)$ of the measured source location for a representative range of excess flux densities.
We have computed the excess flux densities at 100 and $160 \mu \mathrm{m}$ for the 11 discs in Table 2. The smallest values at each wavelength occur for HIP $15510(8.0 \mathrm{mJy})$ and HIP 113283 $(7.8 \mathrm{mJy})$. These values imply the probability of coincidental alignment with a background object of $0.2 \%$ and $1 \%$, respectively. Since the excess flux densities are larger for the remaining targets, the probabilities for them are obviously lower.

In the case of a survey, it is more relevant to ask what the probability is that one or more members of the sample coincide with a galaxy. We have computed the median $1 \sigma$ uncertainties - only considering the rms of the measurement, not the calibration error - of the PACS 100 and $160 \mu \mathrm{m}$ fluxes for the set of 54 stars of the DUNES_DB sample within $20 \mathrm{pc}$, the results being 1.63 and $3.18 \mathrm{mJy}$, respectively. Assuming fluxes for potential background sources of three times those $\sigma$ values at the corresponding wavelengths, we obtain at $100 \mu \mathrm{m}$ a chance of coincidental alignment for a single source of $1.1 \%$. This implies the following binomial probabilities $P(i)$ of having $i$ fakes in the sample of 54 stars: $P(0)=55 \%, P(1)=33 \%, P(2)=10 \%$, $P(3)=2 \%$, and $P(4)=0.3 \%$. For $160 \mu \mathrm{m}$, the chance of coincidental alignment of a single source is $2.9 \%$, which implies $P(0)=21 \%, P(1)=33 \%, P(2)=26 \%, P(3)=13 \%$, and $P(4)=5 \%$. Equation (2) of the paper by Sibthorpe et al. (2013) and the matrices provided in that work were used to compute these estimates.

As we pointed out, 5 of the 11 excess sources found in this work are spatially resolved; therefore, we can consider them to be real debris disc detections. According to the results above, the chances that the remaining six sources were all contaminated by background galaxies are $\sim 0.003 \%$ and $\sim 0.4 \%$ at 100 and $160 \mu \mathrm{m}$, respectively.

\subsection{Stars without excesses}

Figure B.3 shows the SEDs of the DUNES_DB stars within 20 pc without excesses. In Fig. 4 the $3 \sigma$ upper limits of the fractional luminosity of the dust for these sources are plotted as crosses against the effective temperature, distance, and stellar flux at $100 \mu \mathrm{m}$. For the sake of completeness, the non-excess DUNES_DU sources within 20 pc have been also added (dots; see Fig. 8 and Table 12 of E13). Violet, green, and red represent F, G and K stars. The dubious sources are not included. The upper limits have been computed using the PACS $100 \mu \mathrm{m}$ flux densities in expression (4) in Beichman et al. (2006), with a representative $T_{\text {dust }}=50 \mathrm{~K}$.

The plots in Fig. 4 confirm and extend what was already seen in E13. The upper panel shows that the upper limits tend to decrease with $T_{\text {eff }}$, the middle panel shows that for a given distance the hotter the star the lower the upper limit, and the lower panel shows that for a given predicted photospheric flux, which depends both on the spectral type and the distance, the upper limits increase with decreasing effective temperature. The general trend of the $L_{\text {dust }} / L_{*}$ upper limits decreasing with the stellar temperature is expected just from pure black-body scaling considerations and the construction of the DUNES survey. Finer details, however, depend on the particular depth achieved for different spectral types and each individual observation.

The lowest values of the upper limits for the fractional luminosity reached are around $\sim 4.0 \times 10^{-7}$; the median for each spectral type are $7.8 \times 10^{-7}, 1.4 \times 10^{-6}$, and $2.2 \times 10^{-6}$ for $\mathrm{F}, \mathrm{G}$, and $\mathrm{K}$ stars, respectively; the median for the whole sample is $1.4 \times 10^{-6}$.

In Fig. 5 histograms of the significance $\chi_{100}$ for the non-excess (black) and excess (red) sources of the merged 


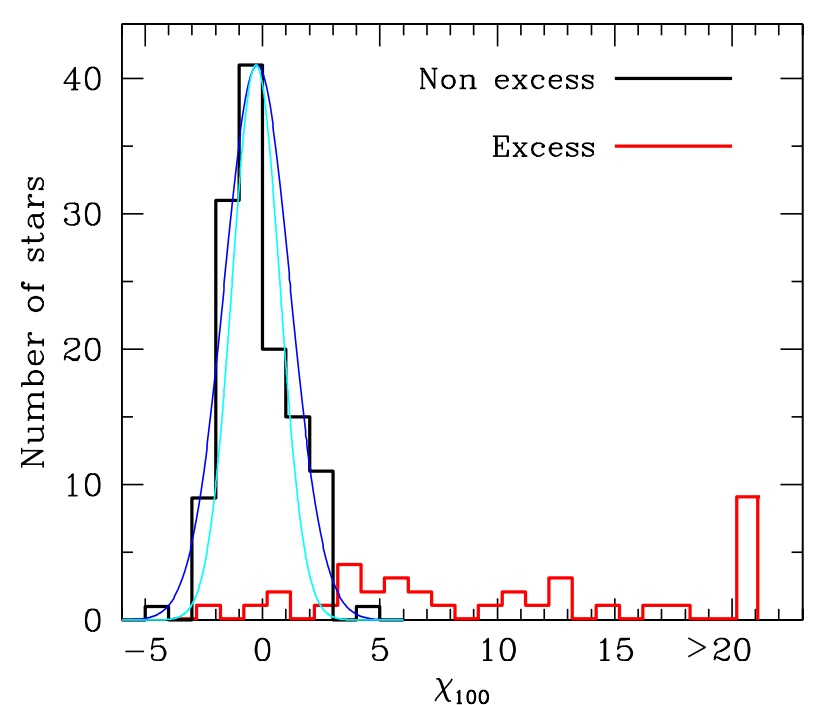

Fig. 5. Plotted in black, the histogram of the $100 \mu \mathrm{m}$ significance $\chi_{100}$ for the non-excess sources of the merged DUNES_DU and DUNES_DB samples with $d \leq 20$ pc (dubious sources of both samples are not included). A Gaussian with $\sigma=1.40$, the standard deviation of the $\chi_{100}$ values for these sources, is plotted in blue. In cyan, we show a Gaussian with $\sigma=1.02$, corresponding to the subsample of non-excess sources with $-2.0<\chi_{100}<2.0$. In red, the distribution of $\chi_{100}$ for the excess sources is also displayed. This figure is an extension of Fig. 6 in E13. See text for details.

DUNES_DU and DUNES_DB samples with $d \leq 20$ pc are plotted. For the non-excess sources, the mean, median, and standard deviation of the $\chi_{100}$-values are $-0.23,-0.33$, and 1.40 , respectively. A Gaussian curve with $\sigma=1.40$ is also plotted. These results are an extension of those already presented in Sect. 7.1 and Fig. 6 of E13, and are quantitatively very similar. The shift to the peak of the distribution to a negative value of $\chi_{100}$ likely reflects the fact that the extrapolation of the model photospheres to the PACS bands using a Rayleigh-Jeans approximation does not take into account the decrease in brightness temperature at $\sim 100 \mu \mathrm{m}$ that occurs in late-type stars, and therefore overestimates the photospheric emission around that wavelength. This was directly observed using DUNES data for $\alpha$ Cen A (Liseau et al. 2013) and $\alpha$ Cen B (Wiegert et al. 2014). We note that the stars in the red histogram with $\chi_{100}<3.0$ are those considered to harbour a cold disc, the excess being present only at $160 \mu \mathrm{m}$.

The fact that the standard deviation of the distribution departs from 1.0 - the expected value assuming a normal distribution - poses a problem already encountered in e.g. E13 and Marshall et al. (2014). One potential origin of this could be the underestimation of the uncertainties either in the PACS $100 \mu \mathrm{m}$ flux, in the photospheric predictions, or both, which would have the effect of increasing the absolute values of $\chi_{100}$ since the term containing the uncertainties is in the denominator of the definition of $\chi_{\lambda}$ (Eq. (1)). The distribution of $\chi_{100}$ would become broader, and $\sigma$ would increase. We have done the experiment of computing the mean, median, and $\sigma$ for the non-excess sources considering only those stars with values of $-2.0<\chi_{100}<2.0$, the goal being to test the central part of the distribution. The results for the three quantities for this subset of stars (108 objects) are -0.28 (mean), -0.34 (median), and $\sigma=1.02$ (Gaussian curve plotted in cyan in Fig. 5). This shows that the problem is more complex than dividing the stars into two bins of "excess" and "non-excess". There must be a spectrum of values with an increasing contribution from dust to the total measurement in the non-excess sample until it hits a threshold and becomes an excess value, which has been set in practice to $\chi_{\lambda}=3.0$. This can have the effect of a departure of the full distribution from normal; instead, narrowing the interval of $\chi_{100}$, ensuring that we deal with a more conservative definition of non-excess sources, seems to have the effect of approaching a normal distribution.

\section{Analysis of the full DUNES sample}

In E13 some conclusions were drawn based solely on the DUNES_DU subsample. In this section we comment on the relevant results that are derived from the analysis of the merged DUNES_DU + DUNES_DB sample, both with $d \leq 15 \mathrm{pc}$ and $d \leq 20$ pc.

\subsection{Excess incidence rates}

Table 3 shows a summary of the excess incident rates in the DUNES_DU, DUNES_DB, and the full sample. In particular, the $d \leq 15$-pc subsample contains $23 \mathrm{~F}, 33 \mathrm{G}$, and $49 \mathrm{~K}$ stars. The incidence rates of excesses are 0.26 (6 objects with excesses out of $23 \mathrm{~F}$ stars), 0.21 (7 out of $33 \mathrm{G}$ stars), and 0.20 (10 out of $49 \mathrm{~K}$ stars), the fraction for the total sample with $d \leq 15 \mathrm{pc}$ being 0.22 ( 23 out of 105 stars). Those percentages do not change significantly if we consider all targets within $d \leq 20 \mathrm{pc}$. We also give the $95 \%$ confidence intervals for a binomial proportion for the corresponding counts according to the prescription by Agresti \& Coull (1998).

As specified in Sect. 4.2, the median of the upper limits of the fractional luminosities per spectral type are $7.8 \times 10^{-7}$, $1.4 \times 10^{-6}$, and $2.2 \times 10^{-6}$ for F, G, and K stars, respectively. As mentioned in E13, these numbers improve the sensitivity reached by Spitzer by one order of magnitude. Our results are to be compared with those found by Trilling et al. (2008, see Table 4 of their paper), where the incidence rates of stars with excess at $70 \mu \mathrm{m}$ as measured by Spitzer/MIPS are $0.18,0.15$, and 0.14 for F, G, and K stars, respectively. The variation of the incidence rates with spectral type is likely directly related to the dependence of the fractional luminosity with $T_{\text {eff }}$ (see Fig. 4).

As we showed in Sect. 2.2, the merged sample with $d \leq$ $15 \mathrm{pc}$ analysed in this paper is complete for F stars and almost complete for $\mathrm{G}$ stars, whereas a number of $\mathrm{K}$ stars have been lost from the parent set of the HIPPARCos catalogue from which the final DUNES sample was drawn, the reason being the observational constraint imposed (background contamination; see Sect. 2.1). In the bottom row of Table 3 we give the expected total number of stars of each spectral type in the HIPPARCos catalogue with $d \leq 15$ pc that would show excess, under the assumption that the individual excess frequencies are those obtained from the full DUNES sample; the numbers that are extrapolations for the $\mathrm{G}$ and $\mathrm{K}$ stars are written in italics. The total incidence rate of excesses for the HIPPARCos subset, namely $0.21_{0.05}^{+0.08}$, does not change with respect to the DUNES result, $0.22_{-0.07}^{+0.09}$, the confidence interval being obviously slightly narrower. Since both $\tau$ Cet and $\epsilon$ Eri are within $15 \mathrm{pc}$ and have debris discs (Lawler et al. 2014; Greaves et al. 2014), if these sources were included in the statistics, the total incidence rate of excesses within $d \leq 15$ pc would be $0.23_{-0.07}^{+0.09}$ ( 25 out of 107 objects).

The results on the incidence rates of excesses from PACS data allows us to push the distribution found from MIPS data, whose minimum detection limit was $L_{\text {dust }} / L_{*} \simeq 4 \times 10^{-6}$ 
Table 3. Summary of stars per spectral type in the DUNES_DU and DUNES_DB samples and frequency of excesses.

\begin{tabular}{|c|c|c|c|c|c|c|c|c|c|c|c|c|}
\hline \multirow[b]{2}{*}{ Sample } & \multicolumn{3}{|r|}{$\mathrm{F}$} & \multicolumn{3}{|r|}{ G } & \multicolumn{3}{|r|}{$\mathrm{K}$} & \multicolumn{3}{|r|}{ FGK } \\
\hline & $\mathrm{T}$ & $\mathrm{E}$ & $\begin{array}{c}\text { Frequency } \\
95 \% \text { interval }\end{array}$ & $\mathrm{T}$ & $\mathrm{E}$ & $\begin{array}{c}\text { Frequency } \\
95 \% \text { interval }\end{array}$ & $\mathrm{T}$ & E & $\begin{array}{c}\text { Frequency } \\
95 \% \text { interval }\end{array}$ & $\mathrm{T}$ & $\mathrm{E}$ & $\begin{array}{c}\text { Frequency } \\
95 \% \text { interval }\end{array}$ \\
\hline$\leq 15$ pc DUNES_DU & 4 & 2 & $\begin{array}{c}0.50 \\
{[0.15-0.85]}\end{array}$ & 19 & 5 & $\begin{array}{c}0.26 \\
{[0.11-0.49]}\end{array}$ & 43 & 9 & $\begin{array}{c}0.21 \\
{[0.11-0.35]}\end{array}$ & 66 & 16 & $\begin{array}{c}0.24 \\
{[0.15-0.36]}\end{array}$ \\
\hline$\leq 20$ pc DUNES_DU & 19 & 4 & $\begin{array}{c}0.21 \\
{[0.08-0.44]}\end{array}$ & 50 & 11 & $\begin{array}{c}0.22 \\
{[0.13-0.35]}\end{array}$ & 54 & 10 & $\begin{array}{c}0.19 \\
{[0.10-0.31]}\end{array}$ & 123 & 25 & $\begin{array}{c}0.20 \\
{[0.14-0.28]}\end{array}$ \\
\hline$\leq 15$ pc DUNES_DB & 19 & 4 & $\begin{array}{c}0.21 \\
{[0.08-0.44]}\end{array}$ & 14 & 2 & $\begin{array}{c}0.14 \\
{[0.03-0.41]}\end{array}$ & 6 & 1 & $\begin{array}{c}0.17 \\
{[0.01-0.58]}\end{array}$ & 39 & 7 & $\begin{array}{c}0.18 \\
{[0.09-0.33]}\end{array}$ \\
\hline$\leq 20$ pc DUNES_DB & 32 & 8 & $\begin{array}{c}0.25 \\
{[0.13-0.42]}\end{array}$ & 16 & 2 & $\begin{array}{c}0.13 \\
{[0.02-0.37]}\end{array}$ & 6 & 1 & $\begin{array}{c}0.17 \\
{[0.01-0.58]}\end{array}$ & 54 & 11 & $\begin{array}{c}0.20 \\
{[0.12-0.33]}\end{array}$ \\
\hline$\leq 15$ pc DUNES_DU+DUNES_DB & 23 & 6 & $\begin{array}{c}0.26 \\
{[0.12-0.47]}\end{array}$ & 33 & 7 & $\begin{array}{c}0.21 \\
{[0.10-0.38]}\end{array}$ & 49 & 10 & $\begin{array}{c}0.20 \\
{[0.11-0.34]}\end{array}$ & 105 & 23 & $\begin{array}{c}0.22 \\
{[0.15-0.31]}\end{array}$ \\
\hline$\leq 20$ pc DUNES_DU+DUNES_DB & 51 & 12 & $\begin{array}{c}0.24 \\
{[0.14-0.37]}\end{array}$ & 66 & 13 & $\begin{array}{c}0.20 \\
{[0.12-0.31]}\end{array}$ & 60 & 11 & $\begin{array}{c}0.18 \\
{[0.10-0.30]}\end{array}$ & 177 & 36 & $\begin{array}{c}0.20 \\
{[0.15-0.27]}\end{array}$ \\
\hline$\leq 15 \mathrm{pc}$ HIPPARcos & 23 & 6 & $\begin{array}{c}0.26 \\
{[0.12-0.47]}\end{array}$ & 42 & 9 & $\begin{array}{c}0.21 \\
{[0.11-0.36]}\end{array}$ & 89 & 18 & $\begin{array}{c}0.20 \\
{[0.13-0.30]}\end{array}$ & 154 & 33 & $\begin{array}{c}0.21 \\
{[0.16-0.29]}\end{array}$ \\
\hline
\end{tabular}

Notes. "T" and "E" mean "Total" and "Excess", respectively.

(Bryden et al. 2006), down to minimum detections around $4 \times$ $10^{-7}$. Figure 6 shows a cumulative distribution of the frequency of excess detections from PACS data as a function of the fractional dust luminosity. The steepness of the distribution decreases at luminosities lower than $\sim 4 \times 10^{-6}$. The extrapolation of a straight line fitted to the bins at the left of that value predicts an incidence of excesses $\sim 0.25$ at $L_{\text {dust }} / L_{*}=10^{-7}$, the current estimate of the Kuiper-belt dust fractional luminosity (Vitense et al. 2012). The cumulative distribution of the frequency of excesses derived from the results shown in Tables 2 and 3 of Bryden et al. (2006, 11 excesses out of 73 stars) is also included in Fig. 6; these were obtained from Spitzer/MIPS observations at $70 \mu \mathrm{m}$, with a detection limit of $L_{\mathrm{dust}} / L_{*} \simeq 4 \times 10^{-6}$. The shape of both distributions is roughly the same in the range $\sim 4 \times 10^{-6}-3 \times 10^{-5}$. A straight line fitted to the bins of our cumulative histogram in that region would predict a larger incidence of excesses $\sim 0.35$, at fractional luminosities of $10^{-7}$. A very similar prediction $(\sim 0.32)$ is obtained from the cumulative histogram by Bryden et al. (2006).

The fact that the Spitzer/MIPS and Herschel/PACS distributions are close to each other in the overlapping range of luminosities is more than a simple consistency check. The similarity might indicate that the dust temperature does not correlate with the dust luminosity in that range. The dust fractional luminosities in Bryden et al. (2006) were based either on a single $70 \mu \mathrm{m}$ measurement or on a combination of $24 \mu \mathrm{m}$ and $70 \mu \mathrm{m}$ fluxes. The dust luminosities in this work were derived from fluxes at longer wavelengths, basically from $70 \mu \mathrm{m}, 100 \mu \mathrm{m}$, and $160 \mu \mathrm{m}$ PACS fluxes. For simplicity, we assume that the Spitzer luminosities were based solely on a $70 \mu \mathrm{m}$ flux and the Herschel values solely on a $100 \mu$ m flux. Under this assumption, the fractional luminosity is directly proportional to the flux at $70 \mu \mathrm{m}$ or $100 \mu \mathrm{m}$ (see Eq. (3) in Bryden et al. 2006). Next, it is obvious that the F70/F100 ratio decreases with decreasing dust temperature. Should the discs with lower fractional luminosities be systematically colder, the F70/F100 flux ratio would decrease with the dust luminosity. This would make the histogram based on F70 flux flatter and conversely, the histogram based on the F100 flux steeper. In the opposite case, i.e. if the discs of low fractional luminosity were systematically warmer, the opposite would be true. In either case, the slopes of the two histograms would differ, but this is not what we see in Fig. 6; instead, they are nearly the same in the $\sim 4 \times 10^{-6}-3 \times 10^{-5}$ luminosity range. Therefore, the dust temperature should be nearly the same for all discs with the dust luminosities in that range.

What implications might this have? The dust temperature is directly related to the disc radius. Assuming that the average stellar luminosity of all stars with discs in that fractional luminosity range is nearly the same, the fact that the dust temperature does not depend on the dust luminosity would automatically mean that the disc radius does not depend on the dust luminosity either. This would imply that it is not the location of the dust-producing planetesimals that primarily determines the disc dustiness. Instead, the disc dustiness must be set by other players in the system, e.g. by the dynamical evolution of presumed planets in the disc cavity.

The bottom line of this analysis is that the flattening of the distribution at low values of $L_{\text {dust }} / L_{*}$ provided by the results of the Herschel observations decreases drastically the predictions of the excess incidence rate at luminosities $\sim 10^{-7}$ compared to those obtained from MIPS results.

\subsection{Debris discs/binarity}

Figure 7 shows the projected binary separation versus disc radius for excess sources in those stars catalogued as binaries in Table C.3 of this work (red symbols) as well as those in E13 (Table 16 of that paper, blue symbols). Squares and diamonds represent confirmed and dubious excess sources, respectively; triangles mark the spectroscopic binary HIP 77257, which has an unknown - but presumably small - separation. Filled and open symbols connected with an arrow represent the black-body disc radius, $R_{\mathrm{BB}}$ computed using Eq. (2) and a more realistic disc radius estimate, $R_{\text {dust }}$, according to Pawellek \& Krivov (2015), respectively, for the same system ${ }^{10}$.

${ }^{10}$ Assuming a composition of astrosilicate (50\%) and ice (50\%), $\Gamma=$ $5.42\left(L_{*} / L_{\odot}\right)^{-0.35}, R_{\mathrm{dust}}=\Gamma R_{\mathrm{BB}}$. 
B. Montesinos et al.: Debris discs around nearby FGK main-sequence stars

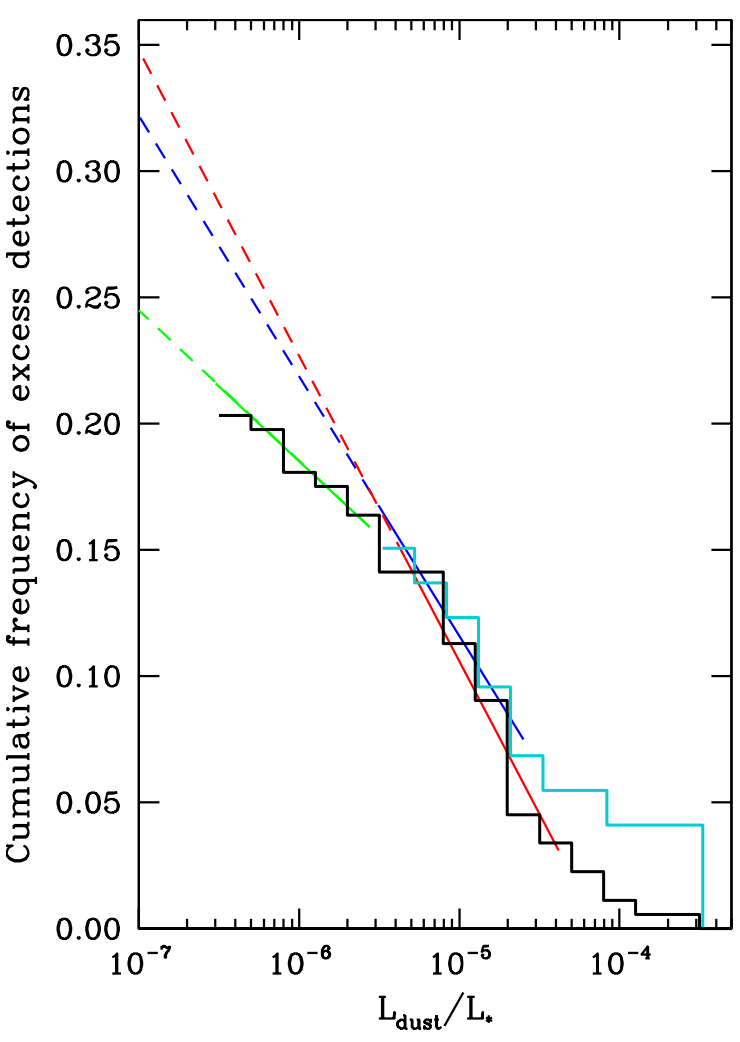

Fig. 6. Cumulative distributions of the frequency of excesses obtained in this work (black) and those obtained from Spitzer/MIPS results Bryden et al. (2006; cyan), both plotted against fractional dust luminosity. The green straight line is a fit to the values of the frequencies at the less steep part of our cumulative distribution. The extrapolation down to $10^{-7}$, the current estimate of the Kuiper-belt fractional luminosity, gives a prediction for the incidence rate of excesses of $\sim 0.25$. The extrapolation from a straight line, plotted in red, fitted to the steeper part of the distribution, that mimics the MIPS results, gives a higher prediction $\sim 0.35$. The prediction from the Bryden et al. (2006) distribution, from the blue straight-line fit, is $\sim 0.32$. See text for details.

The discs above the diagonal straight line are circumprimary, those below the line circumbinary. The grey area roughly marks the discs that are not expected to exist as they would be disrupted by the gravity of the companion (assuming the true distance to be close to the projected one). There is no apparent reason why all the red symbols, which correspond to stars in the DUNES_DB sample, lie in the "circumbinary" region. The ranges of the disc radii in DUNES_DU and DUNES_DB are similar, it is only the distributions of the binary separations that are different (more wide binaries in the DUNES_DU sample); whether a wide or a close binary is found among the excess sources in one of the samples seems to be purely accidental.

\subsection{Debris discs/metallicity}

In Fig. 8, the fractional luminosities $L_{\text {dust }} / L_{*}$ (for the excess sources) and the upper limits (non-excess sources) for the full DUNES sample with $d \leq 20$ pc are plotted against stellar metallicity. It is interesting to note that whereas above the mean metal abundance of the whole sample, $[\mathrm{Fe} / \mathrm{H}]_{\text {mean }}=-0.11$, we find a wide range of values of the fractional luminosity covering more than two orders of magnitude, the discs around stars with $[\mathrm{Fe} / \mathrm{H}]$ below -0.11 seem to show a much narrower interval of $L_{\mathrm{dust}} / L_{*}$, around $\sim 10^{-5}$. Two statistical tests performed on the data, namely the Kolmogorov-Smirnov and the

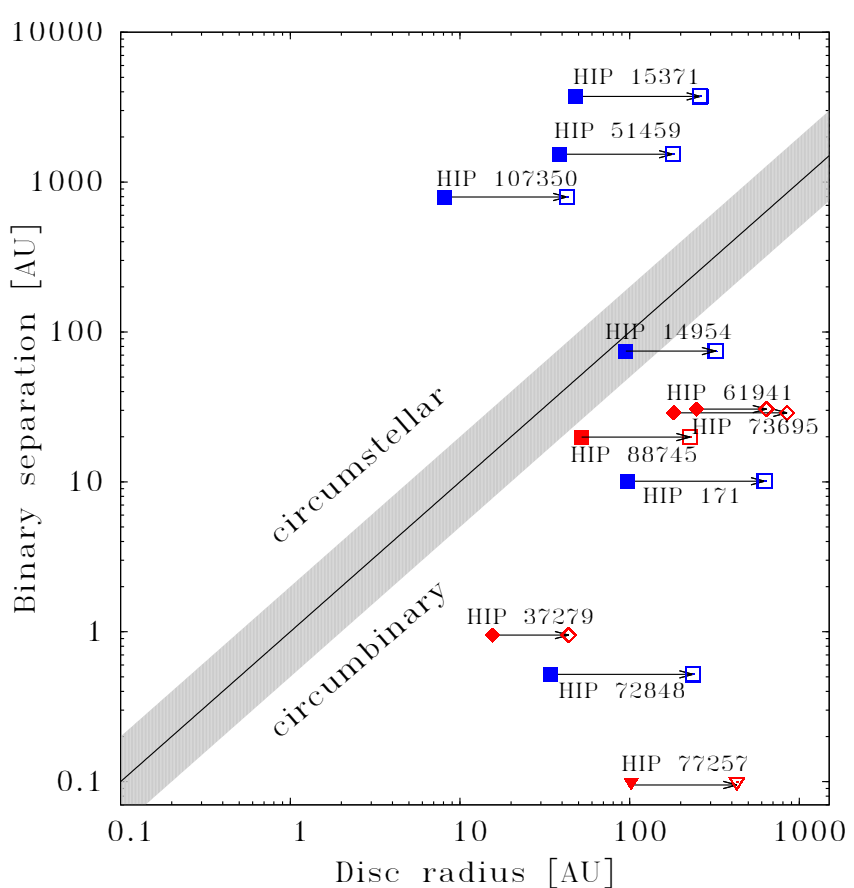

Fig. 7. Projected binary separation plotted against disc radius for excess sources in those stars catalogued as binaries. Red and blue symbols represent objects studied in this work and in E13, respectively. Squares and diamonds are confirmed or dubious excesses. Filled and open symbols represent the value of $R_{\mathrm{BB}}$ and $R_{\text {dust }}$ (see text for details). The inverted triangles at the bottom represent the binary HIP 77257 for which the separation is not known.

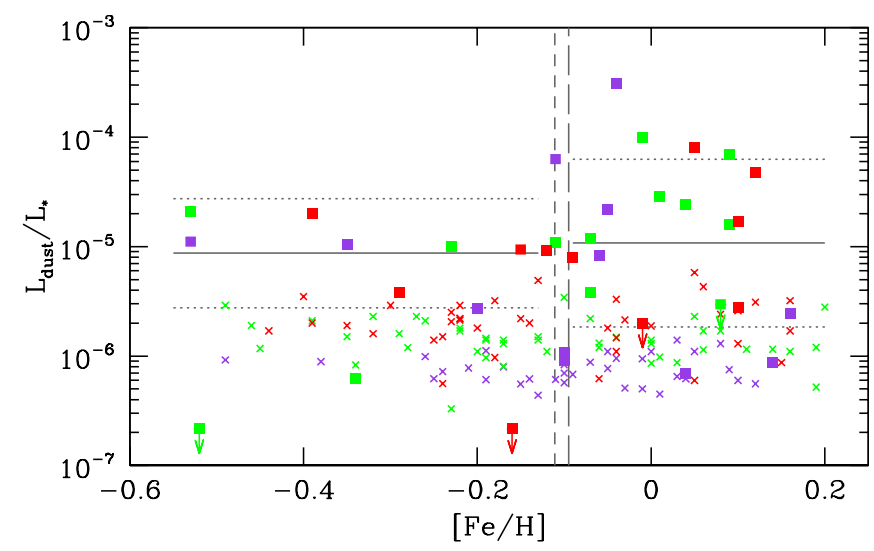

Fig. 8. Fractional dust luminosities $L_{\text {dust }} / L_{*}$ for the DUNES_DU and DUNES_DB stars with excesses (squares) and $d \leq 20 \mathrm{pc}$ (Table 14 in E13 and Table 2 in this work), and upper limits for the non-excess sources (crosses), all of them plotted against metallicity, $[\mathrm{Fe} / \mathrm{H}] . \mathrm{F}, \mathrm{G}$, and $\mathrm{K}$ stars are plotted in violet, green, and red. The short-dashed vertical line marks the mean metal abundance of the whole sample, $[\mathrm{Fe} / \mathrm{H}]=-0.11$, whereas the long-dashed line marks the median, $[\mathrm{Fe} / \mathrm{H}]=-0.095$. The horizontal lines mark the means (solid) of the fractional luminosities for the debris discs of stars showing excess, with $[\mathrm{Fe} / \mathrm{H}] \leq-0.11$ and $>-0.11$, and plus and minus their standard errors (dotted), defined as $\sigma / \sqrt{n}$, where $\sigma$ is the standard deviation; these computations have been done over the values of $\log \left(L_{\mathrm{dust}} / L_{*}\right)$.

Anderson-Darling, state that the result is not statistically significant; therefore, a larger sample is needed to confirm or discard this trend.

Greaves et al. (2006) and Beichman et al. (2006) found that the incidence of debris discs was uncorrelated with metallicity; 
these results have been confirmed by Maldonado et al. (2012, 2015) who find no significant differences in metallicity, individual abundances, or abundance-condensation temperature trends between stars with debris discs and stars with neither debris nor planets. However, Maldonado et al. (2012) have pointed out that there could be a deficit of stars with discs at very low metallicities $(-0.50<[\mathrm{Fe} / \mathrm{H}]<-0.20)$ with respect to stars without detected discs. A recent work by Gáspár et al. (2016), where the correlation between metallicity and debris disc mass is studied, has confirmed the finding by Maldonado et al. (2012) of a deficit of debris-disc-bearing stars over the range $-0.5 \leq[\mathrm{Fe} / \mathrm{H}] \leq$ -0.2 . Out of the full sample analysed in this paper, 166 stars have determinations of their metallicities, all of which are plotted in Fig. 8; the number of stars with excesses at both sides of the median metallicity, $[\mathrm{Fe} / \mathrm{H}]_{\text {median }}=-0.095$, is $16([\mathrm{Fe} / \mathrm{H}]<-0.095)$ and $20([\mathrm{Fe} / \mathrm{H}]>-0.095)$, i.e. a slightly lower proportion on the low-metallicity side. Considering an average uncertainty of \pm 0.05 dex in the metallicities that could move objects from one to the other side of the boundary marked by the median, we can count the number of debris discs around stars with metallicities lower than $[\mathrm{Fe} / \mathrm{H}]_{\text {median }}-0.05$ and higher than $[\mathrm{Fe} / \mathrm{H}]_{\text {median }}+0.05$, the results being $11 / 83$ and $15 / 83$ objects, respectively. The fractions are $0.13_{-0.06}^{+0.09}$ and $0.18_{-0.07}^{+0.10}$, respectively, with the uncertainties corresponding to a $95 \%$ confidence level. Although this result is still not statistically significant, it points in the same direction as the values hinted by Maldonado et al. (2012) and confirmed by Gáspár et al. (2016).

\subsection{Evolutionary considerations}

Figure 9 shows a plot of $\log R_{\mathrm{HK}}^{\prime}$ against $(B-V)$ for the DUNES stars within $20 \mathrm{pc}$, plotted as cyan squares. The stars with excesses are plotted as diamonds with sizes proportional to $\log L_{\text {dust }} / L_{*}$. The regions separating "very inactive", "inactive", "active", and "very active" stars proposed by Gray et al. (2006, see their Fig. 4) are also indicated. This diagram is a qualitative evolutionary picture of FGK main sequence stars in the sense that as the stars evolve, their rotation rates decrease as a consequence of angular momentum loss and therefore their levels of chromospheric activity also decrease (see e.g. Skumanich 1972; Noyes et al. 1984; Rutten 1987).

Out of the 177 stars of the DUNES sample with $d \leq$ $20 \mathrm{pc}, 175$ have $\log R_{\mathrm{HK}}^{\prime}$ data; 119 objects (25 showing excesses, $21.0 \%)$ are in the region of "inactive" or "very inactive" stars $\left(\log R_{\mathrm{HK}}^{\prime}<-4.75\right.$, "old" objects), whereas 56 (11 with excesses, $19.6 \%)$ are in the region of the "active" or "very active" stars $\left(\log R_{\mathrm{HK}}^{\prime}>-4.75\right.$, "young" objects). This means that the incidence of debris discs is similar among inactive and active stars.

The uneven distribution of stars below and above the activity gap is more apparent if we use large samples of field FGK stars to check the relative abundance of active and inactive objects in the solar neighbourhood. Two catalogues have been used to ascertain this point: the Henry et al. (1996) catalogue contains 814 southern stars within $50 \mathrm{pc}$, most of them $\mathrm{G}$ dwarfs. From the Gray et al. (2006) catalogue, which contains stars within $40 \mathrm{pc}$, we have retained those FGK stars with luminosity classes V or V-IV (1270 objects). The stars of both surveys have been plotted as grey crosses in Fig. 9. The bimodal distribution in stellar activity first noted by Vaughan \& Preston (1980) in a sample of northern objects is seen in both catalogues, the percentage of inactive stars below the gap frontier at $\log R_{\mathrm{HK}}^{\prime}=-4.75$ is $73 \%$ (Henry et al. 1996) and 64\% (Gray et al. 2006) for $B-V<0.85$. For the sample in this paper, the percentage of stars below $\log R_{\mathrm{HK}}^{\prime}=-4.75$ is $68 \%$.

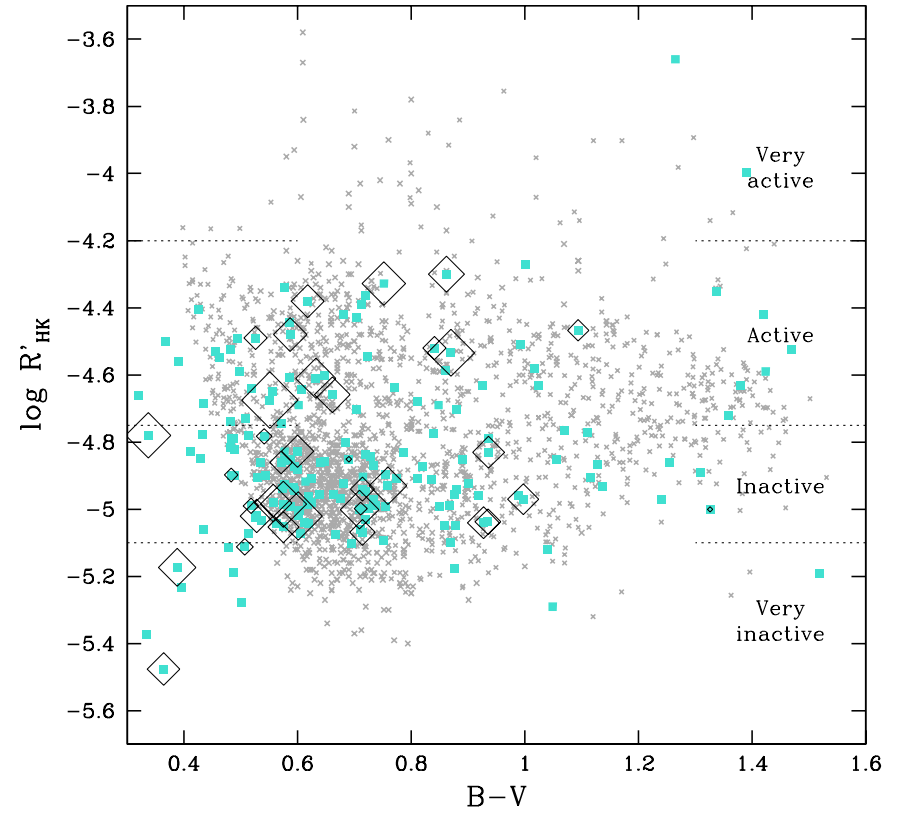

Fig. 9. Diagram of $\log R_{\mathrm{HK}}^{\prime}-(B-V)$ showing the stars of the full DUNES sample with $d \leq 20$ pc (cyan squares). The stars identified as having farIR excess in E13 and in this paper are plotted as diamonds with a size proportional to the value of the fractional dust luminosity. In grey, in the background, the samples of data by Henry et al. (1996) and Gray et al. (2006).

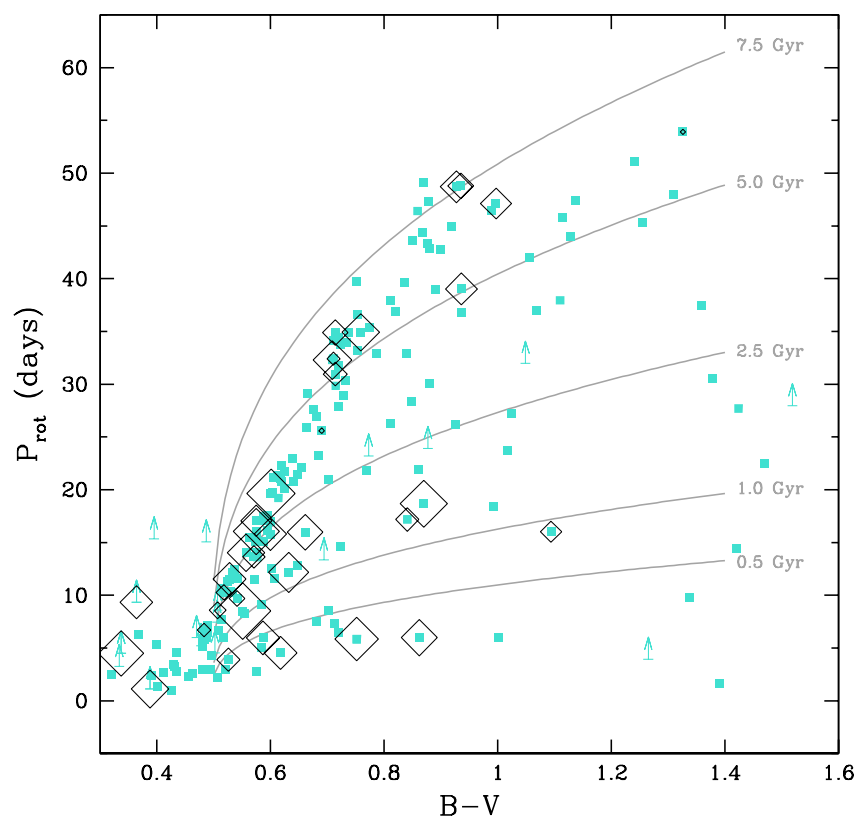

Fig. 10. Diagram of $P_{\text {rot }}-(B-V)$ showing the stars of the full DUNES sample with $d \leq 20 \mathrm{pc}$ (cyan squares). As in Fig. 9 the stars identified as having far-infrared excesses are plotted as diamonds with a size proportional to the value of the fractional dust luminosity. Superimposed are five gyrochrones computed following Mamajek \& Hillenbrand (2008; see Appendix A in this work).

Figure 10 shows the rotation periods (see Table C.2) plotted against the $(B-V)$ colours for the DUNES stars within $20 \mathrm{pc}$. Five gyrochrones corresponding to $0.6,1.0,2.5,5.0$, and $7.5 \mathrm{Gyr}$, computed according to Mamajek \& Hillenbrand (2008) are also included in the graph. The stars with excesses are plotted as black diamonds with sizes proportional to $\log \left(L_{\text {dust }} / L_{*}\right)$. In spite of the problems involved in the estimation of ages, the reliability 
Table 4. Statistics of the dust fractional luminosities.

\begin{tabular}{lcccc}
\hline \hline & \multicolumn{3}{c}{$L_{\text {dust }} / L_{*}$} & \\
\cline { 2 - 4 } & Mean & Median & MAD & $N$ \\
\hline $\log R_{\mathrm{HK}}^{\prime}>-4.75$ (active) & $(5.3 \pm 3.0) \times 10^{-5}$ & $1.7 \times 10^{-5}$ & $1.3 \times 10^{-5}$ & 10 \\
$\log R_{\mathrm{HK}}^{\prime}<-4.75$ (inactive) & $(1.8 \pm 0.6) \times 10^{-5}$ & $9.3 \times 10^{-6}$ & $8.3 \times 10^{-6}$ & 22 \\
$P_{\text {rot }}<10 \mathrm{~d}$ & $(5.0 \pm 3.7) \times 10^{-5}$ & $1.2 \times 10^{-5}$ & $9.7 \times 10^{-6}$ & 8 \\
$10 \mathrm{~d}<P_{\text {rot }}<30 \mathrm{~d}$ & $(2.9 \pm 1.0) \times 10^{-5}$ & $1.4 \times 10^{-5}$ & $1.1 \times 10^{-5}$ & 12 \\
$P_{\text {rot }}>30 \mathrm{~d}$ & $(9.9 \pm 2.9) \times 10^{-6}$ & $8.6 \times 10^{-6}$ & $4.8 \times 10^{-6}$ & 8 \\
\hline
\end{tabular}

Notes. The uncertainties in the means are the standard errors defined as $\sigma / \sqrt{n}$, where $\sigma$ is the standard deviation. The median absolute deviation $(\mathrm{MAD})$ is defined as MAD $=\operatorname{median}[\operatorname{abs}(\mathrm{x}-\operatorname{median}(\mathrm{x}))]$ for a vector $\mathrm{x}$.

of the computation of rotation periods from the chromospheric activity indicator $\log R_{\mathrm{HK}}^{\prime}$ (see Appendix A) makes this diagram a reliable evolutionary scenario of how the sample and the excess sources are distributed.

In Fig. 9 it is apparent that the sizes of the diamonds above the line $\log R_{\mathrm{HK}}^{\prime}=-4.75$ are, on average, larger than those below; the line separates active (younger) from inactive (older) stars. In turn, in Fig. 10 it can be also seen that the average sizes of the symbols decrease as the rotation periods increase, suggesting a decreasing fractional luminosity with increasing stellar age.

To quantify this, we have taken two bins in chromospheric activity, namely stars above and below $\log R_{\mathrm{HK}}^{\prime}=-4.75$, and three bins in rotation period, $P_{\text {rot }}<10 \mathrm{~d}, 10 \mathrm{~d}<P_{\text {rot }}<30 \mathrm{~d}$, and $P_{\text {rot }}>30 \mathrm{~d}$, and have determined means and medians of the dust fractional luminosities of the stars in each bin. The results of this exercise can be seen in Table 4 where the calculations have been carried out for the whole sample $(d \leq 20 \mathrm{pc})$. Stars with upper limits in $L_{\text {dust }} / L_{*}$ (three objects in the DUNES_DU sample and one in DUNES_DB) and with lower limits in $P_{\text {rot }}$ (four objects) have not been included; in the last column $N$ specifies the number of stars used in each case. The effects of decreasing $L_{\text {dust }} / L_{*}$ with decreasing activity and increasing rotation period are apparent. These results show that as the stars get older there seems to be a slow erosion of the mass reservoir and dust content in the disc, with the effect of a decrease in $L_{\text {dust }} / L_{*}$. An extensive and more detailed study on the evolution of debris discs was carried out by Sierchio et al. (2014).

\section{Summary}

The main goal of this paper - and of the DUNES project - is to study the incidence of debris discs around FGK stars in the solar neighbourhood. Data obtained with the ESA Herschel space observatory have been used to characterize the far-IR SED of a sample of objects observed during two Open Time Key Programmes, DUNES and DEBRIS. A sample of 177 stars within 20 pc has been analysed.

Figure 11 shows the fractional dust luminosity, $L_{\text {dust }} / L_{*}$, plotted against the dust temperature, $T_{\text {dust }}$, for the 36 stars for which a far-IR excess has been detected (see Table 14 in E13 and Table 2 in this work). The detection limits for a G5 V star at $20 \mathrm{pc}$, following Bryden et al. (2006), are also included in the graph; the assumed $1 \sigma$ fractional flux accuracies are $20 \%$ for Spitzer/MIPS at $70 \mu \mathrm{m}$ and $10 \%$ for Herschel/PACS at $100 \mu \mathrm{m}$ (i.e. $S / N=10$ ). Fourteen stars are located below the MIPS $70 \mu \mathrm{m}$ curve, showing how Herschel/PACS has pushed the limits

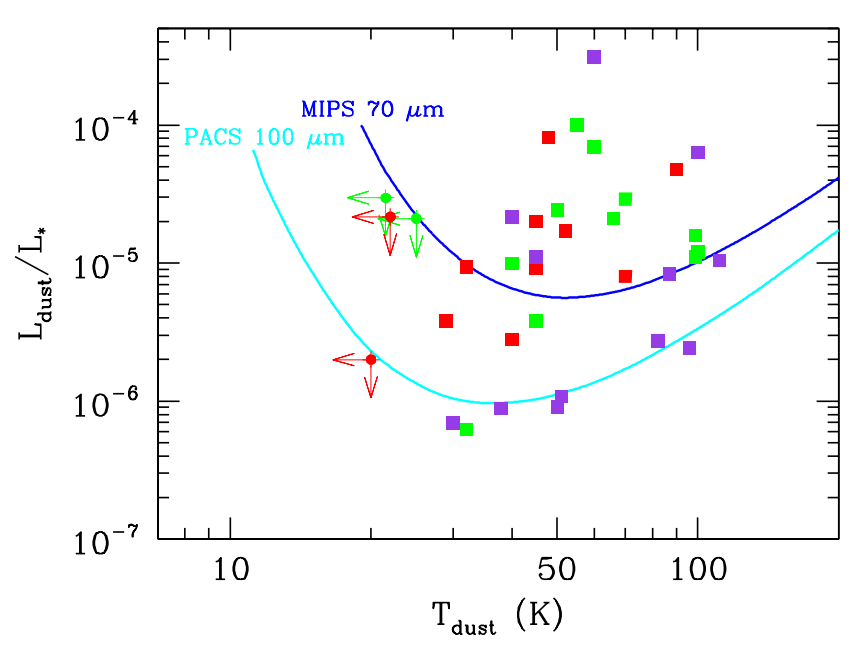

Fig. 11. Diagram of $L_{\text {dust }} / L_{*}-T_{\text {dust }}$ showing the position of the 36 stars, out of the 177 in the DUNES sample within $20 \mathrm{pc}$, for which an excess has been detected at far-IR wavelengths. F, G, and $\mathrm{K}$ stars are plotted in violet, green, and red. The detection limits for a G5 V star at $20 \mathrm{pc}$ for PACS $100 \mu \mathrm{m}$ and MIPS $70 \mu \mathrm{m}$ are also included.

down to fractional luminosities that are a few times that of the Kuiper-belt.

A summary of the main results follows:

1. Herschel/PACS photometry - complemented in some cases with SPIRE data - are provided for the so-called DUNES_DB sample, a set of 54 stars $(32$ F, 16 G, and $6 \mathrm{~K}$ ) observed by DEBRIS for the DUNES consortium. Parameters, ancillary photometry, and details on the multiplicity are also given. The DUNES_DU sample was already analysed in detail in E13 (see Sect. 2, Tables C.1-C.7, and Table C.9).

2. Eleven sources of the DUNES_DB sample show excesses in the PACS 100 and/or $160 \mu \mathrm{m}$ bands (i.e. $\chi_{100}$ and/or $\chi_{160}>$ 3.0). Five of these sources are spatially resolved: four were previously known, whereas HIP 16852 is a new addition to the list of extended sources. This object appears marginally resolved (see Tables 2 and C.9, and Sect. 4.1).

3. The DUNES sample -the merger of DUNES_DU and DUNES_DB- with $d \leq 15$ pc (105 stars) is complete for $F$ stars and almost complete for $G$ stars. The number of $\mathrm{K}$ stars is large enough to provide a solid estimate of the fraction of stars with discs for this spectral type (see Sect. 2.2).

4. The DUNES $d \leq 15$-pc subsample contains $23 \mathrm{~F}, 33 \mathrm{G}$, and $49 \mathrm{~K}$ stars. The incidence rates of debris discs per spectral type are $0.26_{-0.14}^{+0.21}$ ( 6 objects with excesses out of $23 \mathrm{~F}$ stars), $0.21_{-0.11}^{+0.17}$ ( 7 out of $33 \mathrm{G}$ stars) and $0.20_{-0.09}^{+0.14}$ (10 out of 
$49 \mathrm{~K}$ stars), the fraction for all three spectral types together being $0.22_{-0.07}^{+0.08}$ ( 23 out of 105 stars). If $\tau$ Cet and $\epsilon$ Eri, which do not belong to the DUNES sample, were included in the statistics, the total incidence rate of excesses for stars within $d \leq 15$ pc would be $0.23_{-0.07}^{+0.09}$ ( 25 out of 107 objects; see Sect. 5.1 and Table 3).

5. The lowest values reached of the upper limits for the fractional luminosity, $L_{\text {dust }} / L_{*}$, are around $\sim 4.0 \times 10^{-7}$; the median for the whole sample is $1.4 \times 10^{-6}$. These numbers are a gain of one order of magnitude compared with those provided by Spitzer (see Sect. 4.2). Although it may seem obvious, we must emphasize that the excess detection rates reported in this paper are sensitivity limited; therefore, they still represent lower limits of the actual incidence rates (see Sect. 5.1 and the discussion in Fig. 6).

6. There are hints of a different behaviour in the fractional luminosities of the discs at lower and higher metallicities if we split the sample around $[\mathrm{Fe} / \mathrm{H}]_{\text {mean }}=-0.11$ : the former seem to cover a narrower interval of fractional luminosities than the latter. By splitting the sample of stars with determinations of metallicities available (166 objects) around the median $[\mathrm{Fe} / \mathrm{H}]_{\text {median }}=-0.095$, there seems to be a slight deficit of debris discs at lower metallicities (16 discs out of 83 stars) when compared to the number at higher metallicities (20 discs out of 83 stars; see Sect. 5.3, and Fig. 8).

7. Regarding the chromospheric activity, the incidence of debris discs is similar among inactive and active stars. There is a decrease in the average $L_{\text {dust }} / L_{*}$ with decreasing activity/increasing rotation period. Since the stellar activity and the spin-down are proxies of the age, this result suggests that as the stars get older, there seems to be a slow dilution of the dust in the disc with the effect of a decrease in its fractional luminosity (see Sect. 5.4 and Figs. 9 and 10).

Acknowledgements. The authors are grateful to the referee for the careful revision of the original manuscript, and for the comments and suggestions. We also thank Francisco Galindo, Mauro López del Fresno, and Pablo Rivière for their valuable help. B. Montesinos and C. Eiroa are supported by Spanish grant AYA2013-45347-P; they and J.P. Marshall and J. Maldonado were supported by grant AYA2011-26202. A.V. Krivov acknowledges the DFG support under contracts KR 2164/13-1 and KR 2164/15-1. J. P. Marshall is supported by a UNSW Vice-Chancellor's postdoctoral fellowship. R. Liseau thanks the Swedish National Space Board for its continued support. A. Bayo acknowledges financial support from the Proyecto Fondecyt de Iniciación 11140572 and scientific support from the Millenium Science Initiative, Chilean Ministry of Economy, $\mathrm{Nu}$ cleus RC130007. J.-C. Augereau acknowledges support from PNP and CNES. F. Kirchschlager thanks the DFG for finantial support under contract WO 857/151. C. del Burgo has been supported by Mexican CONACyT research grant CB2012-183007.

\section{References}

Agresti, A., \& Coull, B. A. 1998, The American Statistician, 52, 119 Armitage, P. J. 2015, ArXiv e-prints [arXiv: 1509.06382]

Aumann, H. H., \& Probst, R. G. 1991, ApJ, 368, 264

Aumann, H. H., Beichman, C. A., Gillett, F. C., et al. 1984, ApJ, 278, L23

Backman, D. E., \& Paresce, F. 1993, Protostars and Planets III, 1253

Baliunas, S., Sokoloff, D., \& Soon, W. 1996, ApJ, 457, L99

Balog, Z., Müller, T., Nielbock, M., et al. 2014, Exp. Astron., 37, 129

Barnes, S. A. 2007, ApJ, 669, 1167

Beichman, C. A., Bryden, G., Stapelfeldt, K. R., et al. 2006, ApJ, 652, 1674

Bessell, M. S. 1979, PASP, 91, 589

Brott, I., \& Hauschildt, P. H. 2005, in The Three-Dimensional Universe with Gaia, eds. C. Turon, K. S. O’Flaherty, \& M. A. C. Perryman, ESA SP, 576,565

Bryden, G., Beichman, C. A., Trilling, D. E., et al. 2006, ApJ, 636, 1098

Carter, B. S. 1990, MNRAS, 242, 1
Chen, C. H., Mittal, T., Kuchner, M., et al. 2014, ApJS, 211, 25 Cohen, M., Wheaton, W. A., \& Megeath, S. T. 2003, AJ, 126, 1090 Cutri, R. M., et al. 2012, VizieR Online Data Catalog: II/311 Cutri, R. M., et al. 2013, VizieR Online Data Catalog: II/327

Decin, G., Dominik, C., Waters, L. B. F. M., \& Waelkens, C. 2003, ApJ, 598, 636

Duchêne, G., Arriaga, P., Wyatt, M., et al. 2014, ApJ, 784, 148

Duncan, D. K., Vaughan, A. H., Wilson, O. C., et al. 1991, ApJS, 76, 383

Eiroa, C., Marshall, J. P., Mora, A., et al. 2011, A\&A, 536, L4

Eiroa, C., Marshall, J. P., Mora, A., et al. 2013, A\&A, 555, A11

Elias, J. H., Frogel, J. A., Matthews, K., \& Neugebauer, G. 1982, AJ, 87, 1029

Epstein, C. R., \& Pinsonneault, M. H. 2014, ApJ, 780, 159

Ertel, S., Wolf, S., Marshall, J. P., et al. 2012, A\&A, 541, A148

Flower, P. J. 1996, ApJ, 469, 355

Fuhrmann, K. 2008, MNRAS, 384, 173

Gáspár, A., Rieke, G. H., \& Balog, Z. 2013, ApJ, 768, 25

Gáspár, A., Rieke, G. H., \& Ballering, N. 2016, ApJ, submitted http://arxiv.org/abs/1604.07403

Gezari, D. Y., Patricia S. Pitts, P. S., \& Schmitz, M. 2000, Catalog of Infrared Observations, Version 5.1

Glass, I. S. 1975, MNRAS, 171, 19

Gray, R. O. 1998, AJ, 116, 482

Gray, R. O., Corbally, C. J., Garrison, R. F., McFadden, M. T., \& Robinson, P. E. 2003, AJ, 126, 2048

Gray, R. O., Corbally, C. J., Garrison, R. F., et al. 2006, AJ, 132, 161

Greaves, J. S., Fischer, D. A., \& Wyatt, M. C. 2006, MNRAS, 366, 283

Greaves, J. S., Sibthorpe, B., Acke, B., et al. 2014, ApJ, 791, L11

Griffin, M. J., Abergel, A., Abreu, A., et al. 2010, A\&A, 518, L3

Habing, H. J., Dominik, C., Jourdain de Muizon, M., et al. 2001, A\&A, 365, 545

Hall, J. C., Lockwood, G. W., \& Skiff, B. A. 2007, AJ, 133, 862

Hauck, B., \& Mermilliod, M. 1997, VizieR Online Data Catalog: II/215

Hauck, B., \& Mermilliod, M. 1998, A\&AS, 129, 431

Henry, T. J., Soderblom, D. R., Donahue, R. A., \& Baliunas, S. L. 1996, AJ, 111, 439

Holmberg, J., Nordström, B., \& Andersen, J. 2009, A\&A, 501, 941

Kennedy, G. M., Wyatt, M. C., Sibthorpe, B., et al. 2012, MNRAS, 421, 2264

Kennedy, G. M., Matrà, L., Marmier, M., et al. 2015, MNRAS, 449, 3121

Koornneef, J. 1983, A\&AS, 51, 489

Krivov, A. V. 2010, RA\&A, 10, 383

Krivov, A. V., Eiroa, C., Löhne, T., et al. 2013, ApJ, 772, 32

Kroupa, P. 2001, MNRAS, 322, 231

Lawler, S. M., Di Francesco, J., Kennedy, G. M., et al. 2014, MNRAS, 444, 2665

Liseau, R., Montesinos, B., Olofsson, G., et al. 2013, A\&A, 549, L7

Maldonado, J., Eiroa, C., Villaver, E., Montesinos, B., \& Mora, A. 2012, A\&A, 541, A40

Maldonado, J., Eiroa, C., Villaver, E., Montesinos, B., \& Mora, A. 2015, A\&A, 579, A20

Mamajek, E. E., \& Hillenbrand, L. A. 2008, ApJ, 687, 1264

Marshall, J. P., Krivov, A. V., del Burgo, C., et al. 2013, A\&A, 557, A58

Marshall, J. P., Moro-Martín, A., Eiroa, C., et al. 2014, A\&A, 565, A15

Martínez-Arnáiz, R., Maldonado, J., Montes, D., Eiroa, C., \& Montesinos, B. 2010, A\&A, 520, A79

Matthews, B. C., Sibthorpe, B., Kennedy, G., et al. 2010, A\&A, 518, L135

Matthews, B. C., Krivov, A. V., Wyatt, M. C., Bryden, G., \& Eiroa, C. 2014, Protostars and Planets VI, 521

Meibom, S., Barnes, S. A., Platais, I., et al. 2015, Nature, 517, 589

Montesinos, B., Thomas, J. H., Ventura, P., \& Mazzitelli, I. 2001, MNRAS, 326, 877

Moro-Martín, A., Marshall, J. P., Kennedy, G., et al. 2015, ApJ, 801, 143

Mustill, A. J., Veras, D., \& Villaver, E. 2014, MNRAS, 437, 1404

Noyes, R. W., Hartmann, L. W., Baliunas, S. L., Duncan, D. K., \& Vaughan, A. H. 1984, ApJ, 279, 763

Ott, S. 2010, Astronomical Data Analysis Software and Systems XIX, 434, 139

Pawellek, N., \& Krivov, A. V. 2015, MNRAS, 454, 3207

Pecaut, M. J., \& Mamajek, E. E. 2013, ApJS, 208, 9

Pepe, F., Lovis, C., Ségransan, D., et al. 2011, A\&A, 534, A58

Perryman, M. A. C., Lindegren, L., Kovalevsky, J., et al. 1997, A\&A, 323, L49

Phillips, N. M., Greaves, J. S., Dent, W. R. F., et al. 2010, MNRAS, 403, 1089

Pilbratt, G. L., Riedinger, J. R., Passvogel, T., et al. 2010, A\&A, 518, L1

Poglitsch, A., Waelkens, C., Geis, N., et al. 2010, A\&A, 518, L2

Roberge, A., Kamp, I., Montesinos, B., et al. 2013, ApJ, 771, 69

Rutten, R. G. M. 1987, A\&A, 177, 131

Saffe, C., Gómez, M., \& Chavero, C. 2005, A\&A, 443, 609

Salpeter, E. E. 1955, ApJ, 121, 161

Santos, N. C., Israelian, G., \& Mayor, M. 2004, A\&A, 415, 1153

Selby, M. J., Hepburn, I., Blackwell, D. E., et al. 1988, A\&AS, 74, 127 
B. Montesinos et al.: Debris discs around nearby FGK main-sequence stars

Sibthorpe, B., Ivison, R. J., Massey, R. J., et al. 2013, MNRAS, 428, L6

Sierchio, J. M., Rieke, G. H., Su, K. Y. L., \& Gáspár, A. 2014, ApJ, 785, 33

Skiff, B. A. 2009, VizieR Online Data Catalog, 1, 2023

Skumanich, A. 1972, ApJ, 171, 565

Soderblom, D. R. 2010, ARA\&A, 48, 581

Sousa, S. G., Santos, N. C., Mayor, M., et al. 2008, A\&A, 487, 373

Suárez Mascareño, A., Rebolo, R., González Hernández, J. I., \& Esposito, M. 2015, MNRAS, 452, 2745

Takeda, Y., Ohkubo, M., Sato, B., Kambe, E., \& Sadakane, K. 2005, PASJ, 57

Torres, G. 2010, AJ, 140, 1158

Turon, C., Gomez, A., Crifo, F., et al. 1992, A\&A, 258, 74

Trilling, D. E., Bryden, G., Beichman, C. A., et al. 2008, ApJ, 674, 1086
Valenti, J. A., \& Fischer, D. A. 2005, ApJS, 159, 141

Vaughan, A. H., \& Preston, G. W. 1980, PASP, 92, 385

van Leeuwen, F. 2007, A\&A, 474, 653

Vitense, C., Krivov, A. V., Kobayashi, H., \& Löhne, T. 2012, A\&A, 540, A30

Vogt, S. S., Wittenmyer, R. A., Butler, R. P., et al. 2010, ApJ, 708, 1366

Wiegert, J., Liseau, R., Thébault, P., et al. 2014, A\&A, 563, A102

Wittenmyer, R. A., \& Marshall, J. P. 2015, AJ, 149, 86

Wright, C. O., Egan, M. P., Kraemer, K. E., \& Price, S. D. 2003, AJ, 125, 359

Wright, E. L., Eisenhardt, P. R. M., Mainzer, A. K., et al. 2010, AJ, 140, 1868

Wright, J. T., Marcy, G. W., Butler, R. P., \& Vogt, S. S. 2004, ApJS, 152, 261

Wyatt, M. C. 2008, ARA\&A, 46, 339

Wyatt, M. C., Kennedy, G., Sibthorpe, B., et al. 2012, MNRAS, 424, 1206 


\section{Appendix A: Rotation periods and ages}

Noyes et al. (1984) studied the Ca II-Rossby number relation for several sets of mixing-length theory (MLT) models, and found that the tightest correlation between $\log R_{\mathrm{HK}}^{\prime}$ and Ro $=P_{\text {rot }} / \tau_{\mathrm{c}}$ was achieved for the set of models with $\alpha_{\mathrm{MLT}}=1.9$, where $\alpha_{\mathrm{MLT}}$ is the ratio of the mixing length to the pressure scale height in the convection zone. The turnover time, $\tau_{\mathrm{c}}$, is a characteristic time of the convection whose precise definition is

$\tau_{\mathrm{c}}=\frac{H_{\mathrm{p}}(\text { local })}{v_{\text {local }}}$,

where $H_{\mathrm{p}}($ local $)$ is the pressure scale height and $v_{\text {local }}$ is the convective velocity, both quantities computed at a distance of $0.95 H_{\mathrm{p}}$ (base cz) above the base of the outer convective zone, where $H_{\mathrm{p}}($ base $\mathrm{cz})$ is the pressure scale height at the base of the convection zone.

Noyes et al. (1984) derived for that particular value of $\alpha_{\mathrm{MLT}}$ a polynomial fit for the turnover time as a function of $(B-V)$. The fit is given by the expression

$\log \tau_{\mathrm{c}}=\left\{\begin{array}{lr}1.362-0.166 x+0.025 x^{2}-5.323 x^{3}, & x>0 \\ 1.362-0.14 x, & x<0,\end{array}\right.$

where $x=1.0-(B-V)$ and $\tau_{\mathrm{c}}$ is given in days.

Once $\tau_{\mathrm{c}}$ is computed, and provided $\log R_{\mathrm{HK}}^{\prime}$ is known, Noyes et al. (1984) give the expression

$\log$ Ro $=0.324-0.400 y-0.283 y^{2}-1.325 y^{3}$,

which allows a direct estimation of $P_{\text {rot }} ; y$ is defined as $y=5.0+$ $\log R_{\mathrm{HK}}^{\prime}$.

The determination of $P_{\text {rot }}$ from the Rossby number and $\log R_{\mathrm{HK}}^{\prime}$ is valid if $0.4<(B-V)<1.4$ and $-5.1<\log R_{\mathrm{HK}}^{\prime}<$ -4.3 . Similar quantitative relationships can be found in the paper by Mamajek \& Hillenbrand (2008).

In order to test the validity of these relationships when computing $P_{\text {rot }}$ for the stars analysed in this paper, we have taken the sample of 39 FGK stars given by Noyes et al. (1984) and 19 FGK stars by Suárez Mascareño et al. (2015), as well as the Sun, for which the rotation period are known by rotational modulation (first set) and time-series high-resolution spectroscopy (second set). Figure A.1 shows the comparison between the observed rotation periods and the rotation periods estimated following the formalism described above; the Noyes et al. sample is plotted in black and the Suárez-Macareño et al. sample is plotted in light blue. The uncertainties in both $(B-V)$ and $\log R_{\mathrm{HK}}^{\prime}$ have been taken into account when available. Noyes et al. (1984) do not give any uncertainties in the observed periods or in $\log R_{\mathrm{HK}}^{\prime}$, so we have assumed $\sigma\left(\log R_{\mathrm{HK}}^{\prime}\right)=0.05$. It can be seen that the agreement is fairly good, in particular for the Noyes et al. sample. Only two objects, namely HD 25171 and HD 40307, are clear outliers; therefore, we can consider the values of $P_{\text {rot }}$ computed for the DUNES_DB stars following this method, and shown in Table C.2 to be reliable.

Concerning the ages, all the expressions were extracted from the paper by Mamajek \& Hillenbrand (2008). Assuming that $P_{\text {rot }}$ is known, Eqs. (12)-(14) with the revised gyrochronology parameters $a, b, c$, and $n$ in their Table 10 were used to derive the gyrochronology age (in Myr)

$t_{\text {Gyro }}=\left(\frac{P_{\text {rot }}}{a[(B-V)-c]^{b}}\right)^{1 / n}$,

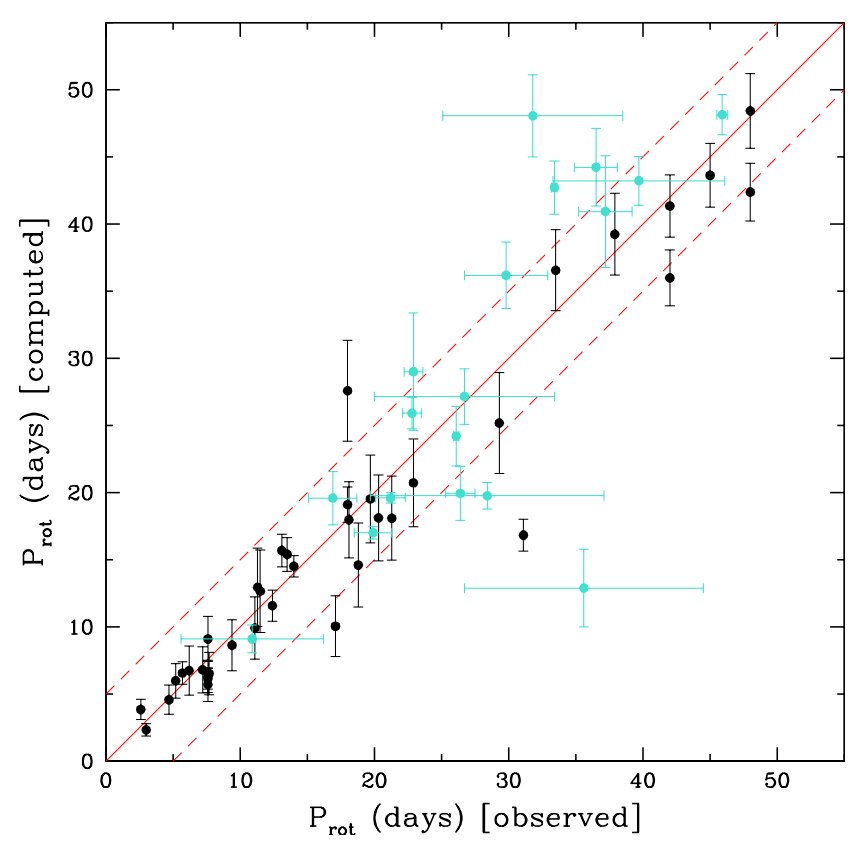

Fig. A.1. Rotation periods estimated according to the formalism described in this Appendix, plotted against the rotation periods for a sample of FGK stars with known values for $P_{\text {rot }}$. Black symbols correspond to stars from Noyes et al. (1984) and cyan symbols to stars from Suárez Mascareño et al. (2015). The dashed lines mark the interval \pm 5 days with respect to the diagonal. See text for details.

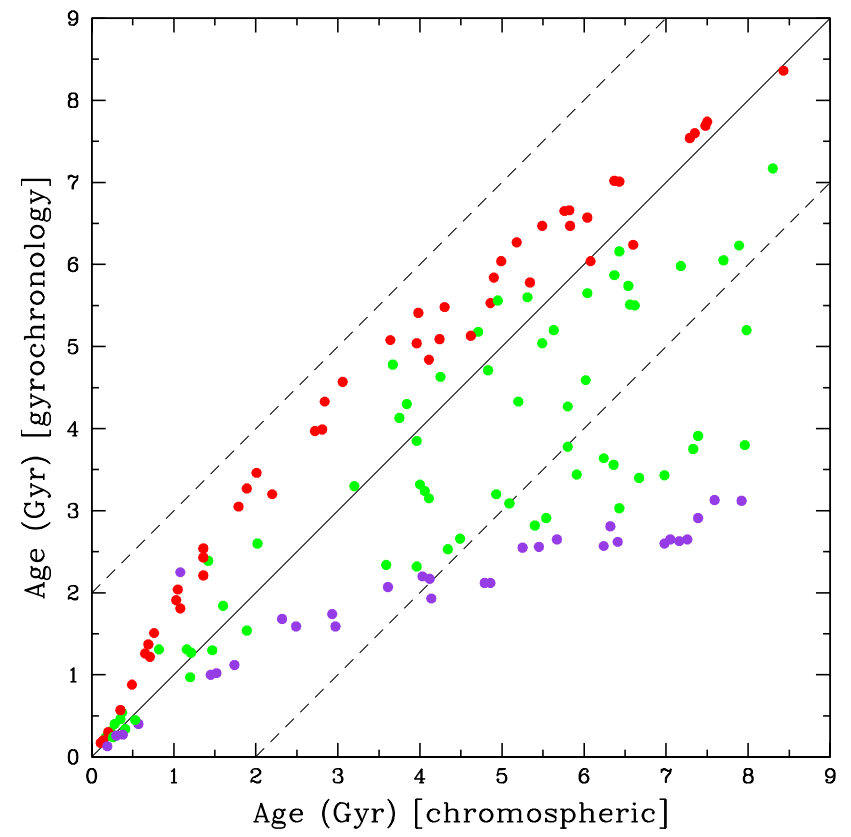

Fig. A.2. Ages for the DUNES_DU and DUNES_DB $(d \leq 20 \mathrm{pc})$ samples estimated by gyrochronology plotted against the chromospheric ages. F, G, and K stars are plotted in violet, green, and red, respectively. The dashed lines mark the interval \pm 2 Gyr with respect to the diagonal.

whereas expression (A.3) was used to estimate the chromospheric age

$\log t_{\mathrm{HK}}=-38.053-17.912 \log R_{\mathrm{HK}}^{\prime}-1.6675\left(\log R_{\mathrm{HK}}^{\prime}\right)^{2}$,

where $t_{\mathrm{HK}}$ is the age in years. The gyrochronology expression is valid for $B-V>0.495$ and the fit to estimate $t_{\mathrm{HK}}$ is only appropriate for $-5.1<\log R_{\mathrm{HK}}^{\prime}<-4.0$. Figure A.2 shows a 
comparison between the ages of the whole DUNES_DU plus DUNES_DB samples estimated using both approaches; obviously, only the stars for which both ages could be estimated are included in the graph. The discrepancies between the ages are clear, the differences being more pronounced for earlier spectral types.

The caveats involving the determination of ages are well known, but its detailed discussion are beyond the scope of this paper (details can be found in e.g. Barnes 2007; Mamajek \& Hillenbrand 2008; Soderblom 2010; Epstein \& Pinsonneault 2014; Meibom et al. 2015 and references therein). The bottom line of this analysis is that the rotation periods establish a reasonable evolutionary proxy for FGK stars, in the sense that the older the star the longer the rotation period, whereas the assignation of an specific age valid in a time span of the order of Gyr has to be taken with caution. 


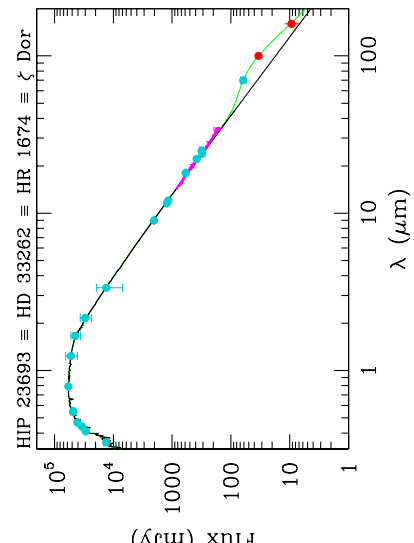

$\left(\kappa_{\Gamma} \mathrm{U}\right) \mathrm{xn}_{\mathrm{H}}$

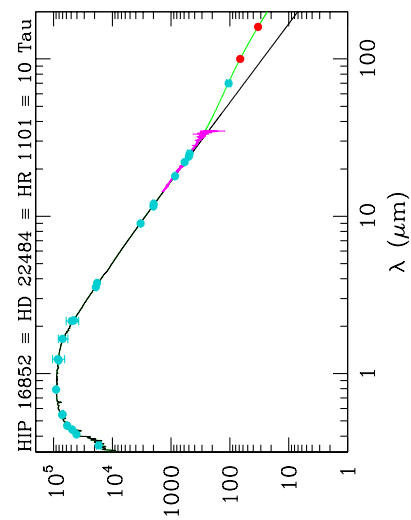

$\left(\kappa_{\Gamma} \mathrm{W}\right) \mathrm{xn}[\mathrm{H}$

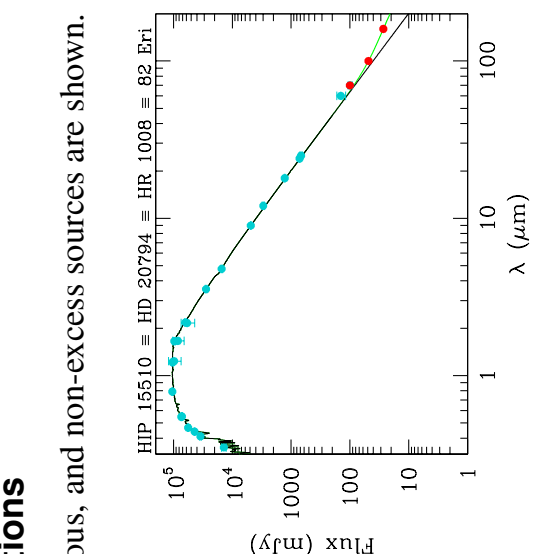

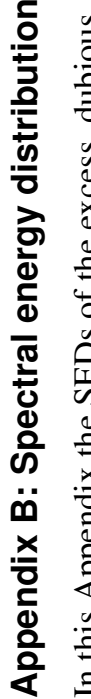
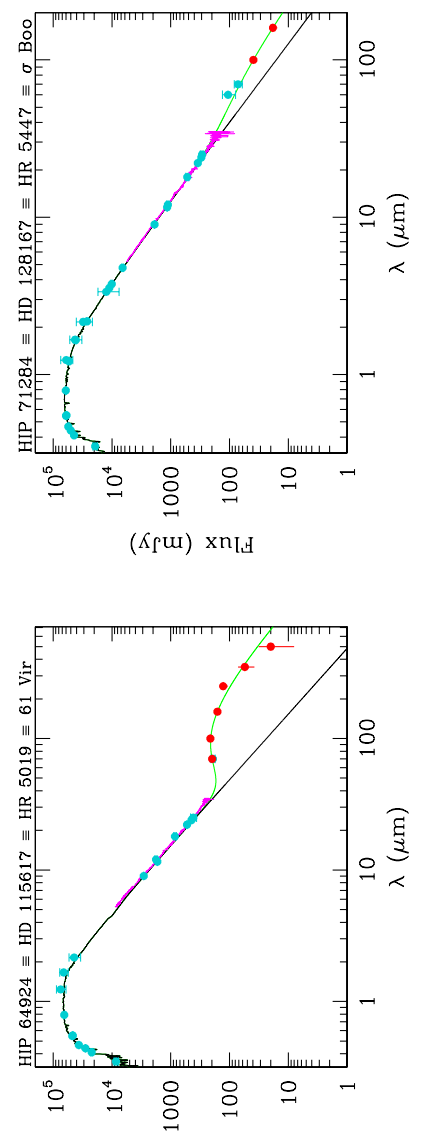

(К К⿴囗十) xnLH

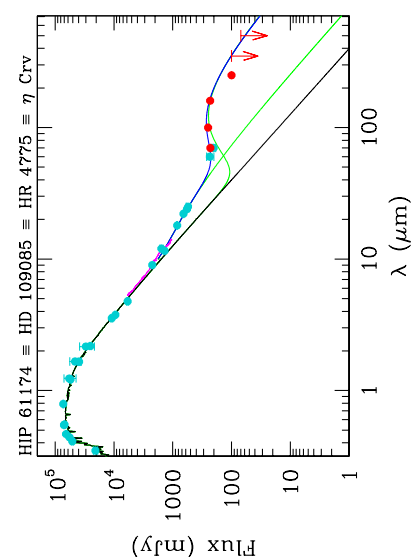

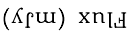

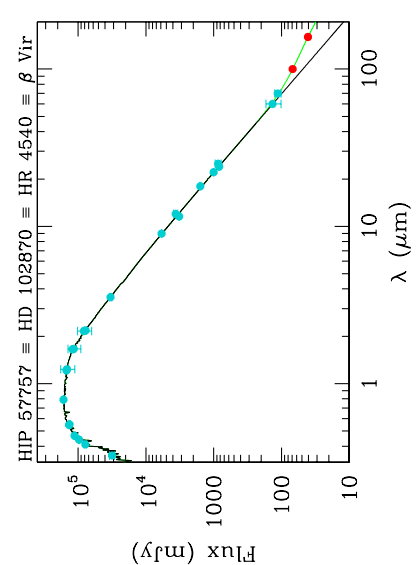

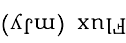
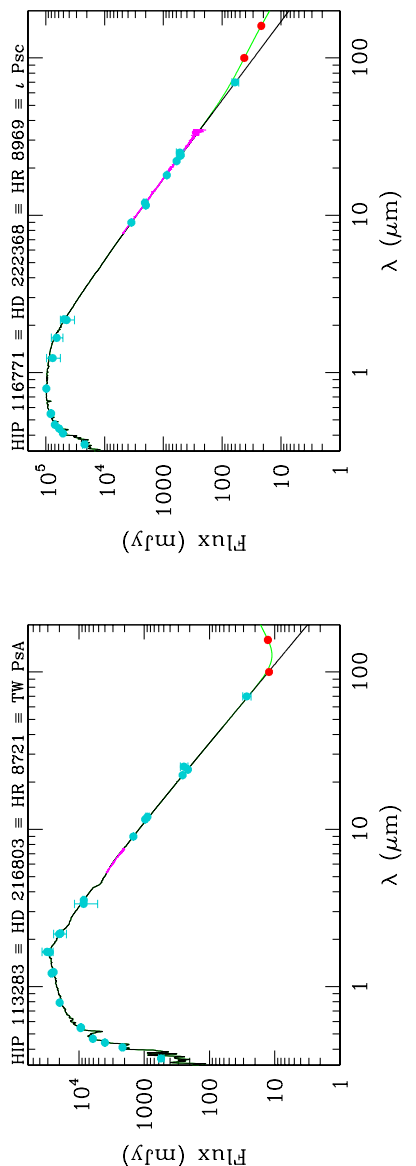

0
0
0
0
0
0
0
0
0
0
0
0
0

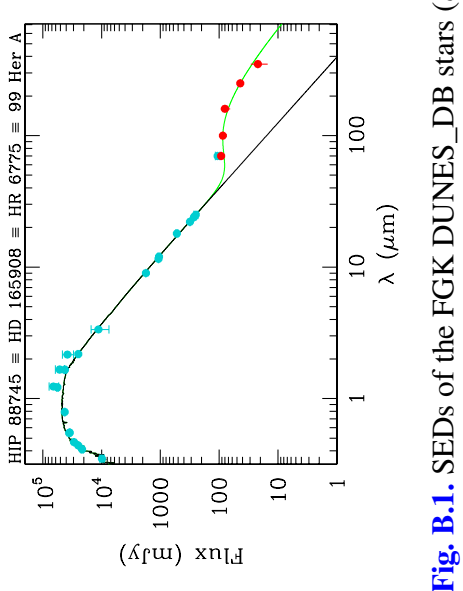


B. Montesinos et al.: Debris discs around nearby FGK main-sequence stars

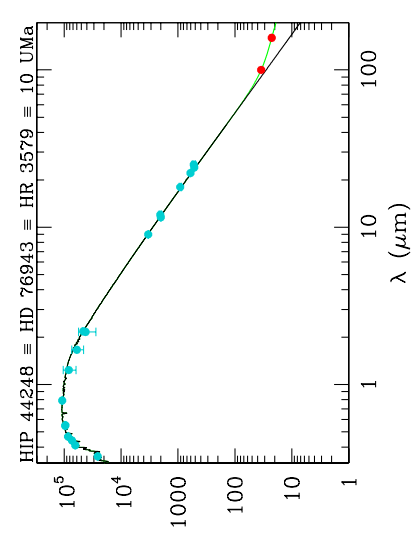

$\left(\kappa_{\text {个 }} \mathrm{U}\right) \mathrm{xn}$

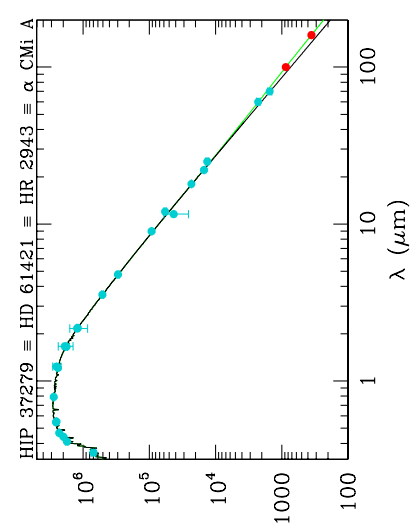

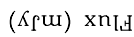

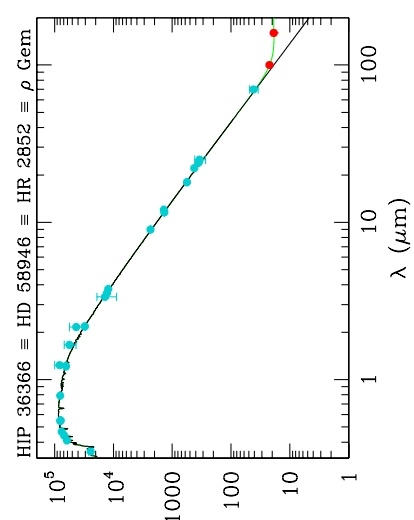

$\left(\Lambda_{\Gamma} \mathrm{U}\right) \mathrm{xn}$ LU

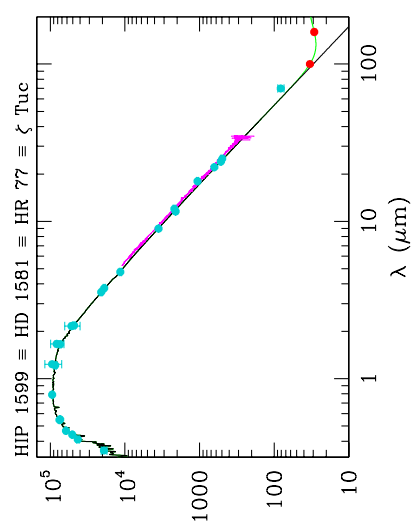

(К 个
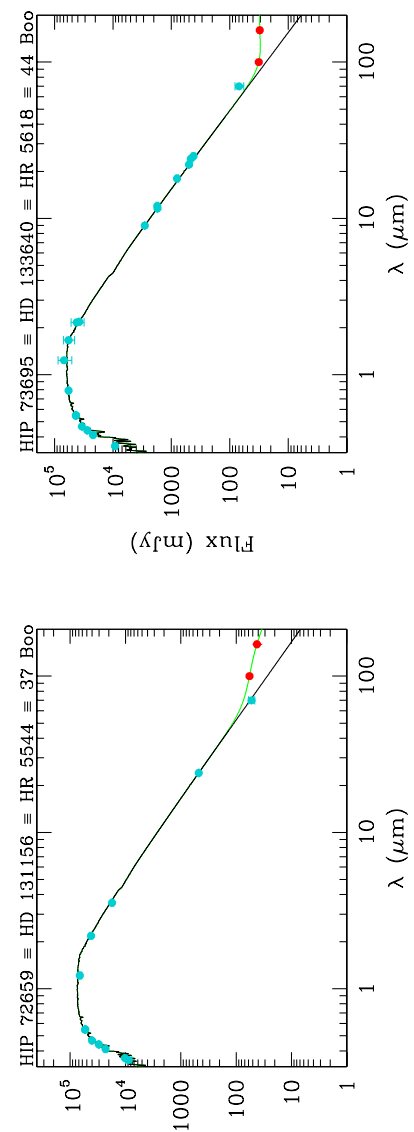

$\left(\kappa_{\Gamma} \mathrm{W}\right) \mathrm{xn}_{\mathrm{IH}}$

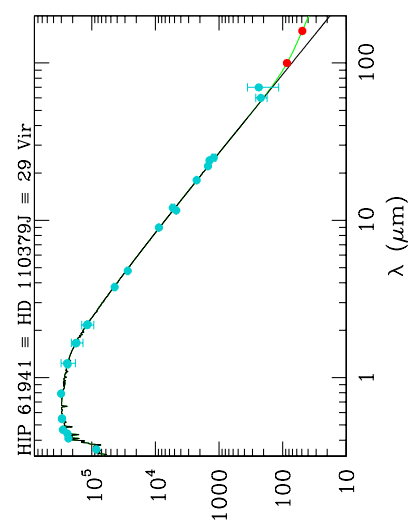

$\left(\Lambda_{\Gamma} \mathrm{W}\right) \mathrm{xn}_{\mathrm{LH}}$

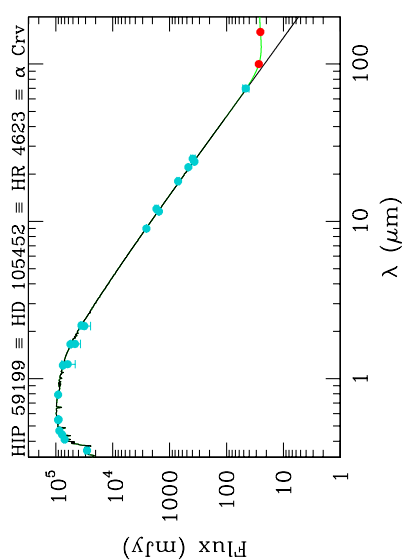

$\left(\kappa_{\Gamma} \mathrm{UU}\right) \mathrm{xn}_{[\mathrm{H}}$ 


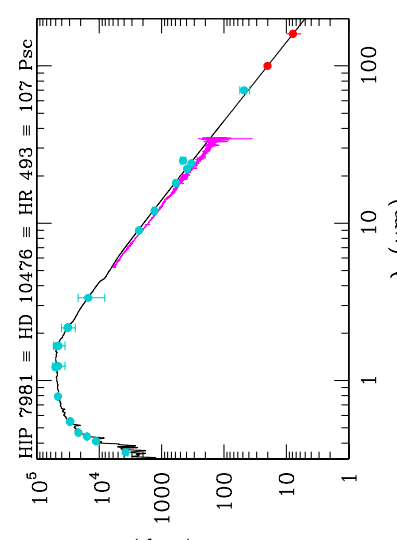

$\left(\kappa_{\Gamma} U\right) \times n_{L H}$

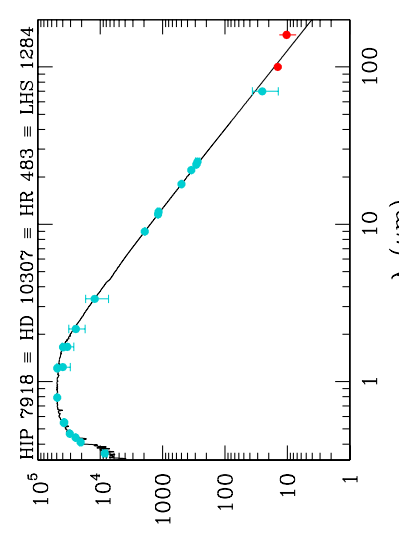

$\left(\kappa_{\Gamma} \mathrm{W}\right) \times n_{\text {IH }}$

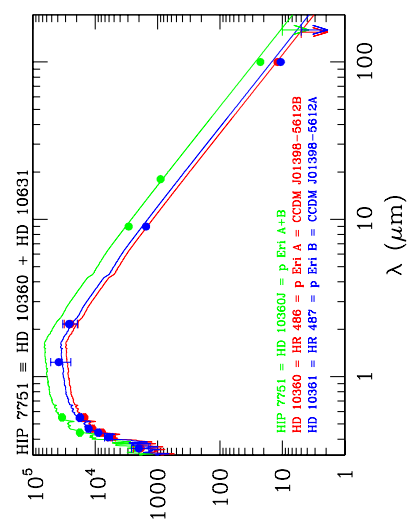

$\left(\kappa_{\uparrow} w\right) \times n_{L H}$

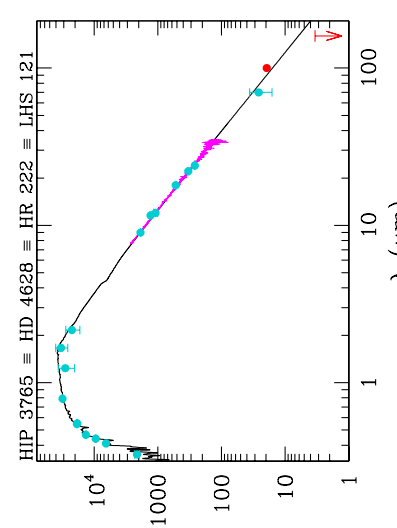

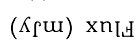

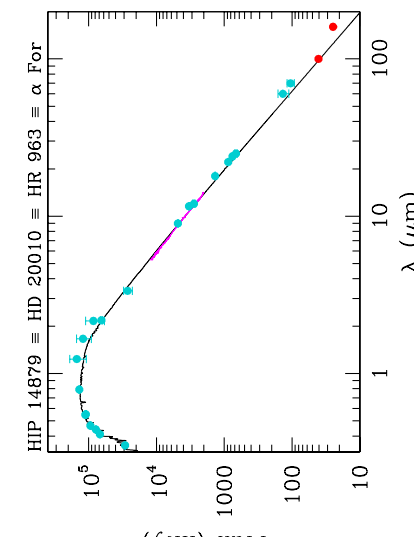

$\left(\kappa_{\Gamma} \mathrm{w}\right) \mathrm{xn}_{\mathrm{LH}}$

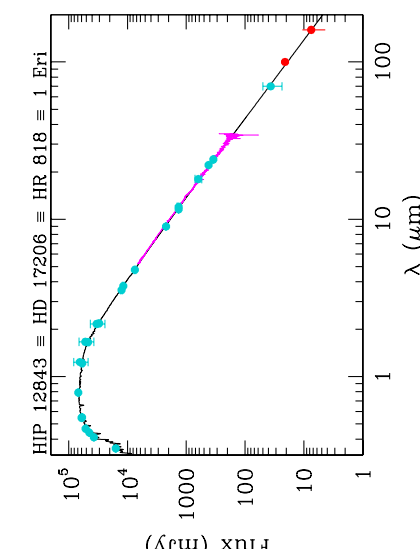

$\left(\kappa_{\Gamma} \mathrm{U}\right) \mathrm{xn}_{\mathrm{LH}}$

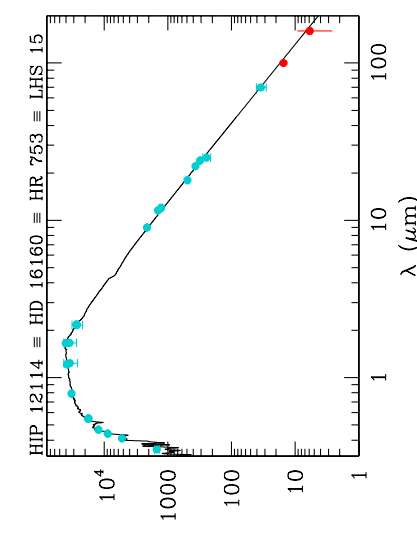

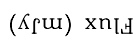

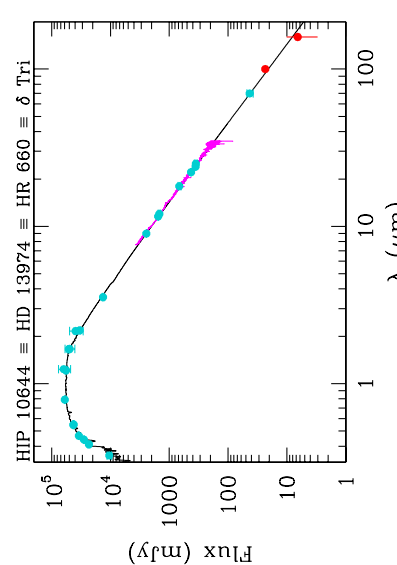

$\left(\kappa_{\Gamma} w\right) \times n_{L, 4}$

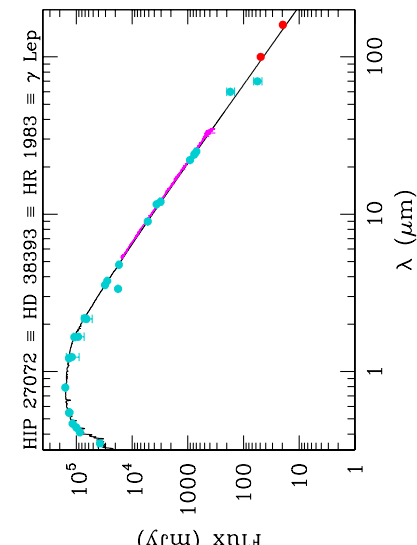

(К К

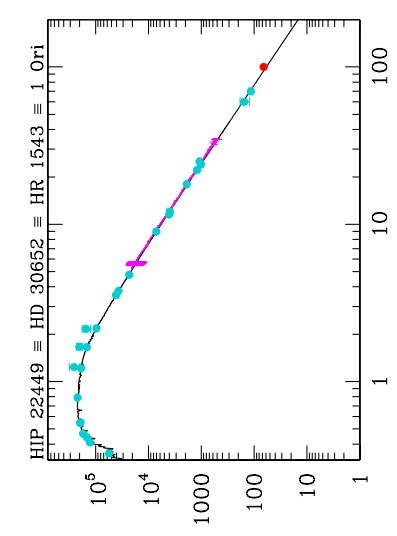

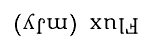

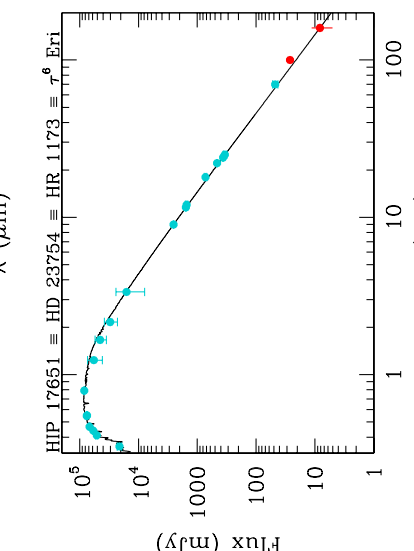

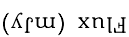
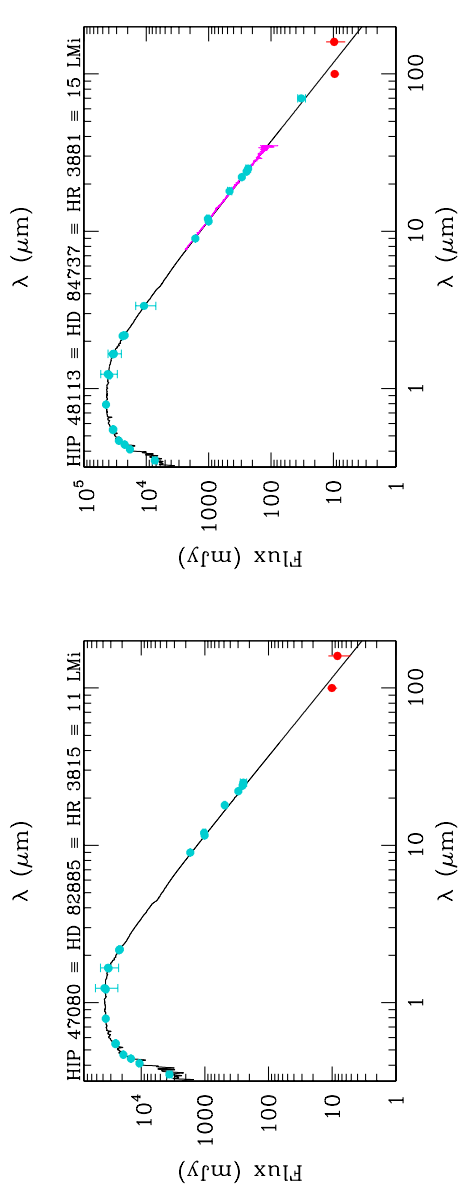

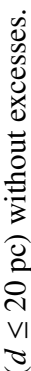
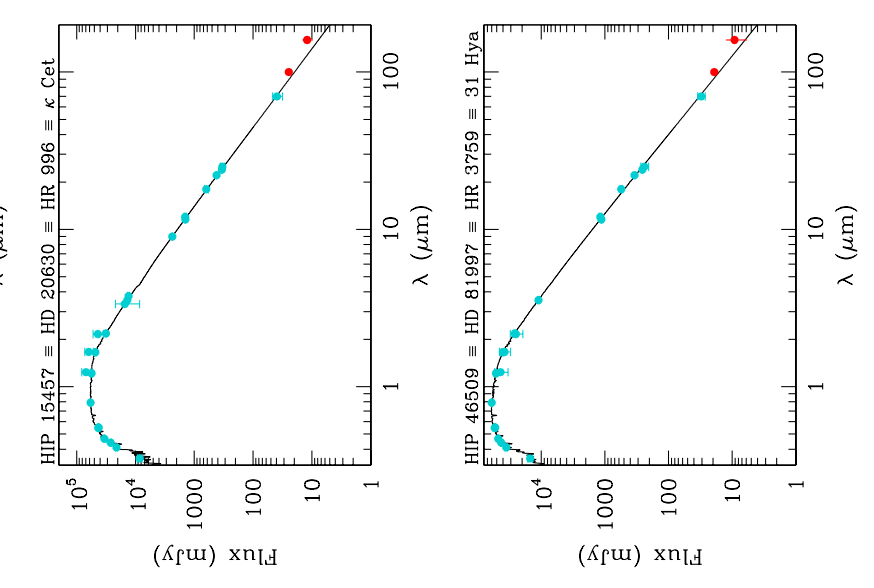

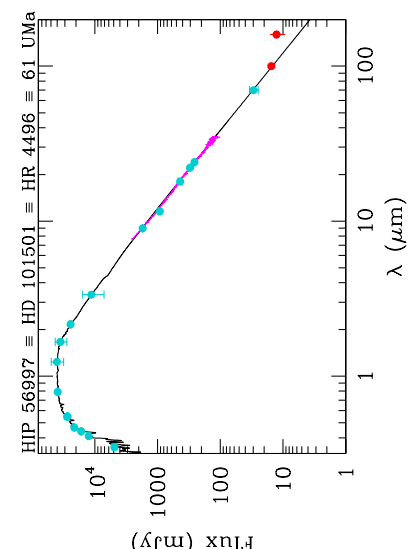

(К К⿴囗) xnIH 
B. Montesinos et al.: Debris discs around nearby FGK main-sequence stars

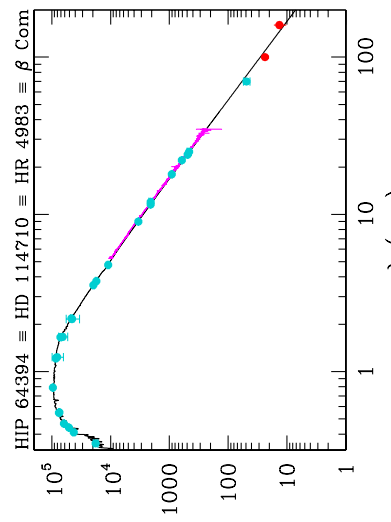

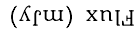

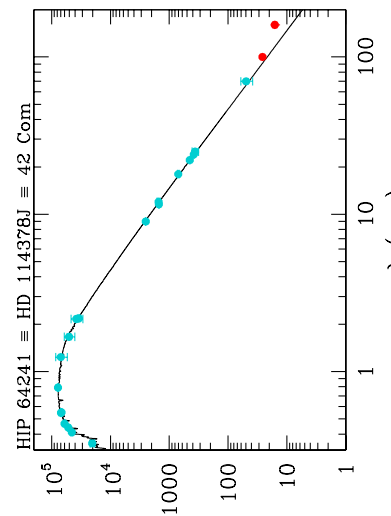

$\left(\kappa_{\Gamma} \mathrm{W}\right) \times n_{[H}$

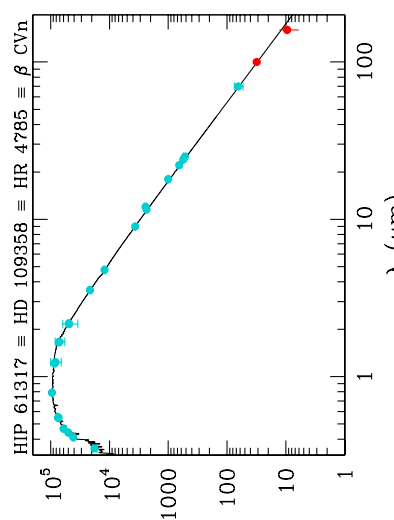

$\left(\kappa_{\uparrow} \mathrm{w}\right) \times n_{L H}$

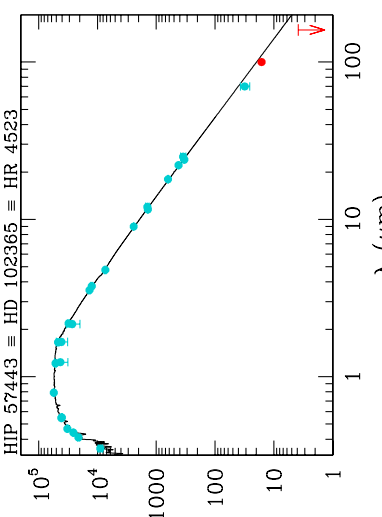

(К К

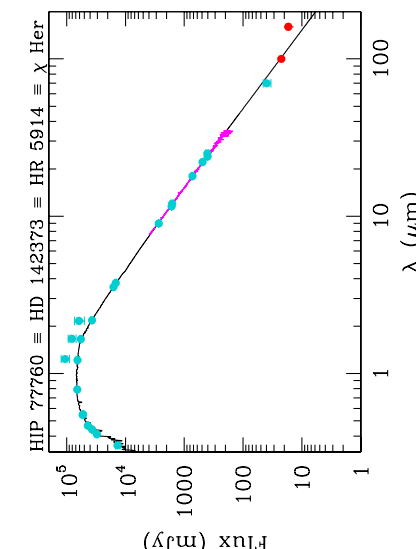

$\left(\kappa_{\text {PU) }}\right) \mathrm{xn}_{\mathrm{LH}}$

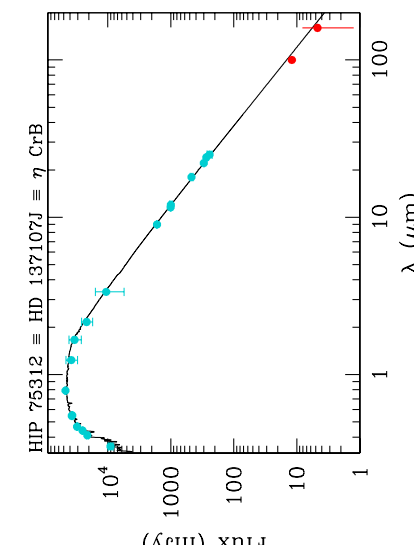

$\left(\kappa_{\Gamma} \mathrm{W}\right) \times n_{L H}$

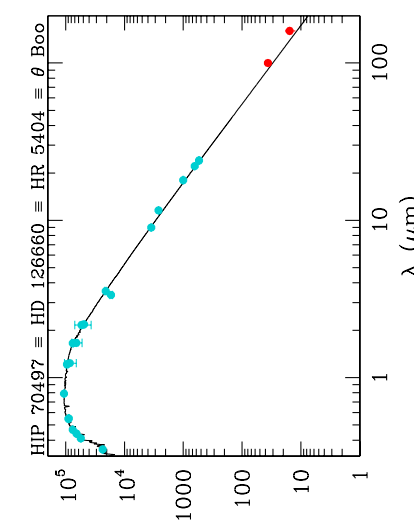

$\left(\kappa_{\uparrow} w\right) \times n_{L H}$

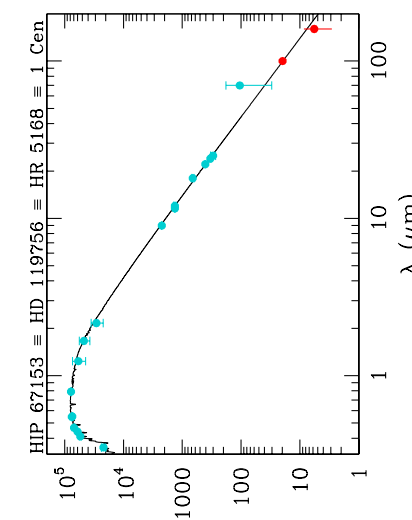

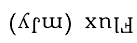

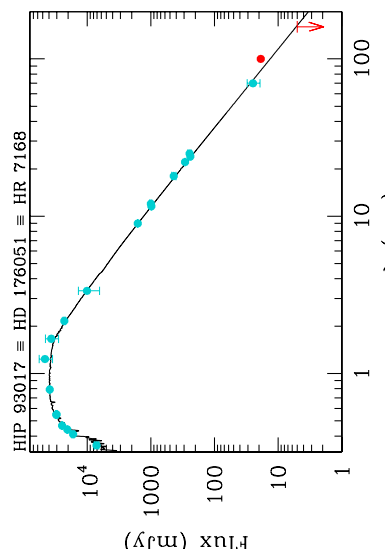

$\left(\kappa_{\Gamma} \mathrm{U}\right) \mathrm{xn} \mathrm{n}_{\mathrm{H}}$

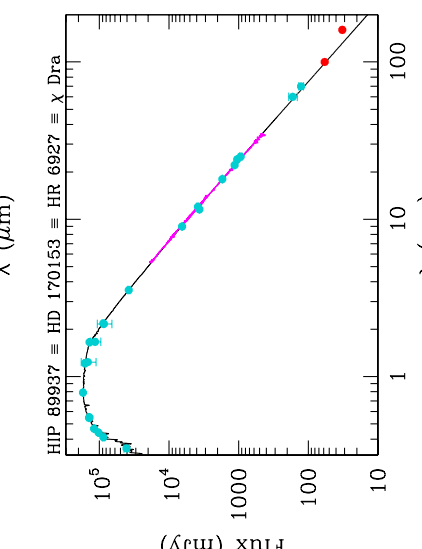

$\left(\kappa_{\Gamma} \mathrm{W}\right) \times n_{L H}$

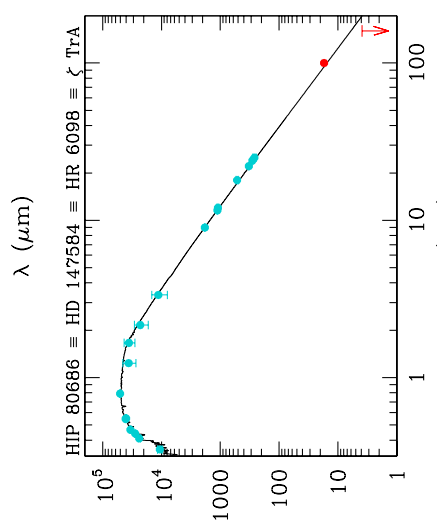

$\left(\kappa_{\Gamma} \mathrm{w}\right) \mathrm{xn}_{\mathrm{H}}$
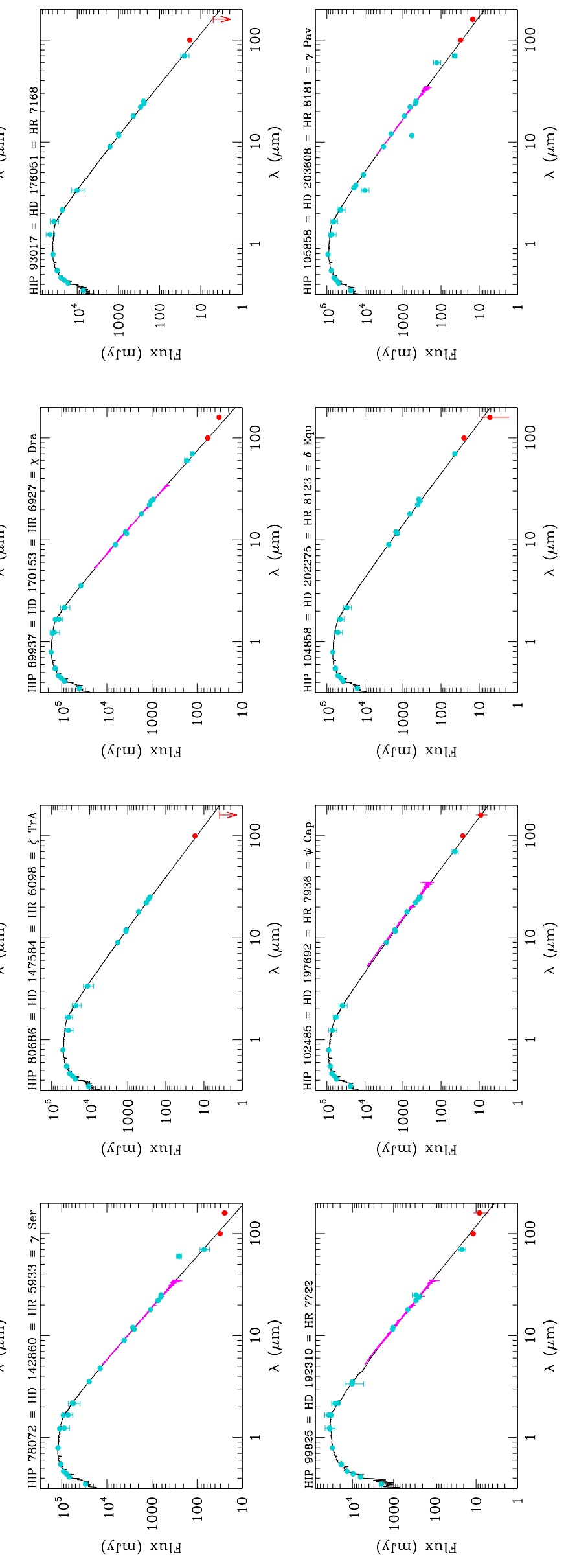

فํ. 
A\&A 593, A51 (2016)
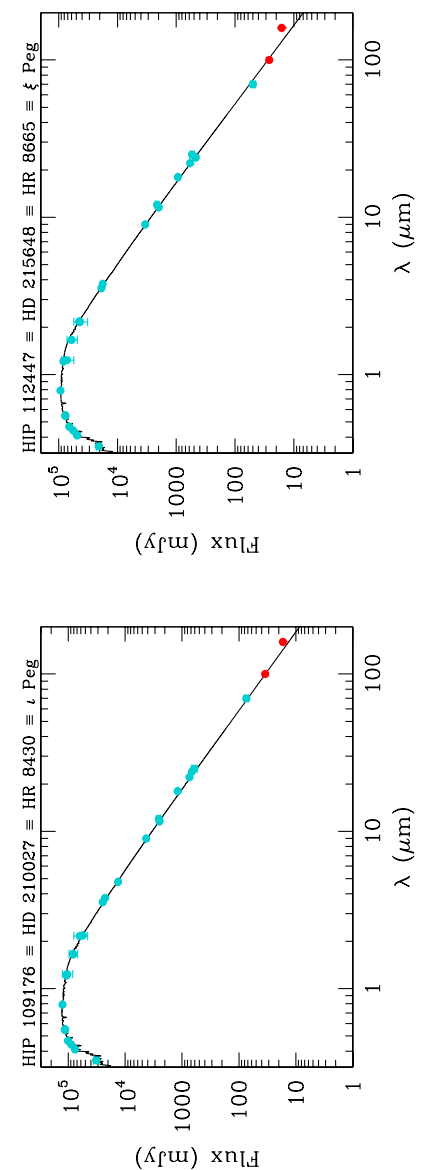

فํ.

A51, page 22 of 31 
B. Montesinos et al.: Debris discs around nearby FGK main-sequence stars

\section{Appendix C: Additional material}

Table C.1. Subsamples of DUNES_DB stars with $d \leq 15 \mathrm{pc}$ and $15 \mathrm{pc}<d \leq 20$ pc.

\begin{tabular}{|c|c|c|c|c|c|c|c|c|}
\hline HIP & HD & Other ID & SpT (HIPPARCOS) & SpT range & \multicolumn{2}{|c|}{ ICRS (2000) } & $\pi($ mas $)$ & $d(\mathrm{pc})$ \\
\hline \multicolumn{9}{|c|}{ FGK stars $(d \leq 15 \mathrm{pc})$} \\
\hline 1599 & 1581 & $\zeta$ Tuc & F9V & F9.5V, F9V-G0V & 002004.260 & -645229.25 & $116.46 \pm 0.16$ & $8.59 \pm 0.01$ \\
\hline 3765 & 4628 & LHS 121 & $\mathrm{~K} 2 \mathrm{~V}$ & $\mathrm{~K} 2.5 \mathrm{~V}, \mathrm{~K} 2 \mathrm{~V}$ & 004822.977 & +051650.21 & $134.14 \pm 0.51$ & $7.45 \pm 0.03$ \\
\hline 7751 & $10360 \mathrm{~J}$ & p Eri & $\mathrm{K} 0 \mathrm{~V}$ & $\mathrm{~K} 0 \mathrm{~V}, \mathrm{~K} 1-\mathrm{K} 3 \mathrm{~V}$ & 013947.540 & -561147.10 & $127.84 \pm 2.19$ & $7.82 \pm 0.13$ \\
\hline 7918 & 10307 & HR 483 & $\mathrm{G} 2 \mathrm{~V}$ & $\mathrm{G} 1.5 \mathrm{~V}, \mathrm{G} 1 \mathrm{~V}$ & 014147.143 & +423648.12 & $78.50 \pm 0.54$ & $12.74 \pm 0.09$ \\
\hline 7981 & 10476 & 107 Psc & $\mathrm{K} 1 \mathrm{~V}$ & $\mathrm{~K} 1 \mathrm{~V}, \mathrm{~K} 0 \mathrm{~V}-\mathrm{K} 1 \mathrm{~V}$ & 014229.762 & +201606.60 & $132.76 \pm 0.50$ & $7.53 \pm 0.03$ \\
\hline 10644 & 13974 & $\delta$ Tri & G0V & G0.5V, F9V-G0V & 021703.230 & +341327.23 & $92.73 \pm 0.39$ & $10.78 \pm 0.05$ \\
\hline 12114 & 16160 & LHS 15 & $\mathrm{~K} 3 \mathrm{~V}$ & $\mathrm{~K} 3 \mathrm{~V}$ & 023604.895 & +065312.75 & $139.27 \pm 0.45$ & $7.18 \pm 0.02$ \\
\hline 12843 & 17206 & 1 Eri & F5/F6V & F6V, F5V-F8V & 024506.187 & -183421.21 & $70.32 \pm 1.83$ & $14.22 \pm 0.37$ \\
\hline 14879 & 20010 & $\alpha$ For & F8V & F6V, F6V-F8V & 031204.527 & -285915.43 & $70.24 \pm 0.45$ & $14.24 \pm 0.09$ \\
\hline 15457 & 20630 & $\kappa$ Cet & G5Vvar & G5Vv, G5V & 031921.696 & +032212.72 & $109.41 \pm 0.27$ & $9.14 \pm 0.02$ \\
\hline 15510 & 20794 & e Eri & G8V & G8V & 031955.651 & -430411.22 & $165.47 \pm 0.19$ & $6.04 \pm 0.01$ \\
\hline 16852 & 22484 & $10 \mathrm{Tau}$ & F9V & F9IV-V, F9V, F9IV-V & 033652.383 & +002405.98 & $71.62 \pm 0.54$ & $13.96 \pm 0.11$ \\
\hline 22449 & 30652 & 1 Ori & F6V & F6V, F6V-F6IV-V & 044950.411 & +065740.59 & $123.94 \pm 0.17$ & $8.07 \pm 0.01$ \\
\hline 23693 & 33262 & $\zeta$ Dor & F7V & F9V, F67/V-F8V & 050530.657 & -572821.74 & $85.87 \pm 0.18$ & $11.65 \pm 0.02$ \\
\hline 27072 & 38393 & $\gamma$ Lep & F7V & F6.5V, F6V-F8IV & 054427.791 & -222654.18 & $112.02 \pm 0.18$ & $8.93 \pm 0.01$ \\
\hline 37279 & 61421 & $\alpha \mathrm{CMi} \mathrm{A}$ & F5IV-V & F5IV-V, F4V-F5IV-V & 073918.120 & +051329.96 & $284.56 \pm 1.26$ & $3.51 \pm 0.02$ \\
\hline 47080 & 82885 & $11 \mathrm{LMi}$ & G8IV-V & G8IIIv, G8V-G8IV & 093539.503 & +354836.49 & $87.96 \pm 0.32$ & $11.37 \pm 0.04$ \\
\hline 56997 & 101501 & $61 \mathrm{UMa}$ & G8Vvar & G8V & 114103.016 & +341205.88 & $104.04 \pm 0.26$ & $9.61 \pm 0.02$ \\
\hline 57443 & 102365 & LHS 311 & G3/G5V & G2V,G2.5III, G5V & 114631.073 & -403001.27 & $108.45 \pm 0.22$ & $9.22 \pm 0.02$ \\
\hline 57757 & 102870 & $\beta$ Vir & F8V & F9V, F8.5IV-V & 115041.718 & +014552.99 & $91.50 \pm 0.22$ & $10.93 \pm 0.03$ \\
\hline 59199 & 105452 & $\alpha \mathrm{Crv}$ & FOIV/V & F1V, F0IV-V, F2III-IV, F2V & 120824.817 & -244343.95 & $66.95 \pm 0.15$ & $14.94 \pm 0.03$ \\
\hline 61317 & 109358 & $\beta \mathrm{CVn}$ & G0V & G0V & 123344.545 & +412126.92 & $118.49 \pm 0.20$ & $8.44 \pm 0.01$ \\
\hline 61941 & $110379 \mathrm{~J}$ & $29 \mathrm{Vir}$ & $\mathrm{F} 0 \mathrm{~V}+\ldots$ & F0V, A7V-F2V & 124139.643 & -012657.74 & $85.58 \pm 0.60$ & $11.68 \pm 0.08$ \\
\hline 64394 & 114710 & $\beta$ Com & G0V & $\mathrm{G} 0 \mathrm{~V}, \mathrm{~F} 8 / 9 \mathrm{~V}-\mathrm{G} 0 \mathrm{~V}$ & 131152.394 & +275241.45 & $109.54 \pm 0.17$ & $9.13 \pm 0.01$ \\
\hline 64924 & 115617 & $61 \mathrm{Vir}$ & G5V & G7V, G6.5V & 131824.314 & -181840.30 & $116.89 \pm 0.22$ & $8.56 \pm 0.02$ \\
\hline 70497 & 126660 & $\theta$ Boo & F7V & F7V, F7II-III & 142511.797 & +515102.68 & $68.82 \pm 0.14$ & $14.53 \pm 0.03$ \\
\hline 72659 & 131156 & 37 Boo & $\mathrm{G} 8 \mathrm{~V}+\mathrm{K} 4 \mathrm{~V}$ & G8V, G7V-G8V & 145123.380 & +190601.70 & $148.98 \pm 0.48$ & $6.71 \pm 0.02$ \\
\hline 73695 & 133640 & 44 Boo & $\mathrm{G} 2 \mathrm{~V}+\mathrm{G} 2 \mathrm{~V}$ & G0Vnv, F5V-G2V & 150347.296 & +473914.62 & $79.95 \pm 1.56$ & $12.51 \pm 0.24$ \\
\hline 77257 & 141004 & $\lambda \mathrm{Ser}$ & G0Vvar & G0V, G0IV-V-G0V & 154626.614 & +072111.04 & $82.48 \pm 0.32$ & $12.12 \pm 0.05$ \\
\hline 78072 & 142860 & $\gamma$ Ser & F6V & F6IV, F6V-F7V & 155627.183 & +153941.82 & $88.86 \pm 0.18$ & $11.25 \pm 0.02$ \\
\hline 80686 & 147584 & $\zeta \operatorname{TrA}$ & F9V & F9V, F6(V)+G1?(V) & 162828.144 & -700503.84 & $82.53 \pm 0.52$ & $12.12 \pm 0.08$ \\
\hline 89937 & 170153 & $\chi$ Dra & F7Vvar & F7V, F7V-F8V & 182103.383 & +724358.25 & $124.11 \pm 0.87$ & $8.06 \pm 0.06$ \\
\hline 93017 & 176051 & HR 7162 & G0V & F9V, F9V-G0V & 185701.610 & +325404.57 & $67.24 \pm 0.37$ & $14.87 \pm 0.08$ \\
\hline 99825 & 192310 & HR 7722 & $\mathrm{~K} 3 \mathrm{~V}$ & $\mathrm{~K} 2+\mathrm{v}, \mathrm{K} 2 \mathrm{~V}$ & 201517.391 & -270158.71 & $112.22 \pm 0.30$ & $8.91 \pm 0.02$ \\
\hline 102485 & 197692 & $\psi$ Cap & F5V & F5V, F4V-F6V & 204605.733 & -251615.23 & $68.13 \pm 0.27$ & $14.68 \pm 0.06$ \\
\hline 105858 & 203608 & $\gamma \mathrm{Pav}$ & F6V & F9V, F6V-F8V & 212626.605 & -652158.31 & $107.97 \pm 0.19$ & $9.26 \pm 0.02$ \\
\hline 109176 & 210027 & $\iota$ Peg & F5V & F5V, F5V-F6V & 220700.666 & +252042.40 & $85.28 \pm 0.63$ & $11.73 \pm 0.09$ \\
\hline 113283 & 216803 & TW PsA & $\mathrm{K} 4 \mathrm{Vp}$ & $\mathrm{K} 4+\mathrm{vk}, \mathrm{K} 3 \mathrm{~V}-\mathrm{K} 5 \mathrm{~V}$ & 225624.053 & -313356.04 & $131.42 \pm 0.62$ & $7.61 \pm 0.04$ \\
\hline 116771 & 222368 & $\iota \mathrm{Psc}$ & F7V & F7V, F7V-F8V & 233957.041 & +053734.65 & $72.92 \pm 0.15$ & $13.71 \pm 0.03$ \\
\hline \multicolumn{9}{|c|}{ FGK stars $(15 \mathrm{pc}<d \leq 20 \mathrm{pc})$} \\
\hline 5862 & 7570 & $v$ Phe & F8V & F9V, F9V & 011511.121 & -453154.00 & $66.16 \pm 0.24$ & $15.11 \pm 0.05$ \\
\hline 17651 & 23754 & 27 Eri & F3/F5V & F5IV-V, F3III & 034650.888 & -231459.00 & $56.73 \pm 0.19$ & $17.63 \pm 0.06$ \\
\hline 36366 & 58946 & $\rho \mathrm{Gem}$ & F0V... & F0V, F0V-F3V & 072906.719 & +314704.38 & $55.41 \pm 0.24$ & $18.05 \pm 0.08$ \\
\hline 44248 & 76943 & $10 \mathrm{UMa}$ & F5V & F5V, F5V-F7V & 090038.381 & +414658.61 & $62.23 \pm 0.68$ & $16.07 \pm 0.18$ \\
\hline 46509 & 81997 & 31 Hya & F6V & F6V, F5.5IV-V-F6IV-V & 092908.897 & -024608.26 & $57.69 \pm 2.14$ & $17.33 \pm 0.64$ \\
\hline 48113 & 84737 & $15 \mathrm{LMi}$ & $\mathrm{G} 2 \mathrm{~V}$ & G0.5Va, G0IV-V & 094835.371 & +460115.63 & $54.44 \pm 0.28$ & $18.37 \pm 0.09$ \\
\hline 61174 & 109085 & $\eta \mathrm{Crv}$ & $\mathrm{F} 2 \mathrm{~V}$ & F2V, FOIII, FOIV, F2III & 123204.227 & -161145.62 & $54.70 \pm 0.17$ & $18.28 \pm 0.06$ \\
\hline 64241 & $114378 \mathrm{~J}$ & 42 Com & F5V & F5V, F5.5V-F7V & 130959.285 & +173146.04 & $56.10 \pm 0.89$ & $17.83 \pm 0.28$ \\
\hline 67153 & 119756 & i Cen & F3V & F2V, F3IV, F2V & 134541.245 & -330237.40 & $51.54 \pm 0.19$ & $19.40 \pm 0.07$ \\
\hline 71284 & 128167 & $\sigma$ Boo & F3Vwvar & F2V, F3V-F5V & 143440.817 & +294442.46 & $63.16 \pm 0.25$ & $15.83 \pm 0.06$ \\
\hline 75312 & $137107 \mathrm{~J}$ & $\eta \mathrm{CrB}$ & $\mathrm{G} 2 \mathrm{~V}$ & $\mathrm{G} 2 \mathrm{~V}, \mathrm{~F} 9 \mathrm{~V}-\mathrm{G} 2 \mathrm{~V}$ & 152312.305 & +301716.17 & $55.98 \pm 0.78$ & $17.86 \pm 0.25$ \\
\hline 77760 & 142373 & $\chi$ Her & F9V & F8Ve..., F8V-G0V & 155240.541 & +422705.47 & $62.92 \pm 0.21$ & $15.89 \pm 0.05$ \\
\hline 88745 & 165908 & 99 Her A & F7V & F7V, F7V-F9V & 180701.540 & +303343.69 & $63.93 \pm 0.34$ & $15.64 \pm 0.08$ \\
\hline 104858 & 202275 & $\delta$ Equ & $\mathrm{F} 5 \mathrm{~V}+\ldots$ & $\mathrm{F} 5 \mathrm{~V}+\ldots, \mathrm{F} 7 \mathrm{~V}-\mathrm{G} 1 \mathrm{~V}$ & 211428.815 & +100025.13 & $54.09 \pm 0.66$ & $18.49 \pm 0.23$ \\
\hline 112447 & 215648 & $\xi$ Peg & F7V & F7V, F5V-F7V & 224641.581 & +121022.39 & $61.36 \pm 0.19$ & $16.30 \pm 0.05$ \\
\hline
\end{tabular}


A\&A 593, A51 (2016)

Table C.2. Parameters of the DUNES_DB stars with $d \leq 15 \mathrm{pc}$ and $15 \mathrm{pc}<d \leq 20 \mathrm{pc}$.

\begin{tabular}{|c|c|c|c|c|c|c|c|c|c|c|}
\hline HIP & $\begin{array}{l}T_{\text {eff }} \\
(\mathrm{K})\end{array}$ & $\begin{array}{c}\log g \\
\left(\mathrm{~cm} / \mathrm{s}^{2}\right)\end{array}$ & $\begin{array}{c}{[\mathrm{Fe} / \mathrm{H}]} \\
(\mathrm{dex})\end{array}$ & Flag & $\begin{array}{l}L_{\text {bol }} \\
\left(L_{\odot}\right) \\
\end{array}$ & $\log R_{\mathrm{HK}}^{\prime}$ & Ref. & $\begin{array}{c}P_{\text {rot }}(\mathrm{d}) \\
\text { or lower limit } \dagger\end{array}$ & $\begin{array}{l}t_{\text {Gyro }} \\
(\mathrm{Gyr}) \\
\end{array}$ & $\begin{array}{c}t_{\mathrm{HK}} \\
(\mathrm{Gyr}) \\
\end{array}$ \\
\hline \multicolumn{11}{|c|}{ FGK stars $(d \leq 15 \mathrm{pc})$} \\
\hline 1599 & 5960 & 4.45 & -0.17 & $S$ & 1.224 & -4.855 & 1 & $14.01 \pm 0.97$ & 2.20 & 4.03 \\
\hline 3765 & 4977 & 4.57 & -0.22 & $\mathrm{~S}$ & 0.283 & -4.852 & 2 & $39.02 \pm 0.25$ & 5.41 & 3.98 \\
\hline 7751 & 4993 & 4.54 & -0.23 & S & 0.304 & -4.94 & 3 & & & \\
\hline 7918 & 5891 & 4.36 & +0.06 & S & 1.405 & -4.987 & 4 & $21.10 \pm 0.12$ & 3.56 & 6.36 \\
\hline 7981 & 5189 & 4.52 & -0.04 & $\mathrm{~S}$ & 0.439 & -4.912 & 2 & $39.60 \pm 0.43$ & 6.04 & 4.99 \\
\hline 10644 & 5684 & 4.36 & -0.45 & $\mathrm{P}$ & 1.118 & -4.644 & 4 & $11.57 \pm 0.35$ & 1.30 & 1.47 \\
\hline 12114 & 4866 & 4.66 & +0.00 & $\mathrm{~S}$ & 0.257 & -4.958 & 2 & $44.90 \pm 0.53$ & 6.66 & 5.82 \\
\hline 12843 & 6273 & 4.20 & -0.04 & $P$ & 2.585 & -4.524 & 1 & $2.92 \pm 0.36$ & & 0.71 \\
\hline 14879 & 6275 & 4.40 & -0.19 & $\mathrm{~S}$ & 4.923 & -4.901 & 1 & $11.58 \pm 1.39$ & 2.12 & 4.79 \\
\hline 15457 & 5755 & 4.52 & +0.11 & $\mathrm{~S}$ & 0.838 & -4.42 & 2 & $7.53 \pm 0.18$ & 0.46 & 0.35 \\
\hline 15510 & 5415 & 4.55 & -0.34 & $S$ & 0.637 & -4.998 & 1 & $32.42 \pm 0.55$ & 5.51 & 6.56 \\
\hline 16852 & 6014 & 4.08 & -0.06 & $\mathrm{~S}$ & 3.060 & -5.053 & 4 & $17.06 \pm 0.95$ & 3.13 & 7.59 \\
\hline 22449 & 6457 & 4.15 & -0.01 & $\mathrm{~S}$ & 2.708 & -4.788 & 4 & $5.83 \pm 0.18$ & & 3.04 \\
\hline 23693 & 6165 & 4.38 & -0.20 & $P$ & 1.414 & -4.49 & 3 & $3.91 \pm 0.37$ & 0.40 & 0.57 \\
\hline 27072 & 6331 & 4.28 & -0.10 & $\mathrm{P}$ & 2.291 & -4.817 & 1 & $5.91 \pm 0.66$ & & 3.45 \\
\hline 37279 & 6578 & 3.99 & +0.01 & $\mathrm{~S}$ & 6.605 & -4.777 & 2 & $3.21 \pm 0.59$ & & 2.89 \\
\hline 47080 & 5539 & 4.48 & +0.33 & $\mathrm{~S}$ & 0.822 & -4.638 & 2 & $21.86 \pm 0.10$ & 2.39 & 1.42 \\
\hline 56997 & 5514 & 4.50 & -0.04 & $\mathrm{~S}$ & 0.618 & -4.546 & 2 & $14.62 \pm 0.65$ & 1.31 & 0.82 \\
\hline 57443 & 5630 & 4.51 & -0.28 & S & 0.806 & -4.957 & 1 & $25.90 \pm 0.47$ & 4.27 & 5.80 \\
\hline 57757 & 6125 & 4.12 & +0.14 & $\mathrm{~S}$ & 3.483 & -4.99 & 5 & $10.27 \pm 1.37$ & 2.62 & 6.41 \\
\hline 59199 & 7042 & 4.21 & -0.14 & $P$ & 4.175 & -5.373 & 6 & $>3.26(21.7)$ & & \\
\hline 61317 & 5890 & 4.40 & -0.17 & $S$ & 1.178 & -4.851 & 4 & $15.11 \pm 0.89$ & 2.32 & 3.96 \\
\hline 61941 & 6797 & 4.25 & -0.13 & $\mathrm{P}$ & 8.346 & -4.50 & 6 & $6.31 \pm 0.37 \ddagger$ & & 0.61 \\
\hline 64394 & 6065 & 4.43 & +0.04 & $\mathrm{~S}$ & 1.381 & -4.745 & 2 & $11.48 \pm 0.49$ & 1.59 & 2.49 \\
\hline 64924 & 5646 & 4.57 & +0.10 & $S$ & 0.820 & -5.001 & 2 & $32.29 \pm 0.78$ & 5.50 & 6.62 \\
\hline 70497 & 6315 & 4.29 & -0.10 & $\mathrm{P}$ & 4.034 & -4.591 & 4 & $4.26 \pm 0.21$ & 2.25 & 1.08 \\
\hline 72659 & 5549 & 4.63 & -0.10 & $\mathrm{~S}$ & 0.611 & -4.363 & 2 & $6.49 \pm 0.31$ & 0.31 & 0.23 \\
\hline 73695 & 5851 & 4.30 & -0.19 & $S$ & 1.552 & -4.602 & 4 & $12.85 \pm 0.13$ & 1.31 & 1.16 \\
\hline 77257 & 5899 & 4.17 & +0.01 & $\mathrm{~S}$ & 2.077 & -5.004 & 2 & $19.73 \pm 0.96$ & 3.40 & 6.67 \\
\hline 78072 & 6265 & 4.08 & -0.15 & S & 2.863 & -5.113 & 4 & $>5.2(11.42)$ & & \\
\hline 80686 & 6029 & 4.46 & -0.19 & $\mathrm{P}$ & 1.301 & -4.65 & 3 & $8.25 \pm 1.00$ & 1.02 & 1.52 \\
\hline 89937 & 6034 & 4.27 & -0.65 & $\mathrm{P}$ & 1.942 & -4.9 & 6 & $7.13 \pm 0.35$ & & 4.78 \\
\hline 93017 & 5834 & 4.33 & -0.19 & $\mathrm{P}$ & 1.515 & -4.874 & 2 & $16.18 \pm 0.31$ & 2.53 & 4.34 \\
\hline 99825 & 5105 & 4.48 & -0.03 & $\mathrm{~S}$ & 0.405 & -5.048 & 1 & $47.33 \pm 0.78$ & 7.74 & 7.50 \\
\hline 102485 & 6590 & 4.26 & -0.05 & $\mathrm{P}$ & 3.705 & -4.404 & 1 & $0.94 \pm 0.06$ & & 0.31 \\
\hline 105858 & 6089 & 4.38 & -0.68 & $\mathrm{P}$ & 1.400 & -4.491 & 1 & $2.93 \pm 0.57$ & & 0.57 \\
\hline 109176 & 6448 & 4.25 & 0.12 & $\mathrm{P}$ & 3.303 & -5.06 & 7 & $4.61 \pm 0.39$ & & 7.72 \\
\hline 113283 & 4555 & 4.53 & -0.01 & $\mathrm{~S}$ & 0.181 & -4.467 & 1 & $16.03 \pm 0.03$ & 0.88 & 0.49 \\
\hline 116771 & 6180 & 4.09 & -0.10 & $\mathrm{~S}$ & 3.319 & -5.112 & 4 & $>8.58(6.67)$ & $>2.27$ & \\
\hline \multicolumn{11}{|c|}{ FGK stars $(15 \mathrm{pc}<d \leq 20 \mathrm{pc})$} \\
\hline 5862 & 6128 & 4.36 & +0.16 & S & 1.913 & -4.861 & 1 & $13.63 \pm 0.68$ & 2.17 & 4.12 \\
\hline 17651 & 6654 & 4.11 & +0.09 & $\mathrm{P}$ & 4.930 & -4.684 & 1 & $2.74 \pm 0.40$ & & 1.83 \\
\hline 36366 & 7086 & 4.13 & -0.25 & $\mathrm{P}$ & 5.351 & -4.66 & 6 & $2.50 \pm 0.36^{\ddagger}$ & & 1.61 \\
\hline 44248 & 6556 & 4.01 & +0.04 & $\mathrm{P}$ & 5.249 & -4.548 & 8 & $2.62 \pm 0.09$ & & 0.83 \\
\hline 46509 & 6486 & 4.22 & -0.01 & $\mathrm{P}$ & 3.369 & -4.828 & 4 & $2.66 \pm 0.52$ & & 3.61 \\
\hline 48113 & 5940 & 4.05 & +0.14 & $\mathrm{~S}$ & 2.618 & -5.042 & 4 & $22.30 \pm 0.89$ & 3.91 & 7.39 \\
\hline 61174 & 6784 & 4.06 & -0.05 & $\mathrm{P}$ & 4.873 & -5.173 & 6 & $>1.12(59.15)$ & & \\
\hline 64241 & 6376 & 4.26 & -0.21 & $\mathrm{P}$ & 4.625 & -4.53 & 2 & $2.26 \pm 0.20$ & & 0.74 \\
\hline 67153 & 6745 & 3.88 & -0.09 & $\mathrm{P}$ & 5.857 & -4.56 & 6 & $2.42 \pm 0.22^{\ddagger}$ & & 0.90 \\
\hline 71284 & 6727 & 4.32 & -0.35 & $\mathrm{~S}$ & 3.112 & -5.476 & 4 & $>9.34(7.32)$ & & \\
\hline 75312 & 5949 & 4.15 & -0.06 & $\mathrm{~S}$ & 2.631 & -4.828 & 2 & $13.60 \pm 0.38$ & 2.07 & 3.61 \\
\hline 77760 & 5840 & 3.94 & -0.49 & $\mathrm{~S}$ & 2.962 & -5.042 & 2 & $15.50 \pm 1.59$ & 2.91 & 7.39 \\
\hline 88745 & 6089 & 4.18 & -0.53 & $\mathrm{~S}$ & 1.867 & -5.02 & 9 & $11.52 \pm 0.20$ & 2.60 & 6.98 \\
\hline 104858 & 6250 & 4.40 & -0.07 & $\mathrm{P}$ & 4.450 & -4.905 & 8 & $10.36 \pm 1.32$ & 2.12 & 4.86 \\
\hline 112447 & 6181 & 3.93 & -0.24 & $S$ & 4.387 & -5.276 & 4 & $>4.55(12.67)$ & $>1.23$ & \\
\hline
\end{tabular}

Notes. $T_{\text {eff }}, \log g$, and $[\mathrm{Fe} / \mathrm{H}]$ are averages from Fuhrmann (2008), Gray et al. (2003), Gray et al. (2006), Holmberg et al. (2009), Santos et al. (2004), Sousa et al. (2008), Takeda et al. (2005), and Valenti \& Fischer (2005). "S" and "P" in Col. 5 mean that the determination is spectroscopic or photometric. $\log g$ for HIP 104858 assigned according to its spectral type.

References. $\log R_{\mathrm{HK}}^{\prime}$ : (1) Gray et al. (2006); (2) Baliunas et al. (1996); (3) Martínez-Arnáiz et al. (2010); (4) Duncan et al. (1991); (5) Hall et al. (2007); (6) $R_{\mathrm{HK}}^{\prime}$ computed from ROSAT X-ray data using Eq. (A1) from Mamajek \& Hillenbrand (2008); (7) Saffe et al. (2005); (8) Gray et al. (2003); (9) Wright et al. (2004). Determination of $P_{\text {rot }}$ from the Rossby number and $\log R_{\mathrm{HK}}^{\prime}$ valid if $0.4<B-V<1.4$ and $-5.1<\log R_{\mathrm{HK}}^{\prime}<-4.3$ (Noyes et al. 1984). ${ }^{(\dagger)}$ When a lower limit of the period is given, the number in parentheses is the value of $v \sin i\left(\mathrm{~km} \mathrm{~s}^{-1}\right)$ used to estimate it. $\left.{ }^{(\ddagger)}\right)$ The $(B-V)$ value is slightly outside the valid range of the Noyes et al. (1984) calibration for $P_{\text {rot }}$. Gyrochronology age calibration valid if $B-V>0.5$ (Mamajek \& Hillenbrand 2008). Chromospheric age calibration valid in the interval $-5.0<\log R_{\mathrm{HK}}^{\prime}<-4.3$ (Mamajek \& Hillenbrand 2008). 
B. Montesinos et al.: Debris discs around nearby FGK main-sequence stars

Table C.3. Multiplicity and planetary companions of the DUNES_DB stars with $d \leq 20$ pc.

\begin{tabular}{|c|c|c|c|c|c|c|c|}
\hline \multirow[t]{2}{*}{ HIP } & \multirow{2}{*}{$\begin{array}{l}\text { Multiplicity } \\
\text { and details }\end{array}$} & \multirow[t]{2}{*}{ CCDM identification } & \multicolumn{2}{|c|}{ ICRS (2000) } & \multirow{2}{*}{$\begin{array}{r}\mu_{\alpha} \cos (\delta) \\
(\mathrm{mas} / \mathrm{yr})\end{array}$} & \multirow{2}{*}{$\begin{array}{r}\mu_{\delta} \\
(\mathrm{mas} / \mathrm{yr})\end{array}$} & \multirow{2}{*}{$\begin{array}{l}\text { Separation } \\
(\operatorname{arcsec})\end{array}$} \\
\hline & & & $\alpha$ (hh mm ss) & $\delta($ deg $\min \mathrm{sec})$ & & & \\
\hline \multicolumn{8}{|c|}{ FGK stars $(d \leq 15 \mathrm{pc})$} \\
\hline 7751 & Double or multiple star & & & & & & \multirow{3}{*}{11.18} \\
\hline & p Eri B K2V $V=5.797$ & CCDM J01398-5612A & 013947.557 & -561147.34 & 286.10 & 16.16 & \\
\hline & p Eri A K 2 V $V=5.96$ & CCDM J01398-5612B & 013947.555 & -561136.16 & 286.10 & 16.16 & \\
\hline 7918 & Spectroscopic binary & & & & & & \\
\hline 10644 & Spectroscopic binary & CCDM J02170+3414A & & & & & \\
\hline 12843 & Spectroscopic binary & & & & & & \\
\hline \multirow[t]{3}{*}{14879} & Double or multiple star & & & & & & \multirow{3}{*}{7.50} \\
\hline & $\alpha$ For A, F6V, $V=3.98$ & CCDM J03121-2859A & 031204.294 & -285920.42 & 371.49 & 612.26 & \\
\hline & $\alpha$ For B, G7V, $V=7.1$ & CCDM J03121-2859B & 031204.211 & -285913.00 & 345.78 & 648.31 & \\
\hline \multirow[t]{3}{*}{37279} & Spectroscopic binary & & & & & & \multirow{3}{*}{0.27} \\
\hline & $\alpha$ CMi A, F5IV-V, $V=0.37$ & CCDM J07393+0514A & 073918.118 & +051329.97 & & & \\
\hline & $\alpha \mathrm{CMi} \mathrm{B}, \mathrm{DQZ}, V=10.92$ & CCDM J07393+0514B & 073918.1 & +051330 & & & \\
\hline \multirow[t]{3}{*}{47080} & Variable of RS CVn type & & & & & & \multirow{3}{*}{6.11} \\
\hline & 11 LMi A, G8+V, $V=5.48$ & CCDM J09357+3549A & 093539.506 & +354836.75 & & & \\
\hline & $11 \mathrm{LMi} \mathrm{B}, \mathrm{M} 5 \mathrm{~V}, V=13.0$ & CCDM J09357+3549B & 093539.97 & +354839.1 & & & \\
\hline 61317 & Spectroscopic binary & CCDM J12337+4121AB & & & & & \\
\hline \multirow[t]{3}{*}{61941} & Double or multiple star & & & & & & \multirow{3}{*}{2.62} \\
\hline & $\gamma$ Vir A, F0IV, $V=3.440$ & CCDM J12417-0127A & 124139.983 & -012658.25 & -616.66 & +60.66 & \\
\hline & $\gamma$ Vir B, FOIV, $V=3.484$ & CCDM J12417-0127B & 124139.813 & -012657.63 & -523.91 & -39.05 & \\
\hline 72659 & Variable of BY Dra type & & & & & & \\
\hline & $\xi$ Boo A, G7Ve, $V=4.675$ & CCDM J14513+1906A & 145123.286 & +190602.25 & 152.81 & -71.28 & \\
\hline & $\xi$ Boo B, K5Ve, $V=6.816$ & CCDM J14513+1906B & & & 89.72 & -147.30 & \\
\hline 73695 & $\begin{array}{l}\text { Eclipsing binary of W UMa } \\
\text { type (contact binary) }\end{array}$ & & & & & & \\
\hline & i Boo A, F5V, $V=5.136$ & CCDM J15038+4739A & 150347.301 & +473914.55 & -436.24 & 18.94 & \\
\hline & i Boo B, G9:, $V=6.004$ & CCDM J15038+4739B & 150347.484 & +473915.93 & -378.67 & 40.02 & 2.31 \\
\hline 77257 & Spectroscopic binary & & & & & & \\
\hline 80686 & Spectroscopic binary & & & & & & \\
\hline 89937 & Spectroscopic binary & CCDM J18211+7245A & & & & & \\
\hline 93017 & Spectroscopic binary & & & & & & \\
\hline & HD $176051 \mathrm{~A}, \mathrm{~F} 9 \mathrm{~V}, V=5.277$ & CCDM J18570+3254A & 185701.470 & +325405.77 & 202.85 & -143.97 & 0.99 \\
\hline & HD 176051B, K1V, V 7.84 & CCDM J18570+3254B & 185701.493 & +325406.72 & 124.14 & -137.92 & 0.99 \\
\hline 109176 & Spectroscopic binary & CCDM J22070+2520A & & & & & \\
\hline FGK sta & $(15 \mathrm{pc}<d \leq 20 \mathrm{pc})$ & & & & & & \\
\hline 36366 & Double or multiple star & & & & & & \\
\hline & $\rho \mathrm{Gem}, \mathrm{F} 1 \mathrm{~V}, V=4.18$ & CCDM J07290+3147AB & 072906.71887 & +314704.3773 & & & 1242 \\
\hline & $\rho$ Gem B, M5 D, $V=12.5$ & CCDM J07290+3147B & 072906.0 & +314656 & & & 12.42 \\
\hline 44248 & Spectroscopic binary & & & & & & \\
\hline & $10 \mathrm{UMa} \mathrm{A}, \mathrm{F} 3 \mathrm{~V}, V=4.14$ & CCDM J09007+4147A & 090038.38 & +414658.6 & -487.67 & -219.29 & 0.00 \\
\hline & $10 \mathrm{UMa} B, \mathrm{G} 5 \mathrm{~V}, V=5.97$ & CCDM J09007+4147B & 090038.38 & +414658.6 & -381.62 & -270.08 & 0.00 \\
\hline 46509 & Spectroscopic binary & & & & & & \\
\hline & $\tau^{01}$ Hya, F6V, $V=4.60$ & CCDM J09291-0246A & 092908.89655 & -024608.2649 & & & 6556 \\
\hline & $\tau^{01}$ Hya B, K0 D, $V=7.15$ & CCDM J09291-0246B & 092909.2290 & -024502.897 & & & 03.56 \\
\hline 64241 & Double or multiple star & & & & & & \\
\hline & $\alpha$ Com A, F5V, $V=4.85$ & CCDM J13100+1732A & 130959.28 & +173145.86 & & & 038 \\
\hline & $\alpha$ Com B, F6V, $V=5.53$ & CCDM J13100+1732B & 130959.28 & +173146.24 & & & 0.30 \\
\hline 67153 & Spectroscopic binary & & & & & & \\
\hline 75312 & Spectroscopic binary & & & & & & \\
\hline & $\eta \mathrm{CrB} \mathrm{A}, \mathrm{G} 2 \mathrm{~V}, V=5.577$ & CCDM J15233+3018A & 152312.23 & +301717.56 & 125.77 & -176.48 & 103 \\
\hline & $\eta \mathrm{CrB} \mathrm{B}, \mathrm{G} 2 \mathrm{~V}, V=5.95$ & CCDM J15233+3018B & 152312.27 & +301718.46 & 169.99 & -204.58 & 1.03 \\
\hline 88745 & Double or multiple star & & & & & & \\
\hline & b Her A, F7V, $V=5.13$ & CCDM J18071+3034A & 180701.60 & +303342.77 & -100.93 & 111.70 & 127 \\
\hline & b Her B, K4V, $V=8.96$ & CCDM J18071+3034B & 180701.59 & +303344.03 & -69.00 & 48.08 & 1.21 \\
\hline 104858 & Spectroscopic binary & CCDM J21145+1001AB & & & 42.32 & -303.43 & \\
\hline
\end{tabular}

\begin{tabular}{|c|c|c|c|c|c|}
\hline \multirow[t]{2}{*}{ HIP } & \multirow[t]{2}{*}{ Comment } & \multirow[t]{2}{*}{ CCDM identification } & \multicolumn{3}{|c|}{ Planets } \\
\hline & & & & $M / M_{\text {Jup }}$ & a (UA) \\
\hline \multirow[t]{3}{*}{15510} & High proper-motion star & & $\mathrm{b}$ & 0.009 & 0.12 \\
\hline & & & $\mathrm{c}$ & 0.008 & 0.20 \\
\hline & & & d & 0.015 & 0.35 \\
\hline 57443 & High proper-motion star & CCDM J11465-4030A & $\mathrm{b}$ & 0.05 & 0.46 \\
\hline \multirow{3}{*}{64924} & High proper-motion star & CCDM J13185-1818A & $\mathrm{b}$ & 0.016 & 0.05 \\
\hline & & & $\mathrm{c}$ & 0.057 & 0.22 \\
\hline & & & $\mathrm{d}$ & 0.072 & 0.48 \\
\hline 93017 & Spectroscopic binary & & $\mathrm{b}$ & 1.5 & 1.76 \\
\hline \multirow[t]{2}{*}{99825} & Variable star & & $\mathrm{b}$ & 0.053 & 0.32 \\
\hline & & & $\mathrm{c}$ & 0.076 & 1.18 \\
\hline
\end{tabular}

Notes. All planets were discovered by the radial velocity (RV) method except HIP 93017b (astrometry); therefore, masses for the RV exoplanets are lower limits. Data from http: //exoplanetarchive.ipac. caltech. edu/docs/data.html except for HIP 93017b, which are taken from http://exoplanet.eu 
A\&A 593, A51 (2016)

Table C.4. DUNES_DB stars with $d \leq 20$ pc: Johnson $V, B-V$, Cousins $V-I$, and Strömgren photometry.

\begin{tabular}{|c|c|c|c|c|c|c|}
\hline HIP & $\begin{array}{c}V \\
(\mathrm{mag})\end{array}$ & $\begin{array}{c}B-V \\
(\mathrm{mag})\end{array}$ & $\begin{array}{c}V-I \\
(\mathrm{mag})\end{array}$ & $\begin{array}{c}b-y \\
\text { (mag) }\end{array}$ & $\begin{array}{c}m_{1} \\
(\mathrm{mag})\end{array}$ & $\begin{array}{c}c_{1} \\
(\mathrm{mag})\end{array}$ \\
\hline \multicolumn{7}{|c|}{ FGK stars $(d \leq 15 \mathrm{pc})$} \\
\hline 1599 & 4.23 & $0.576 \pm 0.010$ & $0.65 \pm 0.02$ & $0.368 \pm 0.003$ & $0.177 \pm 0.004$ & $0.302 \pm 0.020$ \\
\hline 3765 & 5.74 & $0.890 \pm 0.008$ & $0.97 \pm 0.02$ & $0.512 \pm 0.003$ & $0.423 \pm 0.002$ & $0.255 \pm 0.004$ \\
\hline 7751 & 5.76 & $0.880 \pm 0.400$ & $0.93 \pm 0.02$ & 0.512 & 0.421 & 0.262 \\
\hline 7918 & 4.96 & $0.618 \pm 0.001$ & $0.67 \pm 0.03$ & $0.389 \pm 0.000$ & $0.198 \pm 0.005$ & $0.348 \pm 0.011$ \\
\hline 7981 & 5.24 & $0.836 \pm 0.008$ & $0.88 \pm 0.01$ & $0.492 \pm 0.002$ & $0.367 \pm 0.007$ & $0.296 \pm 0.006$ \\
\hline 10644 & 4.84 & $0.607 \pm 0.005$ & $0.76 \pm 0.07$ & $0.390 \pm 0.003$ & $0.186 \pm 0.003$ & $0.259 \pm 0.004$ \\
\hline 12114 & 5.79 & $0.918 \pm 0.019$ & $1.06 \pm 0.02$ & $0.555 \pm 0.003$ & $0.517 \pm 0.002$ & $0.273 \pm 0.006$ \\
\hline 12843 & 4.47 & $0.481 \pm 0.012$ & $0.54 \pm 0.02$ & 0.328 & 0.167 & 0.406 \\
\hline 14879 & 3.80 & $0.543 \pm 0.015$ & $0.63 \pm 0.02$ & 0.339 & 0.156 & 0.411 \\
\hline 15457 & 4.84 & $0.681 \pm 0.006$ & $0.73 \pm 0.02$ & $0.419 \pm 0.003$ & $0.235 \pm 0.005$ & $0.307 \pm 0.003$ \\
\hline 15510 & 4.26 & $0.711 \pm 0.005$ & $0.79 \pm 0.02$ & $0.440 \pm 0.004$ & $0.229 \pm 0.005$ & $0.295 \pm 0.009$ \\
\hline 16852 & 4.29 & $0.575 \pm 0.008$ & $0.66 \pm 0.02$ & $0.367 \pm 0.003$ & $0.173 \pm 0.004$ & $0.376 \pm 0.004$ \\
\hline 22449 & 3.19 & $0.484 \pm 0.003$ & $0.53 \pm 0.02$ & $0.298 \pm 0.002$ & $0.163 \pm 0.002$ & $0.415 \pm 0.004$ \\
\hline 23693 & 4.71 & $0.526 \pm 0.011$ & $0.60 \pm 0.00$ & $0.338 \pm 0.002$ & $0.167 \pm 0.004$ & $0.328 \pm 0.006$ \\
\hline 27072 & 3.59 & $0.481 \pm 0.011$ & $0.57 \pm 0.02$ & $0.314 \pm 0$ & $0.162 \pm 0.007$ & $0.392 \pm 0.008$ \\
\hline 37279 & 0.40 & $0.432 \pm 0.015$ & $0.49 \pm 0.02$ & $0.272 \pm 0.000$ & $0.167 \pm 0.000$ & $0.532 \pm 0.000$ \\
\hline 47080 & 5.40 & $0.770 \pm 0.002$ & $0.78 \pm 0.01$ & $0.473 \pm 0.002$ & $0.307 \pm 0.006$ & $0.370 \pm 0.004$ \\
\hline 56997 & 5.31 & $0.723 \pm 0.014$ & $0.78 \pm 0.04$ & $0.444 \pm 0.000$ & $0.271 \pm 0.005$ & $0.291 \pm 0.004$ \\
\hline 57443 & 4.89 & $0.664 \pm 0.004$ & $0.73 \pm 0.02$ & $0.410 \pm 0.004$ & $0.208 \pm 0$. & $0.278 \pm 0.001$ \\
\hline 57757 & 3.59 & $0.518 \pm 0.015$ & $0.61 \pm 0.02$ & $0.354 \pm 0.003$ & $0.187 \pm 0.003$ & $0.416 \pm 0.009$ \\
\hline 59199 & 4.02 & $0.334 \pm 0.015$ & $0.40 \pm 0.02$ & $0.213 \pm 0.004$ & $0.163 \pm 0.006$ & $0.577 \pm 0.007$ \\
\hline 61317 & 4.24 & $0.588 \pm 0.009$ & $0.67 \pm 0.01$ & $0.385 \pm 0.000$ & $0.182 \pm 0.001$ & $0.298 \pm 0.006$ \\
\hline 61941 & 2.74 & $0.368 \pm 0.017$ & $0.43 \pm 0.02$ & 0.195 & 0.168 & 0.714 \\
\hline 64394 & 4.23 & $0.572 \pm 0.006$ & $0.67 \pm 0.04$ & $0.368 \pm 0.002$ & $0.192 \pm 0.002$ & $0.338 \pm 0.003$ \\
\hline 64924 & 4.74 & $0.709 \pm 0.007$ & $0.75 \pm 0.02$ & $0.433 \pm 0.001$ & $0.255 \pm 0.002$ & $0.325 \pm 0.005$ \\
\hline 70497 & 4.04 & $0.497 \pm 0.005$ & $9 \pm 0.12$ & & 0.156 & 0.418 \\
\hline 72659 & 4.54 & $0.720 \pm 0.015$ & $0.82 \pm 0.03$ & 0.449 & 0.263 & 0.255 \\
\hline 73695 & 4.83 & $0.647 \pm 0.002$ & $0.71 \pm 0.00$ & $0.420 \pm$ & $0.202 \pm$ & $0.289 \pm 0.006$ \\
\hline 77257 & 4.42 & $0.604 \pm 0.008$ & $0.66 \pm 0.02$ & $0.383 \pm($ & $0.192 \pm 0$ & $0.364 \pm 0.004$ \\
\hline 78072 & 3.85 & $0.478 \pm 0.006$ & $0.54 \pm 0.02$ & $0.319 \pm 0.003$ & $0.150 \pm 0.002$ & $0.401 \pm 0.002$ \\
\hline 80686 & 4.90 & $0.555 \pm 0.016$ & $0.64 \pm 0.02$ & $0.351 \pm 0.006$ & $0.175 \pm 0.003$ & $0.321 \pm 0.007$ \\
\hline 89937 & 3.55 & $0.489 \pm 0.005$ & $0.62 \pm 0.04$ & $0.336 \pm 0.002$ & $0.145 \pm 0.004$ & $0.320 \pm 0.012$ \\
\hline 93017 & 5.20 & $0.594 \pm 0.003$ & $0.66 \pm 0.00$ & $0.387 \pm 0.001$ & $0.188 \pm 0.000$ & $0.319 \pm 0.002$ \\
\hline 99825 & 5.73 & $0.878 \pm 0.018$ & $0.93 \pm 0.02$ & $0.516 \pm 0.005$ & $0.442 \pm 0.001$ & $0.292 \pm 0.003$ \\
\hline 102485 & 4.13 & $0.426 \pm 0.005$ & $0.49 \pm 0.02$ & $0.276 \pm 0.002$ & $0.163 \pm 0.004$ & $0.466 \pm 0.007$ \\
\hline 105858 & 4.21 & $0.494 \pm 0.020$ & $0.61 \pm 0.02$ & $0.331 \pm 0.004$ & $0.124 \pm 0.004$ & $0.313 \pm 0.007$ \\
\hline 109176 & 3.77 & \pm 0.007 & \pm 0.03 & $4 \pm 0.005$ & $0.161 \pm 0.004$ & $0.446 \pm 0.001$ \\
\hline 113283 & 6.48 & $1.094 \pm$ & $1.20 \pm 0.02$ & $0.631=$ & $0648+0$ & $0.175 \pm 0.002$ \\
\hline 116771 & 4.13 & $0.507 \pm 0.006$ & $0.59 \pm 0.02$ & $0.330 \pm 0.002$ & $0.163 \pm 0.004$ & $0.399 \pm 0.003$ \\
\hline \multicolumn{7}{|c|}{ FGK stars $(15 \mathrm{pc}<d \leq 20 \mathrm{pc})$} \\
\hline 5862 & 4.97 & $0.571 \pm 0$ & & & & 0.3 \\
\hline 17651 & 4.22 & $0.434 \pm 0.0$ & 2 & 02 & $5 \pm 0.003$ & \pm 0.005 \\
\hline 36366 & 4.16 & $0.320 \pm 0.012$ & $0.40 \pm 0.03$ & $0.215 \pm 0.000$ & $0.155 \pm 0.001$ & $0.615 \pm 0.001$ \\
\hline 44248 & 3.96 & $0.463 \pm 0.003$ & $0.53 \pm 0.00$ & 0.286 & 0.173 & 0.499 \\
\hline 46509 & 4.59 & $0.411 \pm 0.015$ & $0.52 \pm 0.02$ & $0.296 \pm 0.001$ & $0.164 \pm 0.002$ & $0.451 \pm 0.003$ \\
\hline 48113 & 5.08 & $0.619 \pm 0.007$ & $0.68 \pm 0.03$ & $0.389 \pm 0.001$ & $0.203 \pm 0.001$ & $0.383 \pm 0.003$ \\
\hline 61174 & 4.30 & $0.388 \pm 0.014$ & $0.44 \pm 0.02$ & $0.244 \pm 0.003$ & $0.167 \pm 0.006$ & $0.543 \pm 0.006$ \\
\hline 64241 & 4.32 & $0.455 \pm 0.008$ & $0.53 \pm 0.01$ & $0.304 \pm 0.005$ & $0.151 \pm 0.005$ & $0.389 \pm 0.016$ \\
\hline 67153 & 4.23 & $0.390 \pm 0.017$ & $0.44 \pm 0.02$ & $0.246 \pm 0.002$ & $0.163 \pm 0.005$ & $0.550 \pm 0.007$ \\
\hline 71284 & 4.47 & $0.364 \pm 0.005$ & $0.41 \pm 0.03$ & $0.254 \pm 0.003$ & $0.134 \pm 0.004$ & $0.480 \pm 0.005$ \\
\hline 75312 & 4.99 & $0.577 \pm 0.004$ & $0.65 \pm 0.00$ & $0.367 \pm 0.002$ & $0.186 \pm 0.001$ & $0.338 \pm 0.003$ \\
\hline 77760 & 4.60 & $0.563 \pm 0.014$ & $0.63 \pm 0.02$ & $0.381 \pm 0.001$ & $0.146 \pm 0.005$ & $0.328 \pm 0.004$ \\
\hline 88745 & 5.05 & $0.528 \pm 0.002$ & $0.60 \pm 0.00$ & $0.356 \pm 0.002$ & $0.135 \pm 0.003$ & $0.322 \pm 0.005$ \\
\hline 104858 & 4.47 & $0.529 \pm 0.015$ & $0.57 \pm 0.02$ & $0.330 \pm 0.003$ & $0.162 \pm 0.005$ & $0.401 \pm 0.015$ \\
\hline 112447 & 4.20 & $0.502 \pm 0.007$ & $0.60 \pm 0.03$ & $0.331 \pm 0.002$ & $0.147 \pm 0.002$ & $0.407 \pm 0.001$ \\
\hline
\end{tabular}

Notes. Johnson $B V$ and Cousins $I$ magnitudes are from the HIPPARcos catalogue (I/239 in Vizier); the calibration from magnitudes to fluxes is carried out using the zero points by Bessell (1979). Strömgren $b-y, m_{1}$, and $c_{1}$ are taken from Hauck \& Mermilliod (1997) (catalogue II/215 in Vizier) and Hauck \& Mermilliod (1998); these indices were converted into uvby magnitudes, and then into fluxes using the zero points by Gray (1998). 
B. Montesinos et al.: Debris discs around nearby FGK main-sequence stars

Table C.5. DUNES_DB stars with $d \leq 20$ pc: 2 MASS $J H K_{\mathrm{s}}$ and ancillary Johnson $J H K L L^{\prime} M$ photometry.

\begin{tabular}{|c|c|c|c|c|c|c|c|c|c|c|c|}
\hline HIP & $\begin{array}{c}\text { 2MASS } J \\
(\mathrm{mag})\end{array}$ & $\begin{array}{l}\text { 2MASS } H \\
\text { (mag) }\end{array}$ & $\begin{array}{c}\text { 2MASS } K_{\mathrm{s}} \\
(\mathrm{mag})\end{array}$ & Qflag & $\begin{array}{c}J \\
(\mathrm{mag})\end{array}$ & $\begin{array}{c}H \\
(\mathrm{mag})\end{array}$ & $\begin{array}{c}K \\
(\mathrm{mag}) \\
\end{array}$ & $\begin{array}{c}L \\
\text { (mag) }\end{array}$ & $\begin{array}{c}L^{\prime} \\
(\mathrm{mag})\end{array}$ & $\begin{array}{c}M \\
(\mathrm{mag})\end{array}$ & Refs. \\
\hline \multicolumn{12}{|c|}{ FGK stars $(d \leq 15 \mathrm{pc})$} \\
\hline 1599 & $3.068 \pm 0.272$ & $2.738 \pm 0.218$ & $2.769 \pm 0.250$ & DDD & 3.196 & 2.880 & 2.832 & 2.803 & 2.795 & 2.856 & $1,2,3$ \\
\hline 3765 & $4.367 \pm 0.310$ & $3.722 \pm 0.230$ & $3.683 \pm 0.268$ & DDD & & & & & & & \\
\hline $7751 \mathrm{~A}$ & & & $3.558 \pm 0.270$ & D & & & & & & & \\
\hline $7751 \mathrm{~B}$ & $4.043 \pm 0.378$ & & $3.510 \pm 0.282$ & D D & & & & & & & \\
\hline 7918 & $4.000 \pm 0.262$ & $3.703 \pm 0.226$ & $3.577 \pm 0.314$ & DDD & 3.800 & 3.560 & & & & & 2 \\
\hline 7981 & $3.855 \pm 0.240$ & $3.391 \pm 0.226$ & $3.285 \pm 0.266$ & DDD & 3.775 & 3.345 & 3.285 & & & & 2 \\
\hline 10644 & $3.525 \pm 0.246$ & $3.287 \pm 0.206$ & $3.076 \pm 0.276$ & DCD & 3.643 & 3.284 & 3.235 & 3.290 & & & 2,4 \\
\hline 12114 & $4.152 \pm 0.264$ & $3.657 \pm 0.244$ & $3.481 \pm 0.208$ & DDC & 4.068 & 3.543 & 3.457 & & & & 2 \\
\hline 12843 & $3.486 \pm 0.292$ & $3.236 \pm 0.306$ & $3.249 \pm 0.296$ & DDD & 3.607 & 3.380 & 3.339 & 3.350 & 3.308 & 3.328 & $2,3,5,6$ \\
\hline 14879 & $2.572 \pm 0.292$ & $2.317 \pm 0.268$ & $2.238 \pm 0.332$ & DDD & & & 2.510 & & & & 2 \\
\hline 15457 & $3.407 \pm 0.192$ & $3.039 \pm 0.182$ & $2.957 \pm 0.212$ & $\mathrm{CCC}$ & 3.668 & 3.353 & 3.288 & 3.259 & 3.190 & & $2,6,7$ \\
\hline 15510 & $3.032 \pm 0.262$ & $2.709 \pm 0.234$ & $2.636 \pm 0.278$ & DDD & 2.993 & 2.591 & 2.551 & 2.490 & & 2.560 & 1,3 \\
\hline 16852 & $3.194 \pm 0.246$ & $2.916 \pm 0.200$ & $2.835 \pm 0.260$ & DCD & 3.247 & & 2.911 & 2.905 & 2.840 & & $2,6,7$ \\
\hline 22449 & $1.984 \pm 0.246$ & $1.757 \pm 0.132$ & $1.600 \pm 0.196$ & DBC & 2.349 & 2.139 & 2.080 & 2.068 & 2.069 & 2.093 & $2,3,5,6,8,9$ \\
\hline 23693 & $3.701 \pm 0.248$ & $3.407 \pm 0.202$ & $3.371 \pm 0.234$ & DCD & & & & & & & \\
\hline 27072 & $2.804 \pm 0.276$ & $2.606 \pm 0.236$ & $2.508 \pm 0.228$ & DDD & 2.696 & 2.462 & 2.410 & 2.390 & 2.367 & 2.421 & $1,2,3,6,8$ \\
\hline 37279 & $-0.498 \pm 0.151$ & $-0.666 \pm 0.270$ & $-0.658 \pm 0.322$ & BDD & -0.420 & -0.576 & -0.664 & -0.670 & & -0.670 & $2,3,6,9$ \\
\hline 47080 & $4.059 \pm 0.410$ & $3.718 \pm 0.338$ & $3.690 \pm 0.018$ & DDE & 4.140 & 3.770 & 3.700 & & & & 2 \\
\hline 56997 & $3.988 \pm 0.242$ & $3.648 \pm 0.228$ & $3.588 \pm 0.036$ & DDE & & & & & & & \\
\hline 57443 & $3.931 \pm 0.276$ & $3.490 \pm 0.238$ & $3.489 \pm 0.278$ & DDD & 3.745 & 3.377 & 3.312 & 3.261 & 3.246 & 3.341 & $1,2,3,6$ \\
\hline 57757 & $2.597 \pm 0.252$ & $2.363 \pm 0.230$ & $2.269 \pm 0.254$ & DDD & 2.602 & 2.347 & 2.316 & 2.300 & & & $2,5,6$ \\
\hline 59199 & $3.525 \pm 0.286$ & $3.364 \pm 0.218$ & $3.307 \pm 0.240$ & DDD & 3.337 & 3.195 & 3.165 & & & & 2 \\
\hline 61317 & $3.213 \pm 0.218$ & $2.905 \pm 0.198$ & $2.848 \pm 0.310$ & DCD & 3.195 & 2.900 & 2.813 & 2.770 & & 2.800 & 2,6 \\
\hline 61941 & $2.041 \pm 0.272$ & $1.925 \pm 0.224$ & $1.873 \pm 0.232$ & DDD & 2.081 & 1.922 & 1.876 & & 1.880 & 1.917 & 2 \\
\hline 64394 & $3.232 \pm 0.234$ & $2.992 \pm 0.192$ & $2.923 \pm 0.274$ & DCD & 3.204 & 2.918 & 2.878 & 2.870 & 2.880 & 2.908 & $2,4,6,8,9$ \\
\hline 64924 & $3.334 \pm 0.200$ & $2.974 \pm 0.176$ & $2.956 \pm 0.236$ & CCD & & & & & & & \\
\hline 70497 & $3.179 \pm 0.244$ & $2.980 \pm 0.216$ & $2.739 \pm 0.332$ & DCD & 3.087 & 2.853 & 2.820 & 2.803 & & & $2,5,6$ \\
\hline 72659 & $2.660 \pm 0.448$ & $2.253 \pm 0.698$ & $1.971 \pm 0.600$ & DDD & 3.984 & 3.000 & 3.395 & 3.479 & 3.715 & & 2,7 \\
\hline 73695 & $3.416 \pm 0.282$ & $3.125 \pm 0.228$ & $3.011 \pm 0.268$ & DDD & & & 3.100 & & & & 2 \\
\hline 77257 & $3.430 \pm 0.220$ & $3.070 \pm 0.216$ & $2.989 \pm 0.230$ & DCD & 3.434 & 3.122 & 3.043 & 2.950 & & & 2,6 \\
\hline 78072 & $3.149 \pm 0.242$ & $2.875 \pm 0.224$ & $2.703 \pm 0.314$ & DDD & 2.913 & 2.643 & 2.633 & 2.623 & & 2.640 & $2,5,6$ \\
\hline 80686 & $4.110 \pm 0.270$ & $3.646 \pm 0.226$ & $3.661 \pm 0.284$ & DDD & & & & & & & \\
\hline 89937 & $2.588 \pm 0.260$ & $2.372 \pm 0.188$ & $2.216 \pm 0.252$ & DCD & 2.510 & 2.200 & 2.179 & 2.160 & & & 2,5 \\
\hline 93017 & $3.847 \pm 0.254$ & $3.611 \pm 0.252$ & $3.655 \pm 0.042$ & DDE & & & & & & & \\
\hline 99825 & $4.112 \pm 0.294$ & $3.582 \pm 0.266$ & $3.501 \pm 0.232$ & DDD & 4.160 & 3.740 & 3.670 & 3.600 & & & 2,5 \\
\hline 102485 & $3.363 \pm 0.258$ & $3.104 \pm 0.184$ & $3.094 \pm 0.262$ & DCD & & & & & & & \\
\hline 105858 & $3.340 \pm 0.248$ & $2.992 \pm 0.224$ & $2.969 \pm 0.252$ & DDD & 3.276 & 2.988 & 2.920 & 2.882 & 2.890 & 2.923 & 2,3 \\
\hline 109176 & $2.954 \pm 0.222$ & $2.729 \pm 0.184$ & $2.564 \pm 0.290$ & DCD & & & & & & & \\
\hline 113283 & $4.533 \pm 0.037$ & $3.804 \pm 0.210$ & $3.805 \pm 0.240$ & $\mathrm{ECD}$ & 4.480 & 3.940 & 3.830 & 3.790 & & & 2,5 \\
\hline 116771 & $3.299 \pm 0.286$ & $2.988 \pm 0.240$ & $2.946 \pm 0.288$ & DDD & & & 2.825 & & & & 2 \\
\hline \multicolumn{12}{|c|}{ FGK stars $(15 \mathrm{pc}<d \leq 20 \mathrm{pc})$} \\
\hline 5862 & $4.094 \pm 0.346$ & $3.719 \pm 0.268$ & $3.782 \pm 0.268$ & DDD & 3.980 & 3.712 & 3.666 & 3.635 & & & 2,5 \\
\hline 17651 & $3.609 \pm 0.302$ & $3.393 \pm 0.236$ & $3.353 \pm 0.270$ & DDD & & & & & & & \\
\hline 36366 & $3.221 \pm 0.232$ & $3.156 \pm 0.246$ & $2.978 \pm 0.316$ & DDD & 3.517 & & 3.329 & 3.325 & 3.270 & & $2,6,8$ \\
\hline 44248 & $3.209 \pm 0.268$ & $3.082 \pm 0.254$ & $3.003 \pm 0.358$ & DDD & & & 2.880 & & & & 2 \\
\hline 46509 & $3.907 \pm 0.252$ & $3.582 \pm 0.210$ & $3.560 \pm 0.242$ & DCD & 3.750 & 3.540 & 3.500 & 3.490 & & & 2,5 \\
\hline 48113 & $3.961 \pm 0.318$ & $3.725 \pm 0.262$ & $3.609 \pm 0.017$ & DDE & 4.040 & 3.710 & 3.670 & & & & 2,5 \\
\hline 61174 & $3.609 \pm 0.250$ & $3.372 \pm 0.240$ & $3.372 \pm 0.302$ & DDD & 3.690 & 3.570 & 3.540 & 3.510 & 3.550 & 3.590 & 2 \\
\hline 64241 & $3.399 \pm 0.244$ & $3.266 \pm 0.218$ & $3.108 \pm 0.246$ & DDD & & & 3.200 & & & & \\
\hline 67153 & $3.585 \pm 0.268$ & $3.347 \pm 0.220$ & $3.410 \pm 0.248$ & DDD & & & & & & & \\
\hline 71284 & $3.561 \pm 0.250$ & $3.462 \pm 0.246$ & $3.336 \pm 0.324$ & DDD & 3.705 & 3.514 & 3.490 & 3.487 & 3.480 & 3.465 & $2,5,6,7,8,9,10$ \\
\hline 75312 & $4.052 \pm 0.226$ & $3.701 \pm 0.236$ & $3.714 \pm 0.216$ & DDC & & & & & & & \\
\hline 77760 & $2.940 \pm 0.174$ & $2.744 \pm 0.164$ & $2.583 \pm 0.202$ & $\mathrm{CCC}$ & 3.490 & 3.152 & 3.119 & 3.094 & 3.070 & & $2,4,5,6,7$ \\
\hline 88745 & $3.459 \pm 0.198$ & $3.242 \pm 0.190$ & $3.107 \pm 0.230$ & $\mathrm{CCD}$ & 3.660 & 3.500 & 3.550 & & & & 2 \\
\hline 104858 & $3.736 \pm 0.260$ & $3.410 \pm 0.216$ & $3.384 \pm 0.252$ & DCD & & & & & & & \\
\hline 112447 & $3.358 \pm 0.254$ & $3.078 \pm 0.214$ & $2.961 \pm 0.286$ & DCD & 3.239 & & 2.926 & 2.923 & 2.860 & & $2,6,8$ \\
\hline
\end{tabular}

Notes. 2MASS $J H K_{\mathrm{s}}$ are from the 2MASS Point Source Catalogue (II/246 in Vizier). Zero points for calibration from magnitudes to fluxes are from Cohen et al. (2003). Nomenclature: HIP 7751A = HD 10360 = p Eri A, HIP 7751B = HD $10361=$ p Eri B.

References. References for the $J H K L L^{\prime} M$ photometry: (1) Carter (1990), SAAO infrared standards: http://www.saao.ac.za/fileadmin/ files/links/IRstd.txt; (2) Gezari et al. (2000), http://ircatalog.gsfc . nasa.gov/, also at Vizier, Catalogue no. II/225. (only magnitudes flagged "M"); (3) Koornneef (1983); (4) CVF standards http://www.iac.es/telescopes/pages/es/inicio/utilidades.php\#CVF; (5) Aumann \& Probst (1991); (6) UKIRT very bright standards http://www.jach.hawaii.edu/UKIRT/astronomy/calib/phot_cal/ bright_stds.html; (7) Selby et al. (1988); (8) Glass (1975) ; (9) Old, bright, UKIRT standards http://www. jach.hawaii . edu/UKIRT/ astronomy/calib/phot_cal/ukirt_stds.html; (10) Elias et al. (1982). 
A\&A 593, A51 (2016)

Table C.6. DUNES_DB stars with $d \leq 20$ pc: AKARI 9 and $18 \mu \mathrm{m}$ fluxes and WISE W1, W3, and W4 photometry.

\begin{tabular}{|c|c|c|c|c|c|}
\hline \multirow[t]{2}{*}{ HIP } & \multicolumn{2}{|c|}{ AKARI } & \multicolumn{3}{|c|}{ WISE } \\
\hline & $\begin{array}{l}9 \mu \mathrm{m} \\
(\mathrm{mJy})\end{array}$ & $\begin{array}{c}18 \mu \mathrm{m} \\
(\mathrm{mJy})\end{array}$ & $\begin{array}{c}3.35 \mu \mathrm{m}(\mathrm{W} 1) \\
(\mathrm{mag})\end{array}$ & $\begin{array}{c}11.56 \mu \mathrm{m}(\mathrm{W} 3) \\
(\mathrm{mag})\end{array}$ & $\begin{array}{c}22.09 \mu \mathrm{m}(\mathrm{W} 4) \\
(\mathrm{mag})\end{array}$ \\
\hline \multicolumn{6}{|c|}{ FGK stars $(d \leq 15 \mathrm{pc})$} \\
\hline 1599 & $4193 \pm 28$ & $1055 \pm 30$ & & $2.856 \pm 0.011$ & $2.788 \pm 0.019$ \\
\hline 3765 & $2199 \pm 15$ & $519 \pm 8$ & & $3.370 \pm 0.039$ & $3.493 \pm 0.023$ \\
\hline 7751 & $3431 \pm 198$ & $892 \pm 20$ & & & $3.095 \pm 0.053$ \\
\hline 7751B & $1810 \pm 135$ & & & & \\
\hline 7918 & $2280 \pm 24$ & $494 \pm 12$ & $3.489 \pm 0.423$ & $3.469 \pm 0.012$ & $3.444 \pm 0.018$ \\
\hline 7981 & $2724 \pm 5$ & $586 \pm 39$ & $3.274 \pm 0.479$ & & $3.312 \pm 0.033$ \\
\hline 10644 & $2890 \pm 17$ & $668 \pm 39$ & & $3.187 \pm 0.012$ & $3.221 \pm 0.024$ \\
\hline 12114 & $2506 \pm 19$ & $486 \pm 20$ & & $3.273 \pm 0.020$ & $3.383 \pm 0.019$ \\
\hline 12843 & $2618 \pm 9$ & $623 \pm 83$ & & $3.336 \pm 0.010$ & $3.243 \pm 0.019$ \\
\hline 14879 & $5683 \pm 13$ & $1346 \pm 11$ & $2.653 \pm 0.163$ & $2.360 \pm 0.004$ & $2.440 \pm 0.015$ \\
\hline 15457 & $2789 \pm 21$ & $619 \pm 45$ & $3.263 \pm 0.461$ & $3.277 \pm 0.010$ & $3.240 \pm 0.020$ \\
\hline 15510 & $5692 \pm 25$ & $1271 \pm 21$ & & & \\
\hline 16852 & $3903 \pm 5$ & $850 \pm 21$ & & $2.905 \pm 0.015$ & $2.868 \pm 0.021$ \\
\hline 22449 & $8357 \pm 36$ & $1876 \pm 61$ & & $2.136 \pm 0.012$ & $2.090 \pm 0.018$ \\
\hline 23693 & $2402 \pm 6$ & $583 \pm 14$ & $3.425 \pm 0.488$ & $3.424 \pm 0.011$ & $3.336 \pm 0.019$ \\
\hline 27072 & $6176 \pm 34$ & & $3.085 \pm 0.017$ & $2.261 \pm 0.006$ & $2.394 \pm 0.014$ \\
\hline 37279 & $109400 \pm 1730$ & $22930 \pm 197$ & & $-0.425 \pm 0.427$ & $-0.637 \pm 0.005$ \\
\hline 47080 & $2004 \pm 26$ & $483 \pm 14$ & & $3.642 \pm 0.013$ & $3.608 \pm 0.021$ \\
\hline 56997 & $2044 \pm 15$ & $434 \pm 31$ & $3.572 \pm 0.397$ & $3.743 \pm 0.053$ & $3.584 \pm 0.031$ \\
\hline 57443 & $2857 \pm 14$ & $624 \pm 9$ & & $3.302 \pm 0.013$ & $3.241 \pm 0.025$ \\
\hline 57757 & $6914 \pm 6$ & $1558 \pm 23$ & & $2.386 \pm 0.013$ & $2.294 \pm 0.016$ \\
\hline 59199 & $3027 \pm 10$ & $707 \pm 35$ & & $3.189 \pm 0.013$ & $3.113 \pm 0.017$ \\
\hline 61317 & $4325 \pm 20$ & $993 \pm 23$ & & $2.733 \pm 0.026$ & $2.764 \pm 0.020$ \\
\hline 61941 & $10480 \pm 138$ & $2225 \pm 43$ & & $1.971 \pm 0.012$ & $1.858 \pm 0.015$ \\
\hline 64394 & $3975 \pm 30$ & $900 \pm 20$ & & $2.858 \pm 0.013$ & $2.827 \pm 0.017$ \\
\hline 64924 & $3380 \pm 34$ & $838 \pm 42$ & & $3.091 \pm 0.009$ & $2.986 \pm 0.019$ \\
\hline 70497 & $4156 \pm 19$ & $993 \pm 19$ & $3.149 \pm 0.058$ & $2.606 \pm 0.004$ & $2.777 \pm 0.013$ \\
\hline 72659 & $5454 \pm 26$ & $1233 \pm 53$ & $2.788 \pm 0.310$ & $2.887 \pm 0.026$ & $2.831 \pm 0.024$ \\
\hline 73695 & $3370 \pm 41$ & $790 \pm 35$ & & $3.060 \pm 0.009$ & $3.049 \pm 0.017$ \\
\hline 77257 & $3590 \pm 37$ & $776 \pm 10$ & & $3.000 \pm 0.011$ & $2.971 \pm 0.020$ \\
\hline 78072 & $4911 \pm 18$ & $1064 \pm 34$ & & $2.677 \pm 0.007$ & $2.628 \pm 0.018$ \\
\hline 80686 & $2155 \pm 18$ & $513 \pm 25$ & $3.582 \pm 0.317$ & $3.536 \pm 0.013$ & $3.511 \pm 0.021$ \\
\hline 89937 & $7653 \pm 17$ & $1701 \pm 15$ & & $2.252 \pm 0.014$ & $2.154 \pm 0.016$ \\
\hline 93017 & $1896 \pm 6$ & $432 \pm 35$ & $3.708 \pm 0.386$ & $3.679 \pm 0.014$ & $3.641 \pm 0.019$ \\
\hline 99825 & & $448 \pm 5$ & $3.684 \pm 0.505$ & $3.587 \pm 0.024$ & $3.645 \pm 0.022$ \\
\hline 102485 & $3252 \pm 17$ & $775 \pm 26$ & & $3.145 \pm 0.014$ & $3.104 \pm 0.021$ \\
\hline 105858 & $3845 \pm 27$ & $912 \pm 15$ & $3.706 \pm 0.244$ & $4.239 \pm 0.013$ & $2.761 \pm 0.016$ \\
\hline 109176 & $5028 \pm 18$ & $1184 \pm 40$ & & $2.671 \pm 0.007$ & $2.619 \pm 0.020$ \\
\hline 113283 & $1716 \pm 9$ & & $3.897 \pm 0.411$ & $3.698 \pm 0.010$ & $3.770 \pm 0.021$ \\
\hline 116771 & $4124 \pm 59$ & $863 \pm 15$ & & $2.911 \pm 0.013$ & $2.859 \pm 0.021$ \\
\hline \multicolumn{6}{|c|}{ FGK stars $(15 \mathrm{pc}<d \leq 20 \mathrm{pc})$} \\
\hline 5862 & $1940 \pm 9$ & $377 \pm 4$ & & $3.652 \pm 0.013$ & $3.613 \pm 0.025$ \\
\hline 17651 & $3003 \pm 37$ & $719 \pm 34$ & $3.209 \pm 0.530$ & $3.173 \pm 0.009$ & $3.134 \pm 0.022$ \\
\hline 36366 & $2767 \pm 30$ & $559 \pm 23$ & $3.353 \pm 0.389$ & $3.322 \pm 0.009$ & $3.233 \pm 0.020$ \\
\hline 44248 & $3965 \pm 43$ & $904 \pm 43$ & & $2.904 \pm 0.015$ & $2.839 \pm 0.018$ \\
\hline 46509 & & $550 \pm 25$ & & $3.521 \pm 0.012$ & $3.457 \pm 0.020$ \\
\hline 48113 & $1930 \pm 14$ & $458 \pm 36$ & $3.621 \pm 0.377$ & $3.656 \pm 0.015$ & $3.619 \pm 0.022$ \\
\hline 61174 & $2631 \pm 15$ & $837 \pm 6$ & & $3.308 \pm 0.016$ & $2.752 \pm 0.023$ \\
\hline 64241 & $2976 \pm 9$ & $696 \pm 25$ & & $3.219 \pm 0.012$ & $3.165 \pm 0.024$ \\
\hline 67153 & $2645 \pm 13$ & $657 \pm 30$ & & $3.344 \pm 0.012$ & $3.272 \pm 0.019$ \\
\hline 71284 & $2226 \pm 19$ & $519 \pm 31$ & $3.478 \pm 0.415$ & $3.507 \pm 0.010$ & $3.444 \pm 0.019$ \\
\hline 75312 & $1960 \pm 9$ & $465 \pm 24$ & $3.649 \pm 0.503$ & $3.651 \pm 0.015$ & $3.608 \pm 0.021$ \\
\hline 77760 & $3202 \pm 10$ & $717 \pm 21$ & & $3.115 \pm 0.010$ & $3.073 \pm 0.019$ \\
\hline 88745 & $2085 \pm 17$ & $520 \pm 22$ & $3.587 \pm 0.358$ & $3.578 \pm 0.015$ & $3.552 \pm 0.022$ \\
\hline 104858 & $2791 \pm 32$ & $658 \pm 7$ & & $3.290 \pm 0.013$ & $3.247 \pm 0.026$ \\
\hline 112447 & $3970 \pm 36$ & $925 \pm 32$ & & $2.910 \pm 0.013$ & $2.884 \pm 0.021$ \\
\hline
\end{tabular}

Notes. AKARI 9 and $18 \mu \mathrm{m}$ fluxes and uncertainties are from the AKARI/IRC mid-IR all-sky Survey (ISAS/ JAXA, 2010, catalogue II/297 in Vizier). WISE data are from the AllWISE Data Release (Cutri et al. 2013), Vizier catalogue II/328, except for HIP 14879, HIP 27072, HIP 70497, and HIP 72659, which are from the WISE All-Sky Data Release (Cutri et al. 2012, Vizier catalogue II/311/wise). Zero points for calibration are from Wright et al. (2010). Nomenclature: HIP 7751 = HD 10360+HD $10361=$ p Eri A+p Eri B, HIP 7751B = HD $10361=$ p Eri B. 
B. Montesinos et al.: Debris discs around nearby FGK main-sequence stars

Table C.7. DUNES_DB stars with $d \leq 20$ pc: IRAS 12, 25, $60 \mu \mathrm{m}$ and Spitzer/MIPS 24 and $70 \mu \mathrm{m}$ fluxes.

\begin{tabular}{|c|c|c|c|c|c|c|c|}
\hline \multirow[t]{2}{*}{ HIP } & \multicolumn{5}{|c|}{ IRAS } & \multicolumn{2}{|c|}{ MIPS } \\
\hline & $\begin{array}{c}12 \mu \mathrm{m} \\
(\mathrm{mJy})\end{array}$ & $\%$ & $\begin{array}{c}25 \mu \mathrm{m} \\
(\mathrm{mJy})\end{array}$ & $\%$ & $\begin{array}{c}60 \mu \mathrm{m} \\
(\mathrm{mJy})\end{array}$ & $\begin{array}{l}24 \mu \mathrm{m} \\
(\mathrm{mJy})\end{array}$ & $\begin{array}{l}70 \mu \mathrm{m} \\
(\mathrm{mJy})\end{array}$ \\
\hline \multicolumn{8}{|c|}{ FGK stars $(d \leq 15 \mathrm{pc})$} \\
\hline 1599 & $3.11 \times 10^{3}$ & 4 & $6.95 \times 10^{2}$ & 4 & & $519 \pm 11$ & $82 \pm 8$ \\
\hline 3765 & $1.56 \times 10^{3}$ & 7 & & & & $264 \pm 5$ & $26 \pm 10$ \\
\hline 7751 & $2.70 \times 10^{3}$ & 5 & $6.55 \times 10^{2}$ & 7 & & $231 \pm 5$ & $22 \pm 4$ \\
\hline 7918 & $1.67 \times 10^{3}$ & 5 & $3.87 \times 10^{2}$ & 8 & & $289 \pm 6$ & $25 \pm 11$ \\
\hline 7981 & $1.87 \times 10^{3}$ & 7 & $6.38 \times 10^{2} 1$ & & & $334 \pm 7$ & $48 \pm 9$ \\
\hline 10644 & $2.11 \times 10^{3}$ & 5 & $4.97 \times 10^{2}$ & 6 & & $359 \pm 7$ & $43 \pm 5$ \\
\hline 12114 & $1.83 \times 10^{3}$ & 5 & $3.47 \times 10^{2} 1$ & & & $311 \pm 6$ & $35 \pm 6$ \\
\hline 12843 & $1.93 \times 10^{3}$ & 6 & & & & $346 \pm 7$ & $37 \pm 13$ \\
\hline 14879 & $4.02 \times 10^{3}$ & 4 & $9.43 \times 10^{2}$ & 4 & $1.80 \times 10^{2} 18$ & $754 \pm 15$ & $105 \pm 13$ \\
\hline 15457 & $2.05 \times 10^{3}$ & 4 & $4.67 \times 10^{2}$ & 8 & & $340 \pm 7$ & $39 \pm 8$ \\
\hline 15510 & $4.27 \times 10^{3}$ & 4 & $9.50 \times 10^{2}$ & 5 & $1.89 \times 10^{2} 17$ & $722 \pm 15$ & $100 \pm 8$ \\
\hline 16852 & $2.85 \times 10^{3}$ & 5 & $6.81 \times 10^{2}$ & 8 & & $502 \pm 10$ & $106 \pm 9$ \\
\hline 22449 & $5.67 \times 10^{3} 1$ & & $1.51 \times 10^{3}$ & 7 & $2.04 \times 10^{2} 20$ & $1020 \pm 21$ & $116 \pm 9$ \\
\hline 23693 & $1.67 \times 10^{3}$ & 5 & $4.40 \times 10^{2}$ & 8 & & $308 \pm 6$ & $6 \pm 7$ \\
\hline 27072 & $4.40 \times 10^{3}$ & 5 & $9.81 \times 10^{2}$ & 6 & $2.28 \times 10^{2} 16$ & $765 \pm 16$ & $56 \pm 10$ \\
\hline 37279 & $8.29 \times 10^{4}$ & 4 & $1.87 \times 10^{4}$ & 5 & $3.00 \times 10^{3} \quad 6$ & & $1520 \pm 103$ \\
\hline 47080 & $1.47 \times 10^{3}$ & 5 & $3.49 \times 10^{2} 1$ & & & $257 \pm 5$ & $581 \pm 0$ \\
\hline 56997 & & & & & & $258 \pm 5$ & $30 \pm 5$ \\
\hline 57443 & $2.03 \times 10^{3}$ & 7 & $4.92 \times 10^{2} 1$ & & & $334 \pm 7$ & $32 \pm 6$ \\
\hline 57757 & $5.24 \times 10^{3}$ & 7 & $1.21 \times 10^{3} 1$ & & $1.79 \times 10^{2} 25$ & $829 \pm 17$ & $114 \pm 1$ \\
\hline 59199 & $2.45 \times 10^{3}$ & 7 & $5.52 \times 10^{2}$ & 9 & & $368 \pm 8$ & $46 \pm 6$ \\
\hline 61317 & $3.49 \times 10^{3}$ & 5 & $7.29 \times 10^{2}$ & 6 & & $557 \pm 11$ & $65 \pm 1$ \\
\hline 61941 & $7.67 \times 10^{3}$ & 6 & $1.70 \times 10^{3}$ & 7 & $2.91 \times 10^{2} 20$ & $1410 \pm 29$ & $238 \pm 122$ \\
\hline 64394 & $2.98 \times 10^{3}$ & 7 & $6.46 \times 10^{2}$ & 8 & & $489 \pm 10$ & $49 \pm 6$ \\
\hline 64924 & $2.56 \times 10^{3}$ & 6 & $5.80 \times 10^{2} 1$ & & & $443 \pm 9$ & $192 \pm 16$ \\
\hline 70497 & $3.08 \times 10^{3}$ & 4 & $7.24 \times 10^{2}$ & 5 & $1.09 \times 10^{2} 28$ & $540 \pm 11$ & $63 \pm 8$ \\
\hline 72659 & $3.90 \times 10^{3}$ & 5 & $8.95 \times 10^{2}$ & 6 & $1.57 \times 10^{2} 25$ & $476 \pm 10$ & $53 \pm 7$ \\
\hline 73695 & $2.50 \times 10^{3}$ & 4 & $5.88 \times 10^{2}$ & 5 & & $468 \pm 10$ & $70 \pm 12$ \\
\hline 77257 & $2.46 \times 10^{3}$ & 4 & $5.91 \times 10^{2}$ & 7 & & $433 \pm 9$ & $84 \pm 15$ \\
\hline 78072 & $3.82 \times 10^{3}$ & 5 & $8.82 \times 10^{2}$ & 5 & $3.32 \times 10^{2} 12$ & $630 \pm 13$ & $70 \pm 17$ \\
\hline 80686 & $1.58 \times 10^{3}$ & 4 & $3.68 \times 10^{2}$ & 6 & & $284 \pm 6$ & $21 \pm 13$ \\
\hline 89937 & $5.51 \times 10^{3}$ & 3 & $1.31 \times 10^{3}$ & 4 & $2.20 \times 10^{2} 14$ & $1050 \pm 21$ & $127 \pm 10$ \\
\hline 93017 & $1.44 \times 10^{3}$ & 4 & $3.47 \times 10^{2}$ & 6 & & $239 \pm 5$ & $25 \pm 6$ \\
\hline 99825 & $1.49 \times 10^{3}$ & 6 & $4.04 \times 10^{2} 2$ & & & $237 \pm 5$ & $22 \pm 4$ \\
\hline 102485 & $2.35 \times 10^{3}$ & 7 & $5.14 \times 10^{2} 1$ & & & $391 \pm 8$ & $44 \pm 8$ \\
\hline 105858 & $2.95 \times 10^{3}$ & 4 & $6.42 \times 10^{2}$ & 6 & $1.72 \times 10^{2} 21$ & $472 \pm 10$ & $44 \pm 6$ \\
\hline 109176 & $3.70 \times 10^{3}$ & 7 & $8.55 \times 10^{2} 1$ & & & $667 \pm 14$ & $75 \pm 7$ \\
\hline 113283 & $1.27 \times 10^{3}$ & 6 & $3.45 \times 10^{2} 1$ & & & $213 \pm 4$ & $27 \pm 4$ \\
\hline 116771 & $2.97 \times 10^{3}$ & 6 & $7.37 \times 10^{2} 1$ & 15 & & $511 \pm 10$ & $61 \pm 8$ \\
\hline \multicolumn{8}{|c|}{ FGK stars $(15 \mathrm{pc}<d \leq 20 \mathrm{pc})$} \\
\hline 5862 & $1.44 \times 10^{3}$ & 7 & $3.45 \times 10^{2} 1$ & & & $246 \pm 5$ & $45 \pm 5$ \\
\hline 17651 & $2.19 \times 10^{3}$ & 5 & $4.76 \times 10^{2}$ & 7 & & $366 \pm 8$ & $48 \pm 5$ \\
\hline 36366 & $2.01 \times 10^{3}$ & 5 & $4.82 \times 10^{2} 2$ & & & $356 \pm 7$ & $42 \pm 7$ \\
\hline 44248 & $2.94 \times 10^{3}$ & 5 & $7.41 \times 10^{2}$ & 7 & & $518 \pm 11$ & \\
\hline 46509 & $1.69 \times 10^{3}$ & 5 & $3.37 \times 10^{2} 1$ & 14 & & $258 \pm 5$ & $31 \pm 4$ \\
\hline 48113 & $1.48 \times 10^{3}$ & 5 & $3.27 \times 10^{2}$ & 9 & & $247 \pm 5$ & $33 \pm 5$ \\
\hline 61174 & $2.24 \times 10^{3}$ & 6 & $7.70 \times 10^{2}$ & 8 & $3.08 \times 10^{2} 14$ & $573 \pm 12$ & $20 \pm 14$ \\
\hline 64241 & $2.19 \times 10^{3}$ & 6 & $5.11 \times 10^{2} 1$ & 13 & & $385 \pm 8$ & $50 \pm 11$ \\
\hline 67153 & $1.93 \times 10^{3}$ & 5 & $4.22 \times 10^{2} 1$ & 10 & & $332 \pm 7$ & $106 \pm 76$ \\
\hline 71284 & $1.61 \times 10^{3}$ & 5 & $4.13 \times 10^{2}$ & 7 & $1.40 \times 10^{2} 25$ & $302 \pm 6$ & $72 \pm 11$ \\
\hline 75312 & $1.43 \times 10^{3}$ & 5 & $3.41 \times 10^{2}$ & 9 & & $273 \pm 6$ & $70 \pm 12$ \\
\hline 77760 & $2.31 \times 10^{3}$ & 5 & $5.71 \times 10^{2}$ & 6 & & $402 \pm 8$ & $40 \pm 6$ \\
\hline 88745 & $1.52 \times 10^{3}$ & 5 & $3.50 \times 10^{2}$ & 8 & & $268 \pm 6$ & $107 \pm 9$ \\
\hline 104858 & $2.21 \times 10^{3}$ & 7 & $5.42 \times 10^{2}$ & 8 & & $362 \pm 7$ & $43 \pm 5$ \\
\hline 112447 & $3.05 \times 10^{3}$ & 7 & $7.56 \times 10^{2}$ & 9 & & $467 \pm 10$ & $50 \pm 5$ \\
\hline
\end{tabular}

Notes. All IRAS fluxes are from the Faint Source Catalogue (II/156A in Vizier) except for HIP 3765, which is from the IRAS Catalogue of Point Sources (II/125). Upper limits to the fluxes are not given. Spitzer/MIPS fluxes at 24 and $70 \mu \mathrm{m}$ were extracted by members of the DUNES team. See http://sdc.cab.inta-csic.es/dunes/HELP/DUNES_Archive_ReadMe.jsp or http://sdc.cab.inta-csic.es/dunes/HELP/DUNES_ Archive_ReadMe.pdf for details. 
A\&A 593, A51 (2016)

Table C.8. OBSIDs for the DUNES_DB stars with $d \leq 20 \mathrm{pc}$.

\begin{tabular}{|c|c|c|c|c|c|}
\hline HIP & $\mathrm{HD}$ & PACS & Scan & X-Scan & On-source time [s] \\
\hline \multicolumn{6}{|c|}{ FGK stars $(d<15 \mathrm{pc})$} \\
\hline 1599 & 1581 & $100 / 160$ & 1342198529 & 1342198530 & 288 \\
\hline 3765 & 4628 & $100 / 160$ & 1342213215 & 1342213216 & 288 \\
\hline 7751 & 10360 & $100 / 160$ & 1342193169 & 1342193170 & 288 \\
\hline 7751 & 10361 & $100 / 160$ & 1342193169 & 1342193170 & 288 \\
\hline 7918 & 10307 & $100 / 160$ & 1342223568 & 1342223569 & 288 \\
\hline 7981 & 10476 & $100 / 160$ & 1342213522 & 1342213523 & 288 \\
\hline 10644 & 13974 & $100 / 160$ & 1342223878 & 1342223879 & 288 \\
\hline 12114 & 16160 & $100 / 160$ & 1342215727 & 1342215728 & 288 \\
\hline 12843 & 17206 & $100 / 160$ & 1342214006 & 1342214007 & 288 \\
\hline 14879 & 20010 & $100 / 160$ & 1342202298 & 1342202299 & 288 \\
\hline 15457 & 20630 & $100 / 160$ & 1342216133 & 1342216134 & 288 \\
\hline 15510 & 20794 & $100 / 160$ & 1342216456 & 1342216457 & 1080 \\
\hline 16852 & 22484 & $100 / 160$ & 1342224212 & 1342224213 & 288 \\
\hline 22449 & 30652 & $100 / 160$ & 1342193142 & 1342193143 & 288 \\
\hline 23693 & 33262 & $100 / 160$ & 1342205208 & 1342205209 & 288 \\
\hline 27072 & 38393 & $100 / 160$ & 1342203715 & 1342203716 & 288 \\
\hline 37279 & 61421 & $100 / 160$ & 1342208989 & 1342208990 & 1080 \\
\hline 47080 & 82885 & $100 / 160$ & 1342209372 & 1342209373 & 288 \\
\hline 56997 & 101501 & $100 / 160$ & 1342211430 & 1342211431 & 288 \\
\hline 57443 & 102365 & $100 / 160$ & 1342202240 & 1342202241 & 288 \\
\hline 57757 & 102870 & $100 / 160$ & 1342212383 & 1342212384 & 288 \\
\hline 59199 & 105452 & $100 / 160$ & 1342212824 & 1342212825 & 288 \\
\hline 61317 & 109358 & $100 / 160$ & 1342208821 & 1342208822 & 288 \\
\hline 61941 & $110379 \mathrm{~J}$ & $100 / 160$ & 1342212644 & 1342212645 & 288 \\
\hline 64394 & 114710 & $100 / 160$ & 1342212672 & 1342212672 & 288 \\
\hline 64924 & 115617 & $100 / 160$ & 1342202551 & 1342202552 & 288 \\
\hline 70497 & 126660 & $100 / 160$ & 1342209638 & 1342209639 & 288 \\
\hline 72659 & 131156 & $100 / 160$ & 1342213800 & 1342213801 & 288 \\
\hline 73695 & 133640 & $100 / 160$ & 1342208966 & 1342208967 & 288 \\
\hline 77257 & 141004 & $100 / 160$ & 1342204158 & 1342204159 & 288 \\
\hline 78072 & 142860 & $100 / 160$ & 1342215378 & 1342215379 & 288 \\
\hline 80686 & 147584 & $100 / 160$ & 1342216577 & 1342216578 & 288 \\
\hline 89937 & 170153 & $100 / 160$ & 1342220810 & 1342220811 & 288 \\
\hline 93017 & 176051 & $100 / 160$ & 1342205038 & 1342205039 & 288 \\
\hline 99825 & 192310 & $100 / 160$ & 1342208466 & 1342208467 & 288 \\
\hline 102485 & 197692 & $100 / 160$ & 1342193530 & 1342193531 & 288 \\
\hline 105858 & 203608 & $100 / 160$ & 1342215352 & 1342215353 & 288 \\
\hline 109176 & 210027 & $100 / 160$ & 1342198503 & 1342198504 & 288 \\
\hline 113283 & 216803 & $100 / 160$ & 1342211140 & 1342211141 & 288 \\
\hline 116771 & 222368 & $100 / 160$ & 1342198507 & 1342198508 & 288 \\
\hline \multicolumn{6}{|c|}{ FGK stars $(15 \mathrm{pc}<d \leq 20 \mathrm{pc})$} \\
\hline 5862 & 7570 & $100 / 160$ & 1342213175 & 1342213176 & 288 \\
\hline 17651 & 23754 & $100 / 160$ & 1342223596 & 1342223596 & 288 \\
\hline 36366 & 58946 & $100 / 160$ & 1342219410 & 1342219411 & 288 \\
\hline 44248 & 76943 & $100 / 160$ & 1342208506 & 1342208507 & 288 \\
\hline 46509 & 81997 & $100 / 160$ & 1342209475 & 1342209476 & 288 \\
\hline 48113 & 84737 & $100 / 160$ & 1342210446 & 1342210447 & 288 \\
\hline 61174 & 109085 & $100 / 160$ & 1342234385 & 1342234386 & 288 \\
\hline 64241 & $114378 \mathrm{~J}$ & $100 / 160$ & 1342212720 & 1342212721 & 288 \\
\hline 67153 & 119756 & $100 / 160$ & 1342203109 & 1342203110 & 288 \\
\hline 71284 & 128167 & $100 / 160$ & 1342213660 & 1342213661 & 288 \\
\hline 75312 & $137107 \mathrm{~J}$ & $100 / 160$ & 1342213788 & 1342213789 & 288 \\
\hline 77760 & 142373 & $100 / 160$ & 1342205167 & 1342205168 & 288 \\
\hline 88745 & 165908 & $100 / 160$ & 1342195362 & 1342195363 & 288 \\
\hline 104858 & 202275 & $100 / 160$ & 1342195606 & 1342195607 & 288 \\
\hline 112447 & 215648 & $100 / 160$ & 1342198505 & 1342198506 & 288 \\
\hline
\end{tabular}


B. Montesinos et al.: Debris discs around nearby FGK main-sequence stars

Table C.9. PACS flux densities for the DUNES_DB stars with $d \leq 15 \mathrm{pc}$ and $15 \mathrm{pc}<d \leq 20 \mathrm{pc}$.

\begin{tabular}{|c|c|c|c|c|c|c|c|c|}
\hline HIP & HD & $\begin{array}{c}\text { PACS100 } \\
\text { (mJy) }\end{array}$ & $\begin{array}{l}\text { S100 } \\
(\mathrm{mJy})\end{array}$ & $\chi_{100}$ & $\begin{array}{c}\text { PACS160 } \\
(\mathrm{mJy})\end{array}$ & $\begin{array}{l}\text { S160 } \\
(\mathrm{mJy})\end{array}$ & $\chi_{160}$ & Status \\
\hline \multicolumn{9}{|c|}{ FGK stars $(d \leq 15 \mathrm{pc})$} \\
\hline 1599 & 1581 & $33.23 \pm 2.35$ & $30.08 \pm 0.19$ & 1.34 & $29.03 \pm 3.46$ & $11.75 \pm 0.07$ & 4.99 & Dubious \\
\hline 3765 & 4628 & $19.43 \pm 1.93$ & $15.84 \pm 0.36$ & 1.83 & $<3.38$ & $6.19 \pm 0.14$ & & No excess \\
\hline & 10360 & $11.95 \pm 1.70$ & $12.39 \pm 0.21$ & -0.26 & $<5.00$ & $4.84 \pm 0.08$ & & No excess \\
\hline 7751 & 10361 & $10.66 \pm 1.68$ & $14.90 \pm 0.26$ & -2.49 & $<5.00$ & $5.82 \pm 0.10$ & & No excess \\
\hline 7918 & 10307 & $14.33 \pm 1.75$ & $16.21 \pm 0.25$ & -1.06 & $10.35 \pm 3.17$ & $6.33 \pm 0.10$ & 1.27 & No excess \\
\hline 7981 & 10476 & $20.18 \pm 1.91$ & $20.05 \pm 0.21$ & 0.07 & $7.87 \pm 2.05$ & $7.83 \pm 0.08$ & 0.02 & No excess \\
\hline 10644 & 13974 & $23.45 \pm 2.07$ & $20.75 \pm 0.30$ & 1.29 & $6.59 \pm 3.60$ & $8.11 \pm 0.12$ & -0.42 & No excess \\
\hline 12114 & 16160 & $15.29 \pm 1.74$ & $17.26 \pm 0.24$ & -1.12 & $5.91 \pm 3.32$ & $6.74 \pm 0.09$ & -0.25 & No excess \\
\hline 12843 & 17206 & $20.94 \pm 2.08$ & $19.10 \pm 0.22$ & 0.88 & $7.52 \pm 3.18$ & $7.46 \pm 0.09$ & 0.02 & No excess \\
\hline 14879 & 20010 & $40.72 \pm 2.70$ & $38.77 \pm 1.48$ & 0.63 & $24.90 \pm 3.58$ & $15.14 \pm 0.58$ & 2.69 & No excess \\
\hline 15457 & 20630 & $24.78 \pm 2.02$ & $19.85 \pm 0.24$ & 2.43 & $12.14 \pm 2.52$ & $7.75 \pm 0.09$ & 1.74 & No excess \\
\hline $15510^{*}$ & 20794 & $48.62 \pm 2.64$ & $40.60 \pm 0.34$ & 3.01 & $27.12 \pm 2.33$ & $15.86 \pm 0.13$ & 4.82 & Excess \\
\hline $16852^{*}$ & 22484 & $67.30 \pm 3.64$ & $28.09 \pm 0.34$ & 10.71 & $33.35 \pm 3.19$ & $10.97 \pm 0.13$ & 7.01 & Excess \\
\hline 22449 & 30652 & $65.99 \pm 3.67$ & $58.79 \pm 0.29$ & 1.95 & & $22.97 \pm 0.12$ & & No excess \\
\hline 23693 & 33262 & $33.97 \pm 2.33$ & $17.49 \pm 0.42$ & 6.97 & $9.39 \pm 2.84$ & $6.83 \pm 0.17$ & 0.90 & Excess \\
\hline 27072 & 38393 & $49.21 \pm 2.95$ & $43.99 \pm 0.29$ & 1.76 & $19.67 \pm 3.13$ & $17.18 \pm 0.11$ & 0.80 & No excess \\
\hline 37279 & 61421 & $867.50 \pm 43.38$ & $733.50 \pm 8.48$ & 3.03 & $355.75 \pm 17.88$ & $286.50 \pm 3.31$ & 3.81 & Dubious \\
\hline 47080 & 82885 & $10.12 \pm 1.87$ & $13.56 \pm 0.21$ & -1.83 & $8.30 \pm 3.16$ & $5.30 \pm 0.08$ & 0.94 & No excess \\
\hline 56997 & 101501 & $15.43 \pm 1.71$ & $14.83 \pm 0.14$ & 0.35 & $12.69 \pm 3.51$ & $5.79 \pm 0.05$ & 1.97 & No excess \\
\hline 57443 & 102365 & $16.22 \pm 1.96$ & $19.83 \pm 0.17$ & -1.84 & $<3.85$ & $7.74 \pm 0.07$ & & No excess \\
\hline 57757 & 102870 & $68.43 \pm 3.80$ & $48.38 \pm 0.23$ & 5.26 & $40.67 \pm 3.83$ & $18.90 \pm 0.09$ & 5.68 & Excess \\
\hline 59199 & 105452 & $27.10 \pm 2.16$ & $21.65 \pm 0.34$ & 2.49 & $25.40 \pm 3.29$ & $8.46 \pm 0.13$ & 5.14 & Dubious \\
\hline 61317 & 109358 & $31.43 \pm 2.34$ & $30.76 \pm 0.30$ & 0.28 & $9.52 \pm 3.46$ & $12.02 \pm 0.12$ & -0.72 & No excess \\
\hline 61941 & $110379 \mathrm{~J}$ & $85.77 \pm 4.59$ & $71.92 \pm 0.86$ & 2.97 & $49.43 \pm 3.86$ & $28.10 \pm 0.34$ & 5.50 & Dubious \\
\hline 64394 & 114710 & $23.74 \pm 1.98$ & $28.55 \pm 0.25$ & -2.41 & $13.50 \pm 3.26$ & $11.15 \pm 0.10$ & 0.72 & No excess \\
\hline $64924^{*}$ & 115617 & $211.56 \pm 10.74$ & $23.67 \pm 1.10$ & 17.40 & $161.27 \pm 8.74$ & $9.24 \pm 0.43$ & 17.37 & Excess \\
\hline 70497 & 126660 & $36.36 \pm 2.46$ & $30.16 \pm 0.33$ & 2.50 & $15.57 \pm 3.17$ & $11.78 \pm 0.13$ & 1.20 & No excess \\
\hline 72659 & 131156 & $57.51 \pm 7.46$ & $27.42 \pm 0.55$ & 4.02 & $42.14 \pm 8.58$ & $10.71 \pm 0.21$ & 3.66 & Dubious \\
\hline 73695 & 133640 & $32.17 \pm 2.15$ & $24.01 \pm 0.25$ & 3.77 & $30.83 \pm 2.94$ & $9.38 \pm 0.10$ & 7.30 & Dubious \\
\hline 77257 & 141004 & $33.11 \pm 2.35$ & $25.19 \pm 0.42$ & 3.32 & $20.23 \pm 3.12$ & $9.84 \pm 0.16$ & 3.33 & Dubious \\
\hline 78072 & 142860 & $30.71 \pm 2.26$ & $35.81 \pm 0.20$ & -2.25 & $24.40 \pm 3.91$ & $13.99 \pm 0.08$ & 2.66 & No excess \\
\hline 80686 & 147584 & $17.31 \pm 1.75$ & $15.45 \pm 0.28$ & 1.05 & $<3.92$ & $6.03 \pm 0.11$ & & No excess \\
\hline 89937 & 170153 & $57.97 \pm 3.27$ & $55.98 \pm 0.31$ & 0.61 & $32.41 \pm 3.72$ & $21.87 \pm 0.12$ & 2.83 & No excess \\
\hline 93017 & 176051 & $18.73 \pm 1.79$ & $13.38 \pm 0.26$ & 2.95 & $<5.02$ & $5.23 \pm 0.10$ & & No excess \\
\hline 99825 & 192310 & $11.79 \pm 1.87$ & $14.17 \pm 0.16$ & -1.27 & $8.32 \pm 3.35$ & $5.53 \pm 0.06$ & 0.83 & No excess \\
\hline 102485 & 197692 & $27.11 \pm 2.35$ & $23.05 \pm 0.20$ & 1.72 & $9.13 \pm 3.09$ & $9.00 \pm 0.08$ & 0.04 & No excess \\
\hline 105858 & 203608 & $30.87 \pm 2.23$ & $28.59 \pm 0.46$ & 1.00 & $14.85 \pm 3.34$ & $11.17 \pm 0.18$ & 1.10 & No excess \\
\hline 109176 & 210027 & $34.70 \pm 2.38$ & $34.35 \pm 0.51$ & 0.14 & $16.90 \pm 3.39$ & $13.42 \pm 0.20$ & 1.03 & No excess \\
\hline 113283 & 216803 & $12.21 \pm 1.57$ & $12.48 \pm 0.25$ & -0.17 & $12.69 \pm 1.73$ & $4.87 \pm 0.10$ & 4.51 & Excess \\
\hline 116771 & 222368 & $42.11 \pm 2.66$ & $29.19 \pm 0.44$ & 4.79 & $21.75 \pm 3.33$ & $11.40 \pm 0.17$ & 3.10 & Excess \\
\hline \multicolumn{9}{|c|}{ FGK stars $(15 \mathrm{pc}<d \leq 20 \mathrm{pc})$} \\
\hline 5862 & 7570 & $24.36 \pm 2.03$ & $13.83 \pm 0.14$ & 5.16 & $7.33 \pm 3.29$ & $5.40 \pm 0.06$ & 0.59 & Excess \\
\hline 17651 & 23754 & $26.70 \pm 2.16$ & $21.10 \pm 0.53$ & 2.52 & $8.22 \pm 3.23$ & $8.24 \pm 0.21$ & -0.01 & No excess \\
\hline 36366 & 58946 & $22.24 \pm 1.92$ & $18.79 \pm 0.32$ & 1.77 & $18.78 \pm 3.47$ & $7.34 \pm 0.12$ & 3.30 & Dubious \\
\hline 44248 & 76943 & $34.66 \pm 2.29$ & $28.35 \pm 0.59$ & 2.67 & $22.61 \pm 3.38$ & $11.07 \pm 0.23$ & 3.40 & Dubious \\
\hline 46509 & 81997 & $19.15 \pm 1.91$ & $16.06 \pm 0.13$ & 1.62 & $9.20 \pm 3.36$ & $6.27 \pm 0.05$ & 0.87 & No excess \\
\hline 48113 & 84737 & $9.56 \pm 1.56$ & $13.94 \pm 0.14$ & -2.80 & $9.77 \pm 3.35$ & $5.44 \pm 0.06$ & 1.29 & No excess \\
\hline $61174^{*}$ & 109085 & $252.00 \pm 16.00$ & $16.00 \pm 0.43$ & 14.75 & $231.00 \pm 13.00$ & $6.25 \pm 0.17$ & 17.29 & Excess \\
\hline 64241 & $114378 \mathrm{~J}$ & $26.01 \pm 2.02$ & $21.70 \pm 0.14$ & 2.13 & $16.09 \pm 3.09$ & $8.48 \pm 0.05$ & 2.47 & No excess \\
\hline 67153 & 119756 & $19.70 \pm 1.88$ & $19.51 \pm 0.20$ & 0.10 & $5.72 \pm 2.84$ & $7.62 \pm 0.08$ & -0.67 & No excess \\
\hline 71284 & 128167 & $39.45 \pm 2.57$ & $16.13 \pm 0.09$ & 9.06 & $18.40 \pm 3.13$ & $6.30 \pm 0.04$ & 3.87 & Excess \\
\hline 75312 & 137107J & $12.01 \pm 1.86$ & $14.53 \pm 0.25$ & -1.34 & $4.69 \pm 3.45$ & $5.68 \pm 0.10$ & -0.29 & No excess \\
\hline 77760 & 142373 & $22.41 \pm 1.99$ & $23.38 \pm 0.16$ & -0.49 & $17.09 \pm 3.25$ & $9.13 \pm 0.06$ & 2.45 & No excess \\
\hline $88745^{*}$ & 165908 & $87.00 \pm 10.00$ & $15.69 \pm 0.75$ & 7.11 & $80.00 \pm 15.00$ & $6.13 \pm 0.29$ & 4.92 & Excess \\
\hline 104858 & 202275 & $25.12 \pm 2.00$ & $20.14 \pm 0.19$ & 2.48 & $5.26 \pm 3.61$ & $7.87 \pm 0.07$ & -0.72 & No excess \\
\hline 112447 & 215648 & $26.32 \pm 2.19$ & $27.73 \pm 0.29$ & -0.64 & $16.19 \pm 3.19$ & $10.83 \pm 0.11$ & 1.68 & No excess \\
\hline
\end{tabular}

Notes. ${ }^{(*)}$ denotes that the source is extended. The uncertainties $\sigma\left(\operatorname{PACS}_{v}(\lambda)\right)$ for PACS100 and PACS160 are the statistical plus systematic, combined quadratically; values of $5 \%$ of the fluxes for the systematic uncertainties (calibration) were considered at both wavelengths (Balog et al. 2014). PACS fluxes for HIP 61174 are from Duchêne et al. (2014). PACS fluxes for HIP 88745 are from Kennedy et al. (2012). HIP 7751 has been treated as a single source in the statistical analysis of the sample. HIP 72659 is a binary $(\mathrm{G} 7-8 \mathrm{~V}+\mathrm{K} 5 \mathrm{~V})$; however, the SED is best fitted with the G8 photosphere only. HIP 73695 is a W UMa eclipsing binary (F5V+G9V); however, the SED is best fitted with the G9 photosphere only. S100 and S160 values for HIP 88745 (F7V+K4V) are from a composite model so as to include both components of the binary. PACS70 fluxes for HIP 15510 (100.0 \pm 5.0 mJy, Kennedy et al. 2012), HIP 61174 (230.0 \pm 13.0 mJy, Duchêne et al. 2014), HIP 64924 (198.0 \pm 3.0 mJy, Wyatt et al. 2012), and HIP $88745(93.0 \pm 10.0$ mJy, Kennedy et al. 2012) have also been used in this work. In addition to the PACS data, SPIRE flux densities have been included in the SEDs of these sources: HIP 61174: $100.0 \pm 10.0(250 \mu \mathrm{m}),<100.0(350 \mu \mathrm{m}),<10.0 \mathrm{mJy}(500 \mu \mathrm{m})($ Duchêne et al. 2014). HIP 64294: $129.0 \pm 21.0(250 \mu \mathrm{m}), 55.0 \pm 17.0(350 \mu \mathrm{m}), 20.0 \pm 12.0 \mathrm{mJy}(500 \mu \mathrm{m})$ (Wyatt et al. 2012). HIP $88745: 44.0 \pm 6.0(250 \mu \mathrm{m})$, $22.0 \pm 7.0(350 \mu \mathrm{m})$ (Kennedy et al. 2012). 